\title{
DARK MATTER AND COLOR OCTETS BEYOND THE STANDARD MODEL
}

\author{
by
}

Gordan Z. Krnjaic

\begin{abstract}
A dissertation submitted to The Johns Hopkins University in conformity with the requirements for the degree of Doctor of Philosophy.
\end{abstract}

Baltimore, Maryland

July, 2012 


\section{Abstract}

Although the Standard Model (SM) of particles and interactions has survived forty years of experimental tests, it does not provide a complete description of nature. From cosmological and astrophysical observations, it is now clear that the majority of matter in the universe is not baryonic and interacts very weakly (if at all) via non-gravitational forces. The SM does not provide a dark matter candidate, so new particles must be introduced. Furthermore, recent Tevatron results suggest that SM predictions for benchmark collider observables are in tension with experimental observations. In this thesis, we will propose extensions to the SM that address each of these issues.

Although there is abundant indirect evidence for the existence of dark matter, terrestrial efforts to observe its interactions have yielded conflicting results. We address this situation with a simple model of dark matter that features hydrogen-like bound states that scatter off SM nuclei by undergoing inelastic hyperfine transitions. We explore the available parameter space that results from demanding that DM self-interactions satisfy experimental bounds and ameliorate the tension between positive and null signals at the DAMA and CDMS experiments respectively. 


\section{ABSTRACT}

However, this simple model does not explain the cosmological abundance of dark matter and also encounters a Landau pole at a low energy scale. We, therefore, extend the field content and gauge group of the dark sector to resolve these issues with a renormalizable UV completion. We also explore the galactic dynamics of unbound dark matter and find that "dark ions" settle into a diffuse isothermal halo that differs from that of the bound states. This suppresses the local dark-ion density and expands the model's viable parameter space.

We also consider the $>3 \sigma$ excess in $W$ plus dijet events recently observed at the Tevatron collider. We show that decays of a color-octet, electroweak-triplet scalar particle ("octo-triplet") can yield the requisite final state to explain the data. We also find that octotriplets can induce mixing in the $B-\bar{B}$ system and may give rise to additional $\mathrm{CP}$ violation. The model makes concrete predictions for several final states accessible at the LHC, so it can promptly be discovered or falsified.

Finally we address the anomalous top forward-backward asymmetry observed the Tevatron. We find that a spin-1 color octet particle with flavor blind axial interactions can explain this anomaly if the mass is in the $50-90 \mathrm{GeV}$ range. We explore the multitude of experimental constrains in this mass window and present the viable parameter space as a function of the axigluon mass and coupling constant.

Advisor: David E. Kaplan 


\section{Acknowledgements}

This thesis would not have been possible without the wisdom and encouragement of my advisor David E. Kaplan. His guidance, support, and incredible physical intuition have influenced every aspect of my graduate work and I am tremendously grateful for the interactions we've had. I am also deeply indebted to Bogdan Dobrescu who has been both a great collaborator and a surrogate advisor. I am lucky to have collaborated with Marc Kamionkowski whose insights have expanded my horizons. I am also indebted to my friends and collaborators Christopher Wells and Keith Rehermann who taught me a great deal about physics and other things too. I have benefited tremendously from interactions with Roni Harnik, Graham Kribs, Paddy Fox, Andreas Kronfeld, Kirill Melnikov, Raman Sundrum, Adam Martin, Gerben Stavenga, Ciaran Williams, Joachim Kopp, Johan Alwall, Nausheen Shah, Ethan Neil, Daniel Stolarski, Matt Baumgart, Yuhsin Tsai, Christopher Bouchard, and Alejandro de la Puente. Finally I would like to express my gratitude to the particle theory groups at Johns Hopkins and Fermilab; thanks for giving me a chance to learn the coolest job in the world. 


\section{Dedication}

I dedicate this thesis to my parents Zdenko and Nada whose deep respect for education has molded every aspect of my life. Without their great love, unwavering support, and innumerable sacrifices on my behalf, nothing in my life would have been possible. I owe you everything. 


\section{Contents}

Abstract

Acknowledgements

iv

List of Tables

$\mathbf{x}$

List of Figures

xi

1 Introduction

1.0.1 Standard Model Description . . . . . . . . . . . . . . . . 3

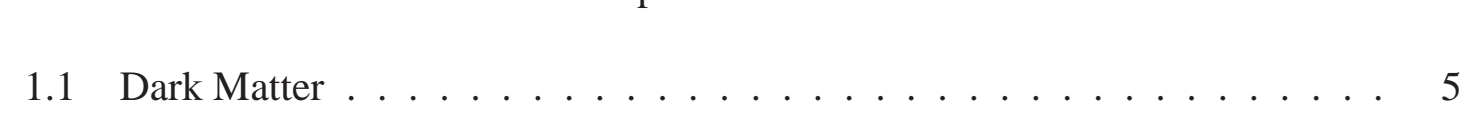

1.1.1 Direct Detection and DAMA $\ldots \ldots \ldots \ldots$

1.1.2 Inelastic Dark Matter . . . . . . . . . . . . . . 7

1.2 Tension at the Tevatron $\ldots \ldots \ldots \ldots \ldots \ldots$

1.2.1 CDF Dijet Excess. . . . . . . . . . . . . . . . . 9

1.2.2 Top Forward-Backward Asymmetry . . . . . . . . . . . . . . 11 


\section{CONTENTS}

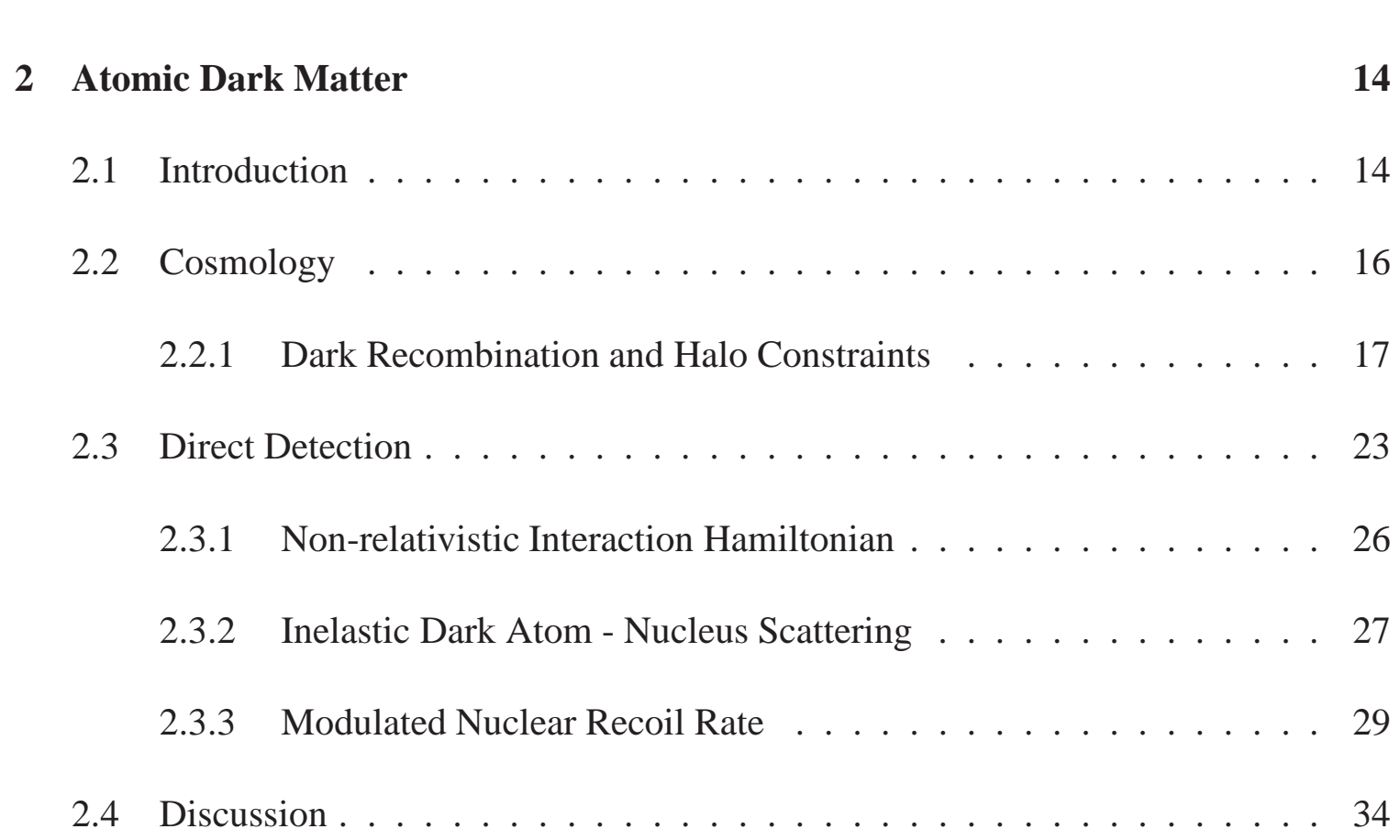

3 UV Completion: Dark Atoms

3.1 Introduction . . . . . . . . . . . . . . . 37

3.2 Review of $\mathrm{aDM} \ldots \ldots \ldots \ldots \ldots$

3.2 .1 Cosmology . . . . . . . . . . . . . . . . . 40

3.2.2 Direct Detection ........................ 41

3.3 Asymmetric Atomic Dark Matter . . . . . . . . . . . . . 42

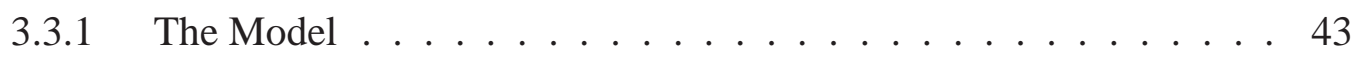

3.3.2 Connecting Atomogenesis to Leptogenesis . . . . . . . . . . . . . 44

3.3.3 Symmetry breaking and IR mass spectrum . . . . . . . . . . . . . 48

3.3.4 Recombination of Multiple Atomic Species . . . . . . . . . . 49

3.3.5 Light Degrees of Freedom and the CMB . . . . . . . . . . . 52

3.4 Direct Detection and Allowed Parameter Space . . . . . . . . . . . . . 53 


\section{CONTENTS}

3.4 .1 Isothermal Ionic Halo . . . . . . . . . . . . . . . . . 53

3.4 .2 Direct Detection . . . . . . . . . . . . . 58

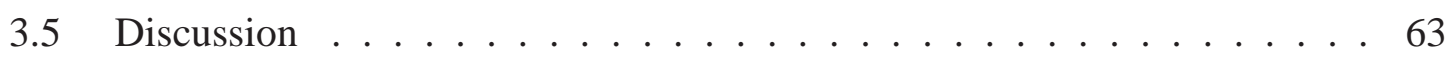

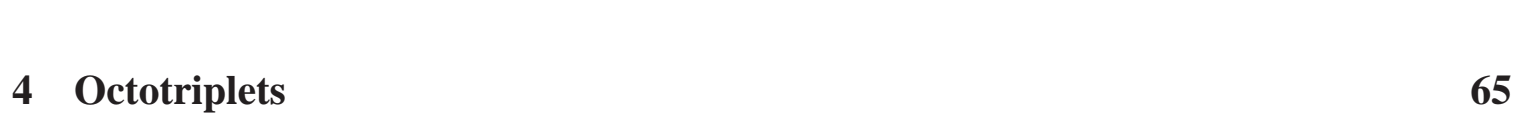

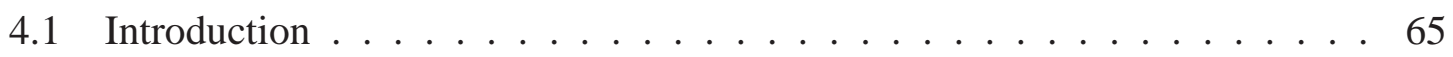

4.2 Octo-triplet scalar $\ldots \ldots \ldots \ldots \ldots$

4.2.1 Interactions and masses . . . . . . . . . . . . . . 68

4.2.2 Collider signals of octo-triplets ... . . . . . . . 71

4.3 Octo-triplet decavs via higher-dimensional operators . . . . . . . . 76

4.3.1 Octo-triplet plus a vectorlike quark . . . . . . . . . . . . 77

$4.3 .2 B_{s}-\bar{B}_{c}$ mixing $\ldots \ldots \ldots . \ldots \ldots 1$

4.3.3 Dijet resonance plus a $W$ boson at the Tevatron . . . . . . . . . . . 84

4.3 .4 Resonant production of $\Theta^{+} \Theta^{-} \ldots \ldots$. . . . . . . . . . 90

4.3 .5 LHC Signals . . . . . . . . . . . . . . . . 95

4.4 Conclusions . . . . . . . . . . . . . . . . . . . . . . . . . . . 97

5 Verv Light Axigluons $\quad 107$

5.1 Introduction . . . . . . . . . . . . . . . 107

5.2 Model Description . . . . . . . . . . . . . . . . 110

5.3 Simulation and Acceptances $\ldots \ldots \ldots$. . . . . . . . . . . . . 112

5.4 Experimental Constraints . . . . . . . . . . . . . . 113 


\section{CONTENTS}

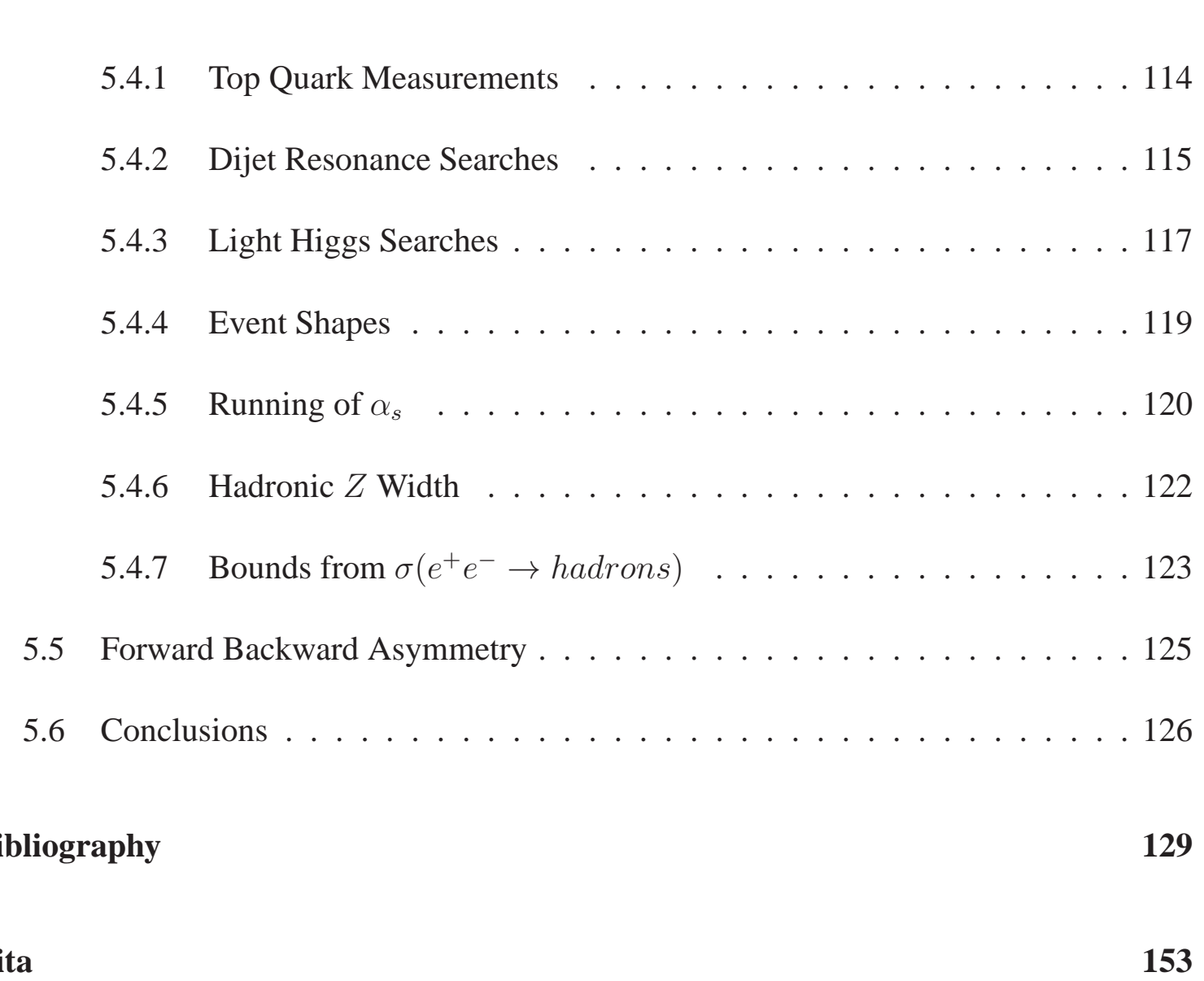




\section{List of Tables}

$1.1 \quad$ Standard Model field content and charge assignments . . . . . . . 3

$3.1 \quad$ Field content and $U(1)$ charges for aDM. . . . . . . . . . 40

$3.2 \quad$ Field content and gauge representations for Asymmetric aDM. . . . 44 


\section{List of Figures}

$2.1 \quad$ The allowed parameter space in $\alpha_{D}-m_{\mathrm{e}} \ldots \ldots \ldots \ldots$

$2.2 \quad$ Examples of modulated spectra at DAMA . . . . . . . . . 30

2.3 The allowed parameter space for $M_{X}$ and $g_{5} \ldots \ldots . \ldots 33$

$3.1 \quad$ Diagrams contributing to scalar doublet decay $\ldots \ldots \ldots$. . . 46

$3.2 \quad$ Plot of species abundance. . . . . . . . . . . . . 51

$3.3 \quad$ Plot of atomic ionized mass densities . . . . . . . . . 57

$3.4 \quad$ DAMA and CoGeNT favored regions . . . . . . . . . 61

$4.1 \quad$ Charged octo-triplet decav to a $W$ boson and a gluon. . . . . . . . 72

$4.2 \quad$ Leading order octo-triplet cross section . . . . . . . . . . . 74

$4.3 \quad$ Octo-triplet decav through vectorlike doublet $\ldots \ldots \ldots$. . . 78

$4.4 \quad$ Leading contribution to $B_{s}-\bar{B}_{s}$ mixing $\ldots \ldots \ldots \ldots$. . . 82

$4.5 \quad$ Pair production of charged octo-triplets through gluon fusion . . . 84

$4.6 \quad$ Partonic $p_{T}$ distributions for final state quarks $\ldots \ldots \ldots$. . . 85

$4.7 \quad$ Exclusive $M_{i j}$ distribution for the leading two iets $\ldots \ldots \ldots 87$

$4.8 \quad$ Inclusive $M_{j j}$ distribution for the leading two jets $\ldots \ldots \ldots$

4.9 Same as Figure 4.5 but including coloron diagrams. . . . . . . . 91

$4.10 \quad$ Same as Figure 4.7 but including coloron diagarams . . . . . . . 92

4.11 Same as Figure 4.10 but inclusive with $2+$ iets passing cuts. . . . . 93

$4.12 \quad p_{T}$ distribution of the dijet system for events passing cuts $\ldots . .99$

$4.13 \quad \Delta R_{j j}$ of the two leading jets $\ldots \ldots \ldots \ldots \ldots$

$4.14 \quad 3$-body decay of the charged octo-triplet . . . . . . . . 102

$5.1 \quad t \bar{t}$ production through an axigluon $\ldots \ldots \ldots \ldots$

$5.2 \quad M_{\bar{t}}$ distribution including axigluon production $\ldots \ldots \ldots 115$

$5.3 \quad$ Axigluon parameter space in the $\left(\lambda . m_{c^{\prime}}\right)$ plane $\ldots \ldots \ldots . .117$

$5.4 \quad$ Inclusive $t \bar{t}$ rapidity difference distribution . . . . . . . . . . . 124

$5.5 \quad$ Theory prediction for the mass dependent $t \bar{t}$ asymmetry . . . . . 127 


\section{Chapter 1}

\section{Introduction}

The Standard Model (SM) of the strong and electroweak interactions is perhaps the crowning achievement of theoretical physics. It realizes the most general, renormalizable quantum field theory invariant under an $S U(3)_{c} \times S U(2)_{L} \times U(1)_{Y}$ symmetry with local gauge invariance. The subscript $c$ refers to the "color" charge associated with strong force, $L$ stands for the "left-handed" chiral nature of the $S U(2)_{L}$ interactions, and $Y$ is the "hypercharge" associated with the abelian subgroup. Every known particle is charged under a representation of these three groups.

The strong force, Quantum Chromodynamics (QCD), is described by the dynamics of $S U(3)_{c}$ interactions involving spin $1 / 2$ quarks that carry one of three possible color charges (red, green, or blue) and spin 1 gluons that carry both color and anti-color charges. The strength of the force between color-charged objects increases with their separation distance, so that on everyday length scales (and temperatures), quarks and gluons are not observed 


\section{CHAPTER 1. THE STANDARD MODEL AND ITS DISCONTENTS}

as free-particles; they form bound states known as hadrons - e.g. protons and neutrons.

The electroweak force is described by the remaining $S U(2)_{L} \times U(1)_{Y}$ gauge symmetry and gives rise to both electromagnetism and the "weak" force, which is responsible for various nuclear processes - e.g. beta decay. The quarks, leptons (spin 1/2 fermions uncharged under QCD - e.g. electrons or neutrinos), and Higgs boson all carry charges under both product groups, however the $S U(2)_{L}$ subgroup is chiral; its gauge bosons only mediate interactions between the left-handed projections of fermion fields. The full $S U(2)_{L} \times U(1)_{Y}$ symmetry is exact at the level of the fundamental equations of the theory, but is "broken" by the low-temperature vacuum, which is only invariant under the Quantum Electrodynamics $(\mathrm{QED})$ subgroup $U(1)_{Q E D}$, whose lone force carrier, the familiar photon, is a linear combination of the electrically neutral gauge bosons from the full electroweak group.

Since its introduction over forty years ago, the SM has withstood a battery of high and low energy tests without any significant discrepancies between its theoretical predictions and and experimental results [1,2]. Nonetheless, the SM is not complete. Based on indirect cosmological and astrophysical evidence, we know that non-baryonic dark matter (DM) comprises $\sim 80 \%$ of the matter in our universe, yet the SM offers no plausible dark matter candidate. Furthermore, in recent years, the Tevatron has seen several hints of new particles or forces beyond the SM. In this thesis, we will present several extensions to the SM that propose new dark matter candidates and attempt to explain the new collider anomalies. We begin with a detailed description of the SM field content. 


\section{CHAPTER 1. THE STANDARD MODEL AND ITS DISCONTENTS}

\subsubsection{Standard Model Description}

The SM features three generations spin $1 / 2$ of quark fields $\left\{Q_{L}=\left(u_{L}, d_{L}\right), u_{R}, d_{R}\right\}$, three generations of spin $1 / 2$ lepton fields $\left\{L=\left(\nu_{L}, e_{L}\right), e_{R}\right\}$, and a spinless Higgs boson $H$ all charged under an $S U(3)_{c} \times S U(2)_{L} \times U(1)_{Y}$ gauge symmetry. The spin 1 gauge bosons $\left\{G_{\mu}^{a}, W_{\mu}^{\alpha}, B_{\mu}\right\}$ charged as adjoints under $S U(3)_{c} \times S U(2)_{L} \times U(1)_{Y}$ respectively are the force carriers that mediate interactions between quark, letpon, and higgs fields (See Table 1.1.

\begin{tabular}{|c|c|c|c|c|}
\hline & $S U(3)_{c}$ & $S U(2)_{L}$ & $U(1)_{Y}$ & Spin \\
\hline$Q_{L}^{i}$ & $\square$ & $\square$ & $+1 / 6$ & $1 / 2$ \\
$u_{R}^{i}$ & $\square$ & 1 & $+2 / 3$ & $1 / 2$ \\
$d_{R}^{i}$ & $\square$ & 1 & $-1 / 3$ & $1 / 2$ \\
$L^{i}$ & 1 & $\square$ & $-1 / 2$ & $1 / 2$ \\
$e_{R}^{i}$ & 1 & 1 & -1 & $1 / 2$ \\
\hline \hline$G_{\mu}^{a}$ & Adj. & 1 & 0 & 1 \\
$W_{\mu}^{\alpha}$ & 1 & $\operatorname{Adj}$. & 0 & 1 \\
$B_{\mu}$ & 1 & 1 & 0 & 1 \\
\hline \hline$H^{i}$ & 1 & $\square$ & $+1 / 2$ & 0 \\
\hline
\end{tabular}

Table 1.1: Standard Model field content and $S U(3)_{c} \times S U(2)_{L} \times U(1)_{Y}$ charge assignments. For each fermionic field the index $i=1,2,3$ labels the generation.

All SM interactions are given by the most general renormalizable lagrangian invariant 


\section{CHAPTER 1. THE STANDARD MODEL AND ITS DISCONTENTS}

under all gauge symmetries

$$
\begin{aligned}
\mathcal{L}_{S M}= & -\frac{1}{4} B^{\mu \nu} B_{\mu \nu}-\frac{1}{4} W^{\alpha \mu \nu} W^{\alpha}{ }_{\mu \nu}-\frac{1}{4} G^{a \mu \nu} G_{\mu \nu}^{a}+\sum_{\psi} \bar{\psi} i \not D \psi+\left|D_{\mu} H\right|^{2}+\mu^{2}|H|^{2} \\
& -\lambda|H|^{4}-\sum_{i j}\left(y_{i j}^{u} \bar{Q}_{L}^{i} \tilde{H} u_{R}^{j}+y_{i j}^{d} \bar{Q}_{L}^{i} H d_{R}^{j}+y_{i j}^{e} \bar{E}^{i} H e_{R}^{j}+H . c .\right)
\end{aligned}
$$

where $\tilde{H} \equiv i \sigma^{2} H^{\dagger}, \sigma^{2}$ is the second Pauli matrix, $a=1, . ., 8$ is an $S U(3)_{c}$ color index, $\alpha=1,2,3$ is an $S U(2)_{L}$ electroweak index, $i=1,2,3$ is a flavor index, $\psi$ is any of the fermion fields in Table 1.1, $\mu$ is the Higgs mass parameter, $\lambda$ is the dimensionless Higgs quartic coupling, and $y_{i j}^{u, d, e}$ are dimensionless Yukawa couplings. The gauge field-strength tensors are

$$
\begin{aligned}
B_{\mu \nu} & \equiv \partial_{\mu} B_{\nu}-\partial_{\nu} B_{\mu} \\
W_{\mu \nu}^{\alpha} & \equiv \partial_{\mu} W_{\nu}^{\alpha}-\partial_{\nu} W_{\mu}^{\alpha}+g \epsilon^{\alpha \beta \gamma} W_{\beta}^{b} W_{\nu}^{\gamma} \\
G_{\mu \nu}^{a} & \equiv \partial_{\mu} G_{\nu}^{a}-\partial_{\nu} G_{\mu}^{a}+g_{s} f^{a b c} G_{\mu}^{b} G_{\nu}^{c}
\end{aligned}
$$

where $g$ and $g_{s}$ are the $S U(2)_{L}$ and $S U(3)_{c}$ coupling constants and $\epsilon^{\alpha \beta \gamma}$ and $f^{a b c}$ are the corresponding group structure constants. The most general covariant derivative acting on any field that transforms under representations $G_{3}$ and $G_{2}$ of $S U(3)_{c} \times S U(2)_{L}$ respectively and carries $U(1)_{Y}$ hypercharge of $Y$ (see column 3 in Table 1.1) is given by

$$
D_{\mu} \equiv \partial_{\mu}-i g_{s}\left(T_{G_{3}}^{a}\right) G_{\mu}^{a}-i g\left(T_{G_{2}}^{\alpha}\right) W_{\mu}^{\alpha}-i g^{\prime} Y B_{\mu}
$$

where $g^{\prime}$ is the coupling constant for $U(1)_{Y}$, and $T_{G_{3}}^{a}, T_{G_{2}}^{\alpha}$, are generator matrices for representations $G_{3}$ and $G_{2}$ of $S U(3)_{c}$ and $S U(2)_{L}$ respectively.

Upon the breakdown of electroweak symmetry $S U(2)_{L} \times U(1)_{Y} \rightarrow U(1)_{Q E D}$, the Higgs scalar acquires a vacuum expectation value in its neutral component $\langle H\rangle=$ 


\section{CHAPTER 1. THE STANDARD MODEL AND ITS DISCONTENTS}

$(0 v / \sqrt{2})^{T}$, where $v=246 \mathrm{GeV}$, and gives masses to linear combinations of $W_{\mu}^{\alpha}$ and $B_{\mu}$ fields. In the mass eigenbasis we can define the $W^{ \pm}$and $Z$ bosons as

$$
W_{\mu}^{ \pm} \equiv \frac{1}{\sqrt{2}}\left(W_{\mu}^{1} \mp i W_{\mu}^{2}\right) \quad, \quad Z_{\mu} \equiv \frac{g W_{\mu}^{3}-g^{\prime} B_{\mu}}{\sqrt{g^{2}+g^{\prime 2}}}
$$

with corresponding masses $m_{W}=g v / 2$ and $m_{Z}=\sqrt{g^{2}+g^{\prime 2}} v / 2$. The other linear combination of electrically neutral gauge bosons becomes the familiar photon of Quantum Electrodynamics (QED)

$$
A_{\mu} \equiv \frac{g^{\prime} W_{\mu}^{3}+g B_{\mu}}{\sqrt{g^{2}+g^{\prime 2}}}
$$

which remains massless.

\subsection{Dark Matter}

There is tremendous indirect evidence for the existence of dark matter, which comprises roughly $80 \%$ of the matter in our universe. The presence of dark matter can be inferred from the CMB power spectrum [3], surveys of large scale structure [4], galactic rotation curves [5-7], and weak lensing observations [8]. Although this evidence does not require DM to participate in non-gravitational interactions, many of the best motivated SM extensions (e.g. supersymmetry, extra dimensions) feature plausible dark matter candidates that interact weakly with visible particles. This possibility motivates direct detection efforts to observe DM collisions off SM nuclei in terrestrial detectors. 


\section{CHAPTER 1. THE STANDARD MODEL AND ITS DISCONTENTS}

\subsubsection{Direct Detection and DAMA}

Direct detection experiments typically involve underground detectors designed to observe the recoil of a target nucleus after a collision with a dark matter particle as it passes through the Earth. The rate per unit recoil energy is proportional to the number of target nuclei and the flux of incoming dark matter particles:

$$
\frac{d R}{d E_{R}}=N_{T} n_{D M} \int_{v_{\min }\left(E_{R}\right)}^{v_{e s c}} f(v) \frac{d \sigma}{d E_{R}} v d v
$$

where $N_{T}$ is the number of target nuclei, $n_{D M}$ is the local number density of dark matter particles, $\sigma$ is the DM-nucleus scattering cross section, $v$ is the DM speed relative to the target, $f(v)$ is the distribution of DM speeds in the laboratory reference frame, and $v_{e s c}$ is the galactic escape velocity. For elastic scattering, the minimum speed $v_{\min }$ that a DM particle must have to induce a nuclear recoil of energy $E_{R}$ is

$$
v_{\text {min }}\left(E_{R}\right)=\frac{1}{\mu} \sqrt{\frac{m_{N} E_{R}}{2}} \quad, \quad \mu=\frac{m_{N} m_{D M}}{m_{N}+m_{D M}}
$$

where $m_{N}$ is the nuclear target mass, $m_{D M}$ is the dark matter mass and $\mu$ is the reduced mass of the system. Importantly, there is no lower threshold for recoil energies; DM particles of any speed can produce a recoil, so for a typical Maxwellian $f(v)$, the recoils are due predominantly to DM from the peak of the distribution.

The dark matter halo is stationary in the galactic rest frame, but the Sun has a constant velocity through the galaxy, so as the earth orbits the Sun, the relative velocity between the Earth and the DM halo depends on the alignment of Earth and Sun velocities. This motion makes $f(v)$ time-dependent and periodic in the Earth frame, shifting towards higher 


\section{CHAPTER 1. THE STANDARD MODEL AND ITS DISCONTENTS}

velocities when Earth and Sun velocities are parallel (June $2^{\text {nd }}$ ) and towards lower velocities when they are anti-parallel (Dec. $2^{\text {nd }}$ ). Since all the time dependence in direct detection comes from $f(v)$, any DM/SM interaction yields an anually modulated event rate. The DAMA collaboration claims to have detected this modulation, with the correct period, and with a statistical significance of $8.2 \sigma$ [9]. However, when the null results of other direct DM searches are interpreted as constraints on elastic DM scattering, all of the parameter space preferred by DAMA's signal is ruled out (See for example Ref. [10].)

\subsubsection{Inelastic Dark Matter}

If we abandon the assumption of elastic scattering, it is possible to reconcile DAMA with other null results [11]. In the Inelastic Dark Matter (iDM) framework, the dark sector contains two species of particle whose masses differ by some small amount $\delta$ and, upon scattering, the lighter species converts into the heavier one. The mass splitting gives rise to a threshold recoil energy - and DM speed - not present in the elastic case

$$
v_{\text {min }}\left(E_{R}\right)=\frac{1}{\sqrt{2 m_{N} E_{R}}}\left(\frac{m_{N} E_{R}}{\mu}+\delta\right) \quad, \quad v_{\text {threshold }}=\sqrt{\frac{2 \delta}{\mu}}
$$

Unlike with the elastic case in Eq. (1.1.9), here $E_{R}$ cannot be arbitrarily small and there is always a threshold velocity below which all scattering is kinematically forbidden. Depending on the size of $\delta$, this threshold can also lead to substantially higher annual modulation than in the elastic case. It is even possible to choose $\delta$ so that the scattering rate vanishes when the velocity distribution shifts towards lower velocities later in the year. This 


\section{CHAPTER 1. THE STANDARD MODEL AND ITS DISCONTENTS}

effect weakens the constraints from direct detection experiments whose data were gathered primarily during wintertime in the northern hemisphere. Note also the dependence of Eq. (1.1.10) on the nuclear mass: $v_{\text {threshold }}$ grows as $m_{N}$ decreases, so inelastic scattering favors heavier targets. The DAMA detector consists of Iodine nuclei, so the constraints from experiments using lighter nuclei (e.g. Xenon and Germanium) are also weakened.

With a dark matter mass $m_{D M} \sim 100 \mathrm{GeV}$ and an inelasticity $\delta \sim 100 \mathrm{keV}$ the combination of the effects above can explain the DAMA results and simultaneously evade bounds from other direct detection experiments. The apparent drawback to this approach is that it seems highly unnatural for such a small mass splitting to arise in a theory whose typical mass scale is one million times bigger. However, in composite quantum systems, like ordinary hydrogen atoms, hierarchical energy splittings of this sort arise quite naturally. In Chapter 2, motivated by this feature, we will explore the possibility that DM exists primarily in the form of atomic bound states that scatter off SM nuclei by undergoing inelastic hyperfine transitions. In Chapter 3 we will revisit atomic dark matter and present an ultraviolet (UV) completion that gives rise to the same low energy atomic bound states, but also generates the observed DM abundance in the early universe and tames the non-perturbative high-energy behavior of the original simple model. 


\section{CHAPTER 1. THE STANDARD MODEL AND ITS DISCONTENTS}

\subsection{Tension at the Tevatron}

As the LHC begins to explore the high energy frontier, there are already some tantalizing hints of discrepancies in the SM framework. In its final days, experiments at the Tevatron proton anti-proton collider reported several hints of physics beyond the SM. In this thesis we will consider models to address two of these: the $W$ plus dijet excess observed by the CDF collaboration and the large forward-backward asymmetry in top anti-top production seen by both $\mathrm{CDF}$ and $D \emptyset$. Although these anomalies have not yet reached the $5 \sigma$ of statistical significance traditionally required to declare discoveries of new phenomena,

\subsubsection{CDF Dijet Excess}

A crucial test of the the SM's nonabelian character involves the three gauge-boson interactions in Eq. (1.0.1). In the mass eigenbasis there are $W^{+} W^{-} Z$ and $W^{+} W^{-} A$ operators, which have no analogues in purely abelian theories. At the Tevatron, these interactions can be probed in proton anti-proton collisions which produce gauge boson pairs

$$
\begin{aligned}
& p \bar{p} \rightarrow W^{ \pm} Z \rightarrow \ell^{ \pm} \nu q \bar{q} \rightarrow \ell^{ \pm} \nu j j \\
& p \bar{p} \rightarrow W^{+} W^{-} \rightarrow \ell^{ \pm} \nu q \bar{q} \rightarrow \ell^{ \pm} \nu j j
\end{aligned}
$$

In each process a $W$ decays to a charged lepton and neutrino while the other $W$ or $Z$ decays to two quarks, which evolve into "jets" $(j)$ of hadronic matter. 


\section{CHAPTER 1. THE STANDARD MODEL AND ITS DISCONTENTS}

A typical experimental probe of such events is the two-jet invariant mass distribution where the invariant mass $m_{j j}$ is defined as

$$
m_{j j} \equiv \sqrt{\left(E_{1}+E_{2}\right)^{2}-\left(\vec{p}_{1}+\vec{p}_{2}\right)^{2}}
$$

Here $E_{1,2}$ and $\vec{p}_{1,2}$ are the energies and three-momenta of the two most energetic jets in an event. Since this quantity is a relativistic invariant, a particle that decays to two quarks, always yields two jets whose $m_{j j}$ is equal to the mother particle's rest mass. By studying this distribution near $m_{W}$ and $m_{Z}$ it is possible to test the accuracy of the SM prediction.

For events identified to have the $\ell \nu j j$ final state, the CDF collaboration [12] observes a large, statistically significant excess in the $m_{j j}$ distribution near $150 \mathrm{GeV}$. This result exceeds the SM prediction by over 4 standard deviations and may be evidence of a new particle that is produced in association with a $W$ boson and decays to a pair of strongly interacting particles.

Since the D $\emptyset$ collaboration does not see a comparable excess [13] and the Tevatron director's task force has been unable to resolve the disagreement, this discrepancy will likely persist until LHC results exhaustively probe the same process. Nonetheless, in Chapter 4 we will show that the $\mathrm{CDF}$ result can be explained with a new particle that transforms as a color octet and electroweak triplet - an "octo-triplet". 


\section{CHAPTER 1. THE STANDARD MODEL AND ITS DISCONTENTS}

\subsubsection{Top Forward-Backward Asymmetry}

Since the top quark is the most massive and least studied SM fermion, it invites a great deal of intrigue. In many well motivated extensions of the SM, it plays a key role in electroweak symmetry breaking [14], so its detailed properties may deviate from naive expectations based on the lagrangian in Eq. (1.0.1). This tantalizing possibility motivates precision measurements of various top quark kinematic properties.

At the Tevatron the top quark is most commonly produced in association with an antitop quark, so it is natural to define forward-backward asymmetry $A_{F B}$ defined as

$$
A_{F B} \equiv \frac{\sigma_{F}-\sigma_{B}}{\sigma_{F}+\sigma_{B}}
$$

where the forward and backward cross sections are

$$
\sigma_{F}=\int_{0}^{1} \frac{d \sigma}{d \cos \theta} d \cos \theta \quad, \quad \sigma_{B}=\int_{-1}^{0} \frac{d \sigma}{d \cos \theta} d \cos \theta
$$

$\sigma$ is the $t \bar{t}$ production cross section, and $\theta$ is the scattering angle in the lab frame. At tree level, all gluon exchange diagrams that produce $t \bar{t}$ pairs are symmetric with respect to the forward and backward directions, so no asymmetry arises. At loop level, however, the SM does predict a small asymmetry $-A_{F B}(S M) \sim 5 \%$ - that arises from interference between tree and loop level diagrams. This sensitivity to quantum effects makes $A_{F B}$ particularly sensitive to new intermediate states that may be kinematically inaccessible at a collider, but still influence the angular distributions of final state particles.

Both Tevatron collaborations have recently reported measurements of $A_{F B}$ in $t \bar{t}$ production with intriguing deviations from SM predictions. CDF's main result [15] stud- 


\section{CHAPTER 1. THE STANDARD MODEL AND ITS DISCONTENTS}

ies the lepton plus jets channel where one of the top quarks decays semi-leptonically: $p \bar{p} \rightarrow t \bar{t} \rightarrow(\ell \nu b)(q \bar{q})$. They report an inclusive parton level asymmetry

$$
A_{F B}(C D F)_{\ell j}=(15.8 \pm 7.4) \%
$$

If their measurement in the dilepton channel $p \bar{p} \rightarrow t \bar{t} \rightarrow(\ell \nu b)(\ell \nu b)$ [16] is combined with this result, the asymmetry becomes

$$
A_{F B}(C D F)_{\ell \ell+\ell j}=(20.9 \pm 6.6) \%
$$

and exceeds the SM prediction [17]- [19] by more than 2 standard deviations.

D0 performs a similar search [20] in the lepton plus jets channel and reports an inclusive parton-level asymmetry

$$
A_{F B}(D 0)_{\ell j}=(19.6 \pm 6.5) \%
$$

which is also more than $2 \sigma$ above the SM prediction. Taken together, these consistent deviations may be evidence for new physics in top quark production.

While all the inclusive measurements are consistent, the CDF lepton plus jets result sees sharp mass dependence [15] in the binned asymmetry

$$
\begin{aligned}
& A_{F B}\left(M_{t \bar{t}}<450 \mathrm{GeV}\right)=(-11.6 \pm 14.6) \%, \\
& A_{F B}\left(M_{t \bar{t}}>450 \mathrm{GeV}\right)=(47.5 \pm 11.4) \%
\end{aligned}
$$

where $M_{t \bar{t}}$ is the invariant mass of the $t \bar{t}$ system. Here the high mass bin is $3.4 \sigma$ above the SM prediction. Although neither D0 nor the complementary CDF dilepton search see the 


\section{CHAPTER 1. THE STANDARD MODEL AND ITS DISCONTENTS}

same effect, both find consistently positive $>2 \sigma$ deviations from the SM over the full $M_{t \bar{t}}$

range. In Chapter 5, we will see that this anomaly can be explained with a very light $(\sim 50$

- $90 \mathrm{GeV}$ ) spin-1, color octet particle with flavor-universal axial couplings to SM quarks. 


\section{Chapter 2}

\section{Atomic Dark Matter}

\subsection{Introduction}

Cosmological observations suggest that dark matter comprises more than $80 \%$ of the matter in the universe $[4,21]$. Much of the effort to explain the origin of dark matter has focused on minimal solutions in which dark matter consists of a single particle species, the most popular being the neutralino in variants of the supersymmetric standard model. Such dark matter models include the compelling feature that weak-scale physics - weakscale mass and weak-force coupling strength - can naturally generate dark matter with the correct cosmological abundance. Dark matter in this broad class is described as weakly interacting massive particles (WIMPs).

However, conflicts do exist between WIMP models and observational data. The direct detection experiment, DAMA [9], sees a positive signal with great significance $(8 \sigma)$, 


\section{CHAPTER 2. ATOMIC DARK MATTER}

yet when interpreted as a standard WIMP, other experiments such as CDMS [24] and XENON10 [25], completely rule out the same parameter space. Also, measured cosmic ray spectra may suggest a new primary source for electrons and positrons in our galaxy and potentially evidence for dark-matter annihilation; however, the standard neutralino candidate is unable to fit this data [26-29].

These issues suggests compelling reasons to explore dark matter models beyond the minimal candidate. In addition, the dark matter sector (or 'dark sector') may be rich with complexity and may feature unanticipated dynamics. In fact, the dark matter may even interact via a long-range force - a massless gauge boson - which is still allowed by the bounds on the number of relativistic degrees of freedom during big bang nucleosynthesis [30].

In this chapter we propose a dark sector charged under a hidden U(1) gauge symmetry. We assume two species of fermions, a 'dark proton' and a 'dark electron', and that the dark matter abundance comes from a matter-anti-matter asymmetry 11 We shall see that in interesting parts of parameter space, the bulk of the dark matter exists in atomic bound states. The Lagrangian is

$$
\mathcal{L}_{\text {dark }}=\bar{\Psi}_{\mathbf{p}}\left(\not D+m_{\mathbf{p}}\right) \Psi_{\mathbf{p}}+\bar{\Psi}_{\mathbf{e}}\left(\not D+m_{\mathbf{e}}\right) \Psi_{\mathbf{e}}
$$

where $\not D=i \not \partial+g Q \not A$ and $Q= \pm 1$ for $\Psi_{\mathbf{p}}$ and $\Psi_{\mathbf{e}}$ respectively. In what follows we use the convention $m_{\mathbf{p}} \geq m_{\mathbf{e}}$ without loss of generality. We show (Section 2.2) that for parts of parameter space, recombination in the dark sector occurs efficiently, and we discuss

\footnotetext{
${ }^{1}$ Some models that use the matter-anti-matter asymmetry to generate the correct dark matter abundance exist $[88,90]$, but we do not explore them here.
} 


\section{CHAPTER 2. ATOMIC DARK MATTER}

the bounds from and implications for structure formation. We then add interactions which allow for direct detection in a way that mimics inelastic dark matter [11] and show that there exist parts of parameter space which can explain the DAMA signal, while avoiding constraints from other direct detection experiments (Section 2.3). Finally, in Section 3.5 we discuss, in a cursory way, other phenomena potentially related to atomic dark matter.

A number of ideas related to this work have appeared in the literature. For example, the idea of U(1) charged dark matter has appeared in [33-36] and the idea of composite dark matter in [37]. To our knowledge, this is the first work to explore the generic parameter space for viable atomic dark matter.

\subsection{Cosmology}

Introducing a new hidden $\mathrm{U}(1)$ has interesting cosmological implications. Our interests lie in the parameter space that affords atomic systems. The existence of standard model (SM) atomic hydrogen states in the early universe requires an asymmetry between particles and antiparticles; dark atoms are no different. We assume that there is a 'dark asymmetry' akin to the baryon asymmetry in the SM, and that the dark asymmetry is such that the

universe is net charge neutral, $n_{\mathbf{e}}=n_{\mathbf{p}} 2_{3}$ The existence of dark atoms implies that dark matter is coupled to dark radiation until the universe cools beyond the binding energy of

\footnotetext{
${ }^{2}$ Unless otherwise noted, e, p, and $\mathbf{H}$ refer to the dark electron, dark proton, and dark hydrogen, respectively.
} 


\section{CHAPTER 2. ATOMIC DARK MATTER}

hydrogen

$$
B=\frac{1}{2} \alpha_{D}^{2} \mu_{\mathbf{H}}
$$

where $\alpha_{D}$ is the dark fine structure constant and $\mu_{\mathbf{H}}=\left(m_{\mathbf{e}} m_{\mathbf{p}}\right) /\left(m_{\mathbf{e}}+m_{\mathbf{p}}\right)$ is the reduced mass of dark hydrogen. This has potentially interesting implications for structure formation because interactions in the dark sector can decouple much later than in a conventional CDM WIMP model. Observations of satellite galaxies seem to favor some mechanism to damp the growth of small scale structure in dark matter [40,41], which, as discussed below, can be provided by atomic dark matter.

\subsubsection{Dark Recombination and Halo Constraints}

One of the most interesting features of the model is the presence of both neutral and ionized dark matter components. The fractional ionization, $X_{\mathbf{e}}$, plays an important role in the cosmic evolution of the dark matter. At early times, $X_{\mathbf{e}}$ affects the decoupling temperature of dark matter and dark radiation, which impacts small-scale structure formation of dark matter. At late times, bounds on dark matter self-interactions constrain $X_{\mathbf{e}}$ because the dark matter ions interact through a long range force. The residual ionization fraction in the dark sector is governed by neutral atom formation in analogy with SM hydrogen recombination [42]. In the following, we follow the notation of Ref. [43].

The residual ionization fraction is found by solving the Boltzmann equation for the free 


\section{CHAPTER 2. ATOMIC DARK MATTER}

dark electron fraction,

$$
X_{\mathbf{e}} \equiv \frac{n_{\mathbf{e}}}{n_{\mathbf{e}}+n_{\mathbf{H}}}
$$

The evolution of $X_{\mathbf{e}}$ depends on the Hubble rate, $\mathrm{H}$, and the rate for $\mathbf{e}+\mathbf{p} \leftrightarrow \mathbf{H}+\gamma$. We can write the thermally-averaged recombination cross section using the dimensionless variable $x=\frac{B}{T}$ as

$$
\langle\sigma v\rangle=\xi \frac{64 \pi}{\sqrt{27 \pi}} \frac{\alpha_{D}^{2}}{\mu_{\mathbf{H}}^{2}} x^{1 / 2} \ln (x)
$$

where $\xi=0.448$ is a best-fit numerical coefficient $[44,45]$. The equation governing $X_{\mathbf{e}}$ can be written as

$$
\frac{d X_{\mathbf{e}}}{d x}=C \frac{1}{H x}\left[\left(1-X_{\mathbf{e}}\right) \beta-X_{\mathbf{e}}^{2} n_{D M}\langle\sigma v\rangle\right]
$$

where

$$
\beta=\langle\sigma v\rangle\left(\frac{B m_{\mathbf{e}}}{2 \pi x}\right)^{3 / 2} \mathrm{e}^{-x}
$$

As discussed in $[42,44]$, recombination into the $n=2$ state completely dominates the evolution of $X_{\mathbf{e}}$. This is accounted for through the factor $C$ in Eq. (2.2.5) which represents the fraction of $n=2$ states that produce a net gain in the number of ground state hydrogen atoms. This is not unity because the thermal bath can ionize the $n=2$ state before it decays. Thus, $C$ is the ratio of the $(n=2 \rightarrow n=1)$ decay rate to the sum of this decay rate plus the ionization rate (see [42] for a detailed discussion)

$$
C=\frac{\Lambda_{\alpha}+\Lambda_{2 \gamma}}{\Lambda_{\alpha}+\Lambda_{2 \gamma}+\beta^{(2)}}
$$




\section{CHAPTER 2. ATOMIC DARK MATTER}
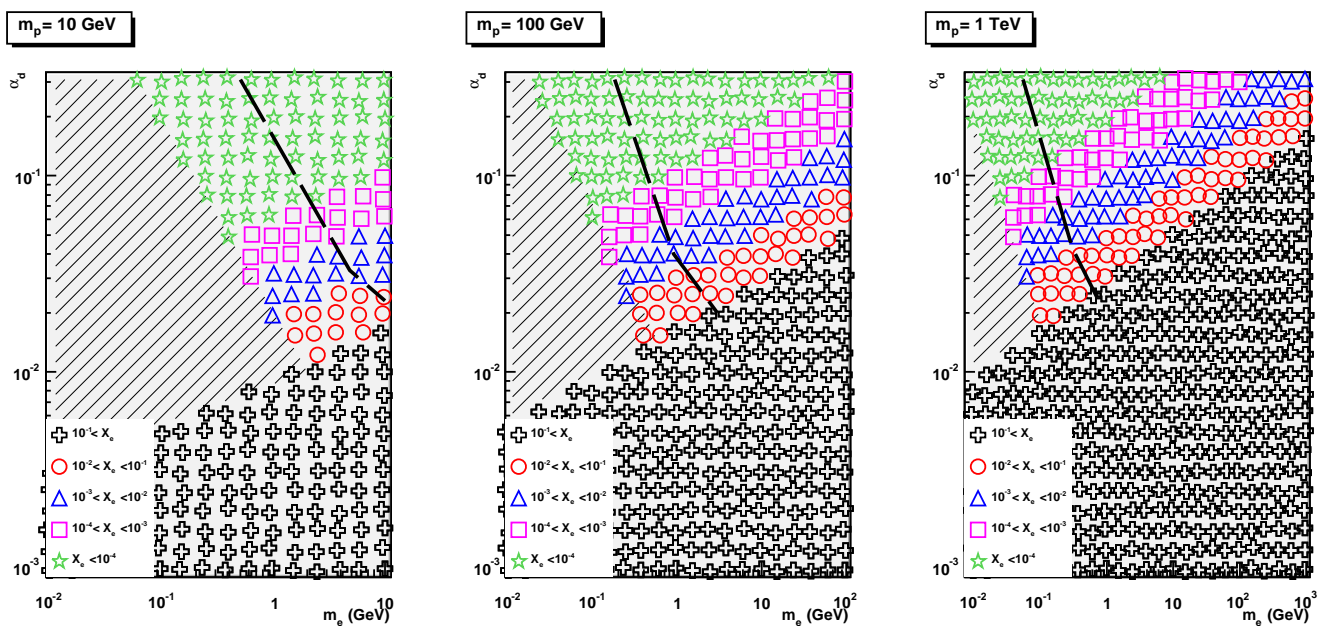

Figure 2.1: The allowed parameter space in $\alpha_{D}-m_{\mathbf{e}}$ for a given $m_{\mathbf{p}}$ and as a function of the residual ionization fraction, $X_{\mathbf{e}}$. Atom dark matter is viable in the colored regions, which correspond to $10^{-2}<X_{\mathbf{e}}<10^{-1}$ (red circles), $10^{-3}<X_{\mathbf{e}}<10^{-2}$ (blue triangles), $10^{-4}<X_{\mathbf{e}}<10^{-3}$ (magenta boxes) and $X_{\mathbf{e}}<10^{-4}$ (green stars). The striped region is ruled out by Eq.2.2.21 with $\kappa=1$ and this region extends to the dashed black line for $\kappa=3$. The black-crossed region is ruled out because $X_{\mathbf{e}}>10 \%$.

The rates are given by

$$
\begin{aligned}
\beta^{(2)} & =\beta \mathrm{e}^{3 x / 4} \\
\Lambda_{\alpha} & =H \frac{(3 B)^{3}}{(8 \pi)^{2}} \frac{1}{\left(1-X_{\mathbf{e}}\right) n_{D M}} \\
\Lambda_{2 \gamma} & =3.8 \frac{3^{2}}{2^{11}} \frac{\alpha^{8} \mu_{H}}{4 \pi}
\end{aligned}
$$

where $\Lambda_{\alpha}$ the rate for a Lyman- $\alpha$ photon to redshift such that it cannot excite $n=1 \rightarrow n=$ 2 and $\Lambda_{2 \gamma}$ is the two photon decay rate of $2 s \rightarrow 1 s$, which has been taken from Ref. [46]. We find that $X_{e}$ varies from $1-10^{-10}$ throughout the parameter space $\alpha_{D} \in\left[10^{-3}, 0.3\right]$ , $m_{\mathbf{e}} \in\left[0.01 \mathrm{GeV}, m_{\mathbf{p}}\right], m_{\mathbf{p}} \in\left[m_{\mathbf{e}}, 3 \mathrm{TeV}\right]$. Self-interactions, as discussed below, rule out some of this parameter space. A few representive planes are plotted in Figure 3.2 


\section{CHAPTER 2. ATOMIC DARK MATTER}

Bounds on the atomic parameter space and $X_{e}$ can be derived from observations of the Bullet Cluster and halo profiles. The bounds are derived through the momentum transfer cross section 3

$$
\sigma_{m t}^{i}=\int d \Omega(1-\cos \theta) \frac{d \sigma^{i}}{d \Omega}
$$

where the index $i$ runs over the three types of self-interactions present in our dark sector: Hydrogen-Hydrogen, Ion-Hydrogen, and Ion-Ion. The last process is described by Coulomb scattering, but since we want to study a dominantly atomic dark sector, the IonIon cross section is the least relevant to our model and we do not discuss it further. Naïvely, the first two cross sections are bounded by geometric values

$$
\frac{d \sigma}{d \Omega} \leq a_{0}^{2}
$$

where $a_{0}=1 /\left(\alpha \mu_{\mathbf{H}}\right)$ is the Bohr radius of dark hydrogen. However, this naïve guess is inadequate. At low energies $\left(k a_{0} \ll 1\right)$ both of these processes can be described by scattering from a central potential

$$
V \propto r^{-n}
$$

with $n=6$ and 4 , respectively. In this case one finds that the cross sections are velocityindependent constants and enhanced over the geometric estimate [47]. We are generally interested in a wide range of $k a_{0}$, thus these results are not strictly applicable however these cross sections are slowly decreasing functions of the relative velocity [47]. A conservative

\footnotetext{
${ }^{3}$ Note that $\sigma_{m t}$ reduces to the total elastic cross section for hard-sphere s-wave scattering which is typical of WIMP dark matter models.
} 


\section{CHAPTER 2. ATOMIC DARK MATTER}

estimate of the cross sections is given by

$$
\frac{d \sigma}{d \Omega} \leq\left(\kappa a_{0}\right)^{2}
$$

with $3 \leq \kappa \leq 10$. The values of $\kappa$ have been inferred from general quantum mechanical scattering $[48,49]$ and detailed computations of SM hydrogen scattering [50-52].

With the relevant cross sections in hand, we can use observations of the Bullet Cluster $[53,54]$ as a guide for the present day maximum value of $X_{\mathbf{e}}$. Measurements of the massto-light ratio and the radius of the sub-cluster suggest that the sub-cluster could have lost no more than $F_{\text {obs }}=20-30 \%$ of its initial mass. Following the analysis in [53], the number of scattering centers that a single dark matter particle encounters as it passes through the target cluster in the case of one species is

$$
\tau=\frac{\sigma}{m} \Xi_{s}
$$

this quantity is often referred to as the scattering depth. The parameter $\Xi_{s}$ is the surface mass density of the sub-cluster defined as

$$
\Xi_{s} \equiv \int_{0}^{R} \rho(z) d z
$$

where $\rho(z)$ is the sub-cluster's volume mass density and $R$ is the radius of the sub-cluster. For multiple species, with species $i$ in the sub-cluster having a mass density $\Xi_{i}$, and mass $m_{i}$, scattering off of species $j$ in the target cluster, we have

$$
\tau_{i j}=\left(\frac{\Xi}{m}\right)_{i} \sigma_{i j} f_{j} \quad(\text { no sum })
$$




\section{CHAPTER 2. ATOMIC DARK MATTER}

where $\sigma_{i j}$ is the cross section for $i$ and $j$ to interact and $f_{j}$ is the number fraction of species $j$ in the target cluster. Equation 2.2.17 can be rewritten in terms the of total observed surface mass density, $\Xi_{T}=\sum_{i} \Xi_{i}$, as

$$
\tau_{i j}=\Xi_{T} \frac{f_{i}}{\sum_{k} f_{k} m_{k}} \sigma_{i j} f_{j}
$$

We make the simplifying and conservative assumption that all of the ions in the subcluster are scattered out of the sub-cluster. In this case, the mass fraction lost through $H-j$ scattering is bounded by

$$
F_{\mathbf{H}}=F_{o b s}-f_{\mathbf{p}} \frac{m_{\mathbf{p}}}{m_{\mathbf{H}}}-f_{\mathbf{e}} \frac{m_{\mathbf{e}}}{m_{\mathbf{H}}}=F_{o b s}-X_{\mathbf{e}}
$$

The mass fraction actually lost from the sub-cluster, using cross sections as parameterized in (2.2.14) and (2.2.18), is

$$
\Delta=\sum_{j} \tau_{H j}=\frac{\Xi_{T}}{m_{\mathbf{H}}}\left(1-X_{\mathbf{e}}^{2}\right) 4 \pi \kappa^{2} a_{0}^{2} .
$$

Demanding that $\Delta<F_{\mathbf{H}}$ we have the bound

$$
\frac{\sigma}{m_{\mathbf{H}}}=\frac{4 \pi \kappa^{2} a_{0}^{2}}{m_{\mathbf{H}}}<\frac{F_{o b s}-X_{\mathbf{e}}}{\Xi_{T}\left(1-X_{\mathbf{e}}^{2}\right)}
$$

Plugging in the values $\Xi_{T}=0.2-0.3 \mathrm{~cm}^{-2} \mathrm{~g}$ and $F_{\text {obs }}=0.2$ gives a constraint on the atomic parameter space [53]

$$
\left(\frac{0.1}{\alpha_{D}}\right)^{2}\left(\frac{1 \mathrm{GeV}}{\mu_{\mathbf{H}}}\right)^{2}\left(\frac{100 \mathrm{GeV}}{m_{\mathbf{H}}}\right) \lesssim(2-20) \frac{0.2-X_{\mathbf{e}}}{1-X_{\mathbf{e}}^{2}}
$$

Thus, we find that $X_{\mathbf{e}}$ and $\sigma / m_{\mathbf{H}}$ are bounded simultaneously. From our conservative (and representative) assumption about the Ion-Ion cross section, $X_{\mathbf{e}}$ is bounded to be less 


\section{CHAPTER 2. ATOMIC DARK MATTER}

than $10 \%-20 \%$ regardless of $\sigma / m_{\mathbf{H}}$. For very small $X_{\mathbf{e}}$, the usual CDM WIMP bounds on $\sigma / m_{\mathbf{H}}$ are applicable; our estimate yields $\sigma / m_{\mathbf{H}} \lesssim 1 \mathrm{~cm}^{2} / \mathrm{g}$, which is slightly larger than the detailed simulations of [54]. Figure 3.2 shows some of the allowed parameter space in the $\alpha_{D}-m_{\mathbf{e}}$ plane for a few atomic masses ranging between $10 \mathrm{GeV}$ and $1 \mathrm{TeV}$.

Previous considerations of a hidden $U(1)[33,34]$ have concluded that soft scattering of charged dark matter can drastically affect halo formation and thereby rule out large swaths of parameter space. This result follows from the soft singularity in the Rutherford scattering rate which, when integrated over galactic time scales, can lead to significant energy transfer between charged particles. This effect tends to smooth out the core of the dark matter distribution. Application of these results excludes all of the parameter space shown in Figure 3.2. However, since Hydrogen-Hydrogen scattering is well modelled by hardsphere scattering in the majority of the considered parameter space, these bounds are not applicable to atomic dark matter. The relevant bounds from halo formation considerations are $0.1 \mathrm{~cm}^{2} / \mathrm{g} \lesssim \sigma / m_{\mathbf{e}} \lesssim 1 \mathrm{~cm}^{2} / \mathrm{g}$, which do not signficantly change our conclusions [55]. Atomic dark matter provides a dynamical mechanism to shut off the naïve long range effects of a hidden $U(1)$.

\subsection{Direct Detection}

Atomic dark matter, as thus far considered, is secluded from the standard model. While the cosmology of atomic dark matter is interesting in its own right, it naturally lends itself 


\section{CHAPTER 2. ATOMIC DARK MATTER}

to inelastic scattering because of energy level quantization. This offers an exciting possible explanation of the DAMA data [9].

The unperturbed energy levels of hydrogen are

$$
E_{n}=\frac{\alpha_{D}^{2} \mu_{\mathbf{H}}}{2 n^{2}}
$$

One might hope that the DAMA scale $-\mathcal{O}(100 \mathrm{keV})$ - could be generated by energy differences between levels with different principle quantum numbers. Generically the rate of elastic scattering will be greater than that of inelastic scattering. However, predominantly inelastic scattering could be enforced by setting $m_{\mathbf{p}}=m_{\mathbf{e}}=2 \mu_{\mathbf{H}}$. In this case, the first Born term for elastic scattering vanishes. Unfortunately, efficient recombination in such a scenario forces one to consider $m_{\mathbf{H}} \sim \mathrm{GeV}$, which is too small to account for the recoil energies measured by DAMA. Nevertheless, atoms have a rich structure and the allowed parameter space for viable recombination naturally leads to hyperfine splittings on the order of $100 \mathrm{keV}$ for weak-scale hydrogen. The hyperfine splitting is given by

$$
E_{h f}=\frac{2}{3} g_{\mathbf{e}} g_{\mathbf{p}} \alpha_{D}^{4} \mu_{H} \frac{m_{\mathbf{e}}}{m_{\mathbf{p}}}
$$

where $g_{\mathbf{e}}, g_{\mathbf{p}}$ are the gyromagnetic ratios of the dark electron and dark proton, which we take to be equal to two.

Exploiting this scale requires a scattering process which is dependent on the spins of the dark atom's constituents. This can be accomplished with a broken $U(1)_{X}$ which is axially coupled to the dark matter and mixed with the standard model hypercharge as in [37]

$$
\mathcal{L}_{m i x}=\epsilon X^{\mu \nu} B_{\mu \nu}
$$




\section{CHAPTER 2. ATOMIC DARK MATTER}

Having an axial coupling in the dark sector and a vector coupling to the standard model will ensure that the dominant scattering process changes the dark atom spin state by one unit. After integrating out the $\mathrm{Z}$ boson and diagonalizing the gauge kinetic terms, the Lagrangian becomes

$$
\begin{aligned}
\mathcal{L} & =\mathcal{L}_{S M}+\mathcal{L}_{D M}+\mathcal{L}_{\text {Dark Gauge }} \\
\mathcal{L}_{D M} & =\bar{\Psi}_{\mathbf{p}}\left(i \not \partial-g_{5} \gamma_{5} X+g A+m_{\mathbf{p}}\right) \Psi_{\mathbf{p}}+\bar{\Psi}_{\mathbf{e}}\left(i \not \partial+g_{5} \gamma_{5} \not X-g A+m_{\mathbf{e}}\right) \Psi_{\mathbf{e}} \\
& -\frac{\epsilon s_{w}}{m_{Z}^{2}} J_{Z \mu} J_{D}^{\mu} \\
\mathcal{L}_{\text {Dark Gauge }} & =-\frac{1}{4} A_{\mu \nu}^{2}-\frac{1}{4} X_{\mu \nu}^{2}-\left(\epsilon c_{w} J_{E M}^{\mu}+\epsilon s_{w}\left(\frac{M_{X}}{m_{Z}}\right)^{2} J_{Z}^{\mu}\right) X_{\mu}+\frac{M_{X}^{2}}{2} X^{2} .
\end{aligned}
$$

The parameters $c_{w}$ and $s_{w}$ are the cosine and sine of the weak mixing angle. The dark current $J_{D}^{\mu}$ is

$$
J_{D}^{\mu}=-g_{5} \bar{\Psi}_{\mathbf{p}} \gamma^{\mu} \gamma_{5} \Psi_{\mathbf{p}}+g_{5} \bar{\Psi}_{\mathbf{e}} \gamma^{\mu} \gamma_{5} \Psi_{\mathbf{e}}
$$

and $J_{E M}^{\mu}$ and $J_{Z}^{\mu}$ are the standard model electromagnetic and weak neutral currents, respectively.

The calculation of the direct detection scattering cross section is organized as follows. First, we derive the non-relativistic interaction Hamiltonian for dark atoms and standard model nucleons from Eq. (2.3.26). Second, we use this to calculate the differential cross section for a dark atom to scatter from a spin singlet to a spin triplet state off of a standard model nucleon and append a form factor to account for recoil of the entire nucleus. Third, 


\section{CHAPTER 2. ATOMIC DARK MATTER}

we rewrite the resulting rate in terms of the nuclear recoil $E_{R}$. Finally, we convolve the recoil rate with the dark matter velocity distribution.

\subsubsection{Non-relativistic Interaction Hamiltonian}

In order to calculate the scattering cross section for dark atoms off of standard model nuclei we derive the interaction Hamiltonian by taking the non-relativistic limit of the currentcurrent interaction

$$
\mathcal{A}=\left\langle J_{D}^{\mu} D_{\mu \nu} J_{Y}^{\nu}\right\rangle
$$

where $D_{\mu \nu}$ is the Coulomb gauge propagator for $X$. The leading behavior of Eq. (2.3.28) is

$$
\begin{aligned}
\mathcal{A} & \simeq \frac{g_{5} \epsilon c_{w} e}{\vec{q}^{2}+M_{X}^{2}} \chi_{s^{\prime}}^{\prime \dagger}\left[\frac{\vec{\sigma}_{\mathbf{e}} \cdot \vec{p}_{\mathbf{e}}}{m_{\mathbf{e}}}+\frac{\vec{\sigma}_{\mathbf{e}} \cdot \vec{q}}{2 m_{\mathbf{e}}}+\frac{\vec{\sigma}_{\mathbf{e}} \cdot \vec{q}}{2 m_{n}}-\frac{\vec{\sigma}_{\mathbf{e}} \cdot \vec{p}_{n}}{m_{n}}+\frac{\vec{\sigma}_{\mathbf{p}} \cdot \vec{p}_{n}}{m_{n}}-\frac{\vec{\sigma}_{\mathbf{p}} \cdot \vec{q}}{2 m_{n}}\right. \\
& \left.+\frac{\left(\vec{\sigma}_{\mathbf{e}} \cdot \vec{q}\right)\left(\vec{q} \cdot \vec{p}_{n}\right)}{m_{n}\left(\vec{q}^{2}+M_{X}^{2}\right)}-\frac{\left(\vec{\sigma}_{\mathbf{e}} \cdot \vec{q}\right) \vec{q}^{2}}{2 m_{n}\left(\vec{q}^{2}+M_{X}^{2}\right)}-\frac{\left(\vec{\sigma}_{\mathbf{p}} \cdot \vec{q}\right)\left(\vec{q} \cdot \vec{p}_{n}\right)}{m_{n}\left(\vec{q}^{2}+M_{X}^{2}\right)}+\frac{\left(\vec{\sigma}_{\mathbf{p}} \cdot \vec{q}\right) \vec{q}^{2}}{2 m_{n}\left(\vec{q}^{2}+M_{X}^{2}\right)}\right] \chi_{s} \xi_{r^{\prime}}^{\dagger} \xi_{r} .
\end{aligned}
$$

where $m_{n}$ is the nucleon mass, $\vec{p}_{\mathbf{e}}$ is the initial momentum of the dark electron, that of the nucleon is $\vec{p}_{n}$ and $\vec{q}$ is the momentum conjugate to the relative coordinate between the electron and nucleon. $\chi_{s}$ and $\chi_{s^{\prime}}$ are the initial and final spin states of the atom and can be written in the form: $\chi_{\text {Atom }}=\chi_{\mathbf{p}} \otimes \chi_{\mathbf{e}}$. The dark matter spin operators are $\vec{S}_{e, p}=$ $\mathbb{1} \otimes \vec{\sigma}_{\mathbf{e}} / 2, \vec{\sigma}_{\mathbf{p}} / 2 \otimes \mathbb{1} . \xi_{r}$ and $\xi_{r^{\prime}}$ are the initial and final spin states of the standard model nucleon. In the following analysis we consider proton masses of $\mathcal{O}(100 \mathrm{GeV})$ and electron 


\section{CHAPTER 2. ATOMIC DARK MATTER}

masses of $\mathcal{O}(1 \mathrm{GeV})$, so we have ignored terms suppressed by $m_{\mathbf{p}}$. We have also dropped terms suppressed by $M_{Z}$. Finally, we have omitted terms which depend on the spin of the standard model nucleon, as we expect these terms to contribute incoherently to the overall scattering cross section and hence be suppressed by the atomic number of the nucleus.

\subsubsection{Inelastic Dark Atom - Nucleus Scattering}

The cross section in the center of mass of the hydrogen-nucleus system is given by $[58,59]$

$$
\frac{d \sigma_{h f}}{d \Omega} \equiv \frac{d \sigma}{d \Omega}(S=0 \rightarrow S=1)=\frac{\mu_{N A}^{2}}{4 \pi^{2}}\left|\frac{k^{\prime}}{k}\right|\left|\left\langle\mathbf{p}_{\mathbf{H}}^{\prime}, \mathbf{p}_{N}^{\prime}, N^{\prime}, H^{\prime}\left|\hat{H}_{\text {int }}\right| \mathbf{p}_{\mathbf{H}}^{\prime}, \mathbf{p}_{N}, N, H\right\rangle\right|^{2}
$$

Here $\hat{H}_{\text {int }}$ is the interaction Hamiltonian, which is obtained from Eq. 2.3.29) by Fourier transforming $\vec{q}$ to position space. The prefactor contains $\mu_{N A}=\left(m_{\mathbf{H}}+M_{N}\right) /\left(m_{\mathbf{H}}+\right.$ $\left.M_{N}\right)$ which is the reduced mass of hydrogen and the nucleus, and the momenta $\vec{k}$ and $\vec{k}^{\prime}$ which are the initial and final momenta conjugate to the relative coordinate between the atom and the nucleus. Our basis is the hydrogen atom momentum $\left\{\mathbf{p}_{\mathbf{H}}, \mathbf{p}_{\mathbf{H}}^{\prime}\right\}$, the nucleus

momentum, $\left\{\mathbf{p}_{N}, \mathbf{p}_{N}^{\prime}\right\}$, and the internal states of hydrogen and the nucleus, $\left\{A, A^{\prime}\right\}$ and $\left\{N, N^{\prime}\right\}$. The explicit evulation of the matrix element in Eq. (2.3.30) is complicated by the fact that there are four particles in the incoming and outgoing states. The hydrogen atom and the nucleus are both free particles while the electron and nucleon are bound to the respective free particle motion. Since the scattering centers, the electron and nucleon, 


\section{CHAPTER 2. ATOMIC DARK MATTER}

do not correspond to the coordinates of free particle motion, hydrogen's center of mass and the nucleus's center of mass, the matrix element in Eq. (2.3.30) contains an atomic formfactor in addition to the usual nuclear form factor. We find, ignoring terms suppressed by $m_{\mathbf{e}} / m_{\mathbf{H}}$ and $m_{n} / M_{N}$, the cross section to be

$$
\begin{aligned}
\frac{d \sigma_{h f}}{d \Omega} & =\left|\frac{k^{\prime}}{k}\right|\left(\frac{2 \mu_{N A}^{2}}{\pi^{2}}\right)\left(\frac{g_{5} Z e \epsilon c_{w}}{q^{2}+M_{X}^{2}}\right)^{2} \mid \chi_{s^{\prime}}^{\prime \dagger}\left[F_{e l}\left(q^{2}\right) \frac{\vec{S}_{\mathbf{e}} \cdot \vec{q}}{\mu_{n e}}-F_{e l}\left(q^{2}\right) \frac{\left(2 \vec{S}_{\mathbf{e}} \cdot \vec{q}\right) q^{2}}{m_{n}\left(q^{2}+M_{X}^{2}\right)}\right. \\
& \left.-\frac{\vec{S}_{\mathbf{p}} \cdot \vec{q}}{m_{n}}+\frac{\left(2 \vec{S}_{\mathbf{p}} \cdot \vec{q}\right) q^{2}}{m_{n}\left(q^{2}+M_{X}^{2}\right)}\right]\left.\chi_{s}\right|^{2}\left|F_{\mathbf{H}}\left(q^{2}\right)\right|^{2}
\end{aligned}
$$

The electron form factor $F_{e l}\left(q^{2}\right)$ is found to be

$$
F_{e l}\left(q^{2}\right)=\left\langle 0\left|e^{i \vec{q} \cdot \vec{r}}\right| 0\right\rangle=\left(1+\frac{q^{2} a_{0}^{2}}{4}\right)^{-2}
$$

where $|0\rangle$ is the ground state of the dark atom. The function $F_{H}\left(q^{2}\right)$ is the Helm nuclear form factor which accounts for the overlap between nucleon and nuclear states $[60,61]$. We have averaged over initial nucleon spin and summed over final nucleon spin. Summing over the final atomic spin states and using Eq. (2.3.32) we have

$$
\frac{d \sigma_{h f}}{d \Omega}=\frac{M_{N}^{2}}{2}\left|\frac{k^{\prime}}{k}\right|\left(\frac{g_{5} Z e \epsilon c_{w}}{\pi}\right)^{2}\left(\frac{F_{e l} F_{H} G\left(q^{2}\right)}{M_{X}^{2}\left(1+\frac{q^{2}}{M_{X}^{2}}\right)}\right)^{2}\left(\frac{q}{\mu_{n e}}\right)^{2}
$$

we have defined the function $G\left(q^{2}\right)$ as follows

$$
G\left(q^{2}\right) \equiv 1+\frac{\mu_{n e}}{m_{n}}\left[F_{e l}^{-1}-\left(1+F_{e l}^{-1}\right) \frac{q^{2}}{q^{2}+M_{X}^{2}}\right] .
$$

We rewrite the above in the following form

$$
\begin{aligned}
\frac{d \sigma_{h f}}{d E_{R}} & =\frac{4 Z^{2} \alpha}{\mu_{n e}^{2} f_{\text {eff }}^{4}} \frac{M_{N}^{2}}{v_{r e l}^{2}} \frac{E_{R} F_{H}^{2} F_{e l}^{2}}{\left(1+2 M_{N} E_{R} / M_{X}^{2}\right)^{2}} G^{2}\left(E_{R}\right) \\
f_{e f f}^{4} & \equiv \frac{M_{X}^{4}}{2\left(g_{5} \epsilon c_{w}\right)^{2}}
\end{aligned}
$$

where we have defined the scale $f_{\text {eff }}$ for compactness and for comparison to Ref. [37]. 


\section{CHAPTER 2. ATOMIC DARK MATTER}

\subsubsection{Modulated Nuclear Recoil Rate}

The amplitude of the modulated recoil rate at a detector with $N_{T}$ target nuclei of mass $M_{N}$ is given by

$$
\left.\frac{d R}{d E_{R}} \equiv \frac{1}{2} N_{T} \frac{\rho}{m_{D M}} \int_{v_{\min }}^{v_{\text {esc }}} d v v f(v) \frac{d \sigma_{h f}}{d E_{R}}\right|_{\text {January }} ^{\text {June }},
$$

where $v$ is the relative velocity between the dark atom and the nucleus, $\rho=0.3 \mathrm{GeV} / \mathrm{cm}^{3}$ is the local dark matter density and we use $v_{e s c}=550 \mathrm{~km} / \mathrm{s}$ for the dark matter escape velocity - see Ref. [62]. The lower bound on the velocity integration is the minimum relative velocity that can produce a given recoil energy

$$
v_{\text {min }} \equiv \sqrt{\frac{1}{2 M_{N} E_{R}}}\left(\frac{M_{N}+M_{\mathbf{H}}}{M_{\mathbf{H}}} E_{R}+E_{h f}\right) .
$$

Following Ref's. [62,63], the velocity distribution in Eq. (2.3.37) is approximated by

$$
f(v)= \begin{cases}\frac{1}{N}\left(\frac{1}{\pi v_{0}^{2}}\right)^{3 / 2} e^{-v^{2} / v_{0}^{2}}, & \text { for } v<v_{e s c} \\ 0, & \text { for } v \geq v_{e s c}\end{cases}
$$

The normalization is

$$
N=\operatorname{erf}\left(\frac{v_{e s c}}{v_{0}}\right)-\frac{2}{\sqrt{\pi}} \frac{v_{e s c}}{v_{0}} e^{-\left(v_{e s c} / v_{0}\right)^{2}}
$$

with $v_{0}=220 \mathrm{~km} / \mathrm{s}$.

Figure 2.2 is an example of the modulated count rate at DAMA as defined in Eq. 2.3.37) with the data points and reported uncertainties from DAMA and DAMA/LIBRA [9]. We have plotted the modulated spectrum for three choices of the set 


\section{CHAPTER 2. ATOMIC DARK MATTER}

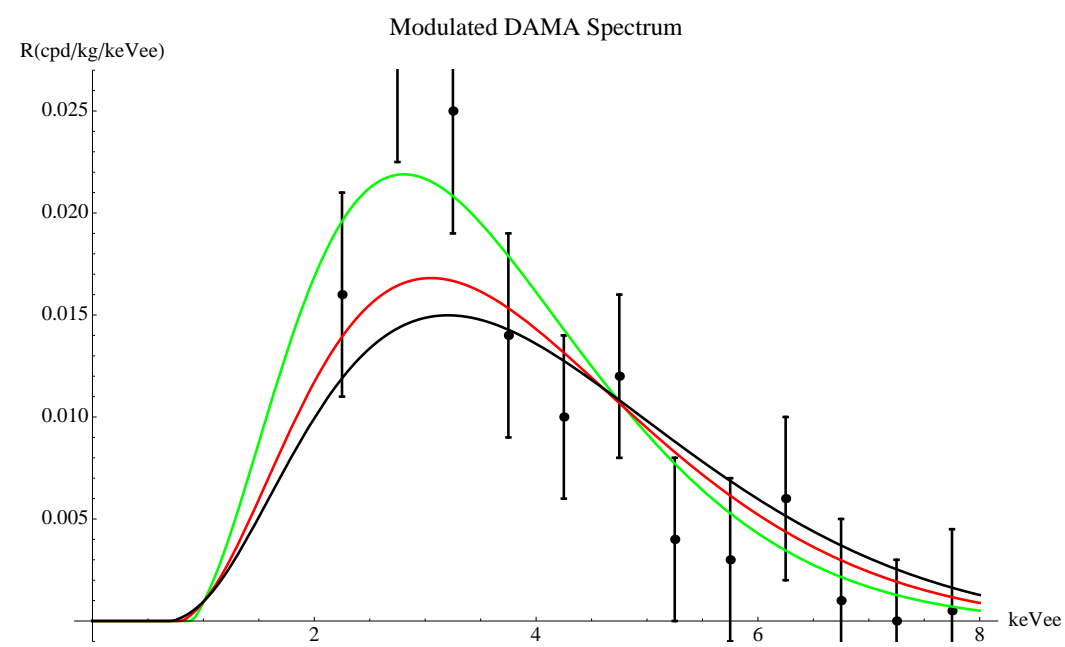

Figure 2.2: Examples of the modulated spectrum at DAMA defined in Eq. 2.3.37) complete with the data points from the DAMA and DAMA/LIBRA experiments. The curves correspond to the following choice of parameters from left to right in order of their rise from zero (black, red and green in color order) $m_{\mathbf{p}}=200,100,70 \mathrm{GeV} ; m_{\mathbf{e}} \simeq$ $1.59,1.53,2.15 \mathrm{GeV} ; f_{\text {eff }} \simeq 89,109,111 \mathrm{GeV}$. The hyperfine splittings are all about $97 \mathrm{keV}$.

of parameters $\left(m_{\mathbf{p}}, m_{\mathbf{e}}, \alpha_{D}, M_{X}, g_{5}\right.$ and $\left.\epsilon\right)$ which satisfy the rather stringent list of constraints enumerated below. Note the linear dependence on $E_{R}$ and the presence of an atomic form factor in Eq. 2.3.35). Although the first term tends to push the peak toward larger values of $E_{R}$, the atomic form factor turns off scattering when $q a_{0} \sim 1$.

\section{Mixing With The Standard Model:}

Perhaps the harshest constraints are on the mass of the axial $U(1)$ and its kinetic mixing with the standard model, since the direct detection cross section is roughly proportional to $\epsilon^{2} / M_{X}^{4}$. The one loop contribution of $X$ to the anomolous magnetic moment of the muon 


\section{CHAPTER 2. ATOMIC DARK MATTER}

is

$$
a_{\mu}^{X}=\frac{\alpha \epsilon^{2}}{2 \pi} \int_{0}^{1} d z \frac{2 m_{\mu}^{2} z(1-z)^{2}}{m_{\mu}^{2}(1-z)^{2}+m_{X}^{2} z} .
$$

As discussed in Ref. [109], regardless of how one treats the hadronic contribution to the theoretical prediction of $a_{\mu}$, the $X$ boson's one loop contribution must satisfy

$$
a_{\mu}^{X} \leq 7.4 \times 10^{-9}
$$

In order to be conservative we restrict ourselves to (see Figure 1 in Ref. [109])

$$
M_{X} \geq 100 \mathrm{MeV} \text { and } \epsilon^{2} \lesssim 10^{-5}
$$

Mixing between the massless gauge boson in the dark sector and the photon is not induced by loops in our theory, and we nominally set it zero. A constraint on this mixing, $\epsilon^{\prime}$, can be derived from bounds on its contribution to the anomalous magnetic moment of the electron. The constraint is $\epsilon^{\prime}<\mathcal{O}\left(10^{-4}\right)$ [109]. Astrophysical constraints also exist, but are much less restrictive for the range of electron masses we are considering [65].

\section{Sufficient Recombination:}

The residual ionized dark matter will scatter elastically as it does not cost any energy to flip a free spin. Efficient recombination and a hyperfine splitting consistent with DAMA imply that the typical electron mass is $\mathcal{O}(1 \mathrm{GeV})$ and therefore too small to induce observable nuclear recoils. The strongest constraints on direct detection of the free dark protons come from CDMS $[24,66]$. With a net exposure of $174.7 \mathrm{~kg}-\mathrm{d}$, the CDMS experiment 


\section{CHAPTER 2. ATOMIC DARK MATTER}

allows 5.3 signal events at $90 \%$ confidence level. To be consistent with the bounds from direct detection $\prod_{4}$ we demand that $X_{\mathbf{e}} \leq 10^{-4}$.

\section{Energy Level Corrections Due to the Axial U(1):}

The proton-electron interaction due to the broken axial $\mathrm{U}(1)$ is a perturbation to the hydrogen Hamiltonian and gives a correction to the hyperfine level splitting. The correction is given by

$$
\delta E_{h f} \sim\left\langle 0\left|\frac{g_{5}^{2}}{4 \pi} \frac{e^{-M_{X} r}}{r}\right| 0\right\rangle=\frac{g_{5}^{2}}{4 \pi a_{0}}\left(1+a_{0} M_{X}\right)^{-2} .
$$

Requiring $\delta E_{h f} \ll E_{h f}$ gives

$$
g_{5}^{2} \ll \frac{32 \pi}{3} \alpha_{D}^{3} \frac{m_{e}}{m_{\mathbf{p}}}\left(1+\frac{M_{X}}{\alpha_{D} \mu_{H}}\right)^{2} .
$$

The parameter sets shown in Figure 2.2 satisfy this constraint.

\section{Breaking the Axial U(1):}

Masses for the dark electron, dark proton and the axial gauge boson all violate the axial U(1) symmetry. Perhaps the simplest way to give mass to these particles is giving a vev to a charge +2 scalar $\phi_{+2}$, as in [37]

$$
\begin{aligned}
\mathcal{L} & \ni\left|D_{5}^{\mu} \phi_{+2}\right|^{2}-\lambda\left(\left|\phi_{+2}\right|^{2}-v_{+2}^{2}\right)^{2}+y_{p}\left(\bar{p}_{R} \phi_{+2} p_{L}+\bar{p}_{L} \phi_{+2}^{*} p_{R}\right) \\
& +y_{e}\left(\bar{e}_{R} \phi_{+2} e_{L}+\bar{e}_{L} \phi_{+2}^{*} e_{R}\right) .
\end{aligned}
$$

\footnotetext{
${ }^{4}$ The discussion in this section actually puts a bound on the local ionized fraction. We assume for simplicity - here and throughout this chapter - that the distribution of ionized dark matter matches that of atomic dark matter. However, due to the presence of a long-ranged force, it may be that the ionized distribution is very different from the typical dark matter halo. A full N-body simulation of a multiple species halo is beyond the scope of the present work, so for now we ignore this interesting possibility.
} 


\section{CHAPTER 2. ATOMIC DARK MATTER}
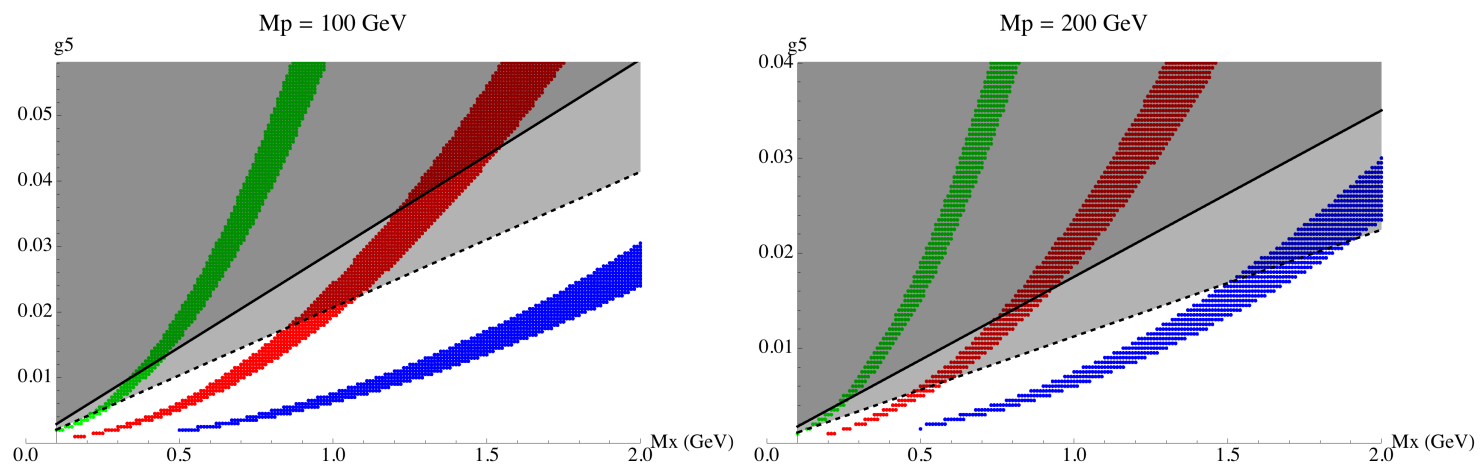

Figure 2.3: The allowed parameter space for $M_{X}$ and $g_{5}$ for two values of the dark proton mass. The other atomic parameters $-\alpha_{D}$ and $m_{\mathbf{e}}$ - have been chosen so that $E_{h f} \simeq 97 \mathrm{keV}$ and $X_{\mathbf{e}} \leq 10^{-4}$. The allowed parameter space for $M_{X}$ and $g_{5}$ for two values of the dark proton mass. The other atomic parameters $-\alpha_{D}$ and $m_{\mathbf{e}}$ - have been chosen so that $E_{h f} \simeq$ $97 \mathrm{keV}$ and $X_{\mathbf{e}} \leq 10^{-4}$. On each plot, the displayed values of $\epsilon^{2}$ are $10^{-6}, 10^{-5}$ and $10^{-4}$ from left to right (green, red and blue in color order). To be consistent with constraints discussed in Ref. [109], $M_{X} \geq 0.5 \mathrm{GeV}$ for $\epsilon^{2}=10^{-4}$. The allowed points have an average rate, in the 2 to $6 \mathrm{keVee}$ bins at DAMA, between $0.99 \times 10^{-2}$ and $1.63 \times 10^{-2} \mathrm{cpd} / \mathrm{kg} / \mathrm{keVee}$. The excluded regions correspond to choices of parameters which do not satisfy Eq. (2.3.46); the solid black line is for $\Lambda=1 \mathrm{TeV}$ and the dashed line is for $\Lambda=10 \mathrm{TeV}$.

When the scalar is at its vev, the mass spectrum is

$$
\begin{aligned}
M_{X} & =g_{5} v_{+2} \\
m_{\mathbf{p}} & =y_{\mathbf{p}} v_{+2} \\
m_{\mathbf{e}} & =y_{\mathbf{e}} v_{+2} .
\end{aligned}
$$

The DAMA signal requires $m_{\mathbf{p}}>m_{X}$ and $g_{5} \gtrsim \mathcal{O}\left(10^{-2}\right)$ thus one might worry about the perturbativity of $y_{\mathbf{p}}$. The yukawa coupling runs according to the following one loop renormalization group evolution [67]

$$
y_{\mathbf{p}}(\Lambda)=\sqrt{\frac{2 \pi^{2}}{\ln \left(\Lambda / m_{\mathbf{p}}\right)}},
$$

which blows up at the scale $\Lambda$. If we take $\Lambda=1 \mathrm{TeV}$ or $10 \mathrm{TeV}$, our parameter space is 


\section{CHAPTER 2. ATOMIC DARK MATTER}

constrained as shown in Figure 2.3. In principle, the proton could be a composite object and the axial-symmetry breaking could occur at strong coupling (as in QCD) and not via a weakly coupled scalar. The proton could also carry a charge under another gauge interaction that is relatively strong, but breaks at a TeV, thus tempering the $\mathrm{UV}$ behavior of $y_{\mathbf{p}}$. We leave explicit models of UV completions to future work.

Figure 2.3 displays the allowed parameter space for a few choices of $M_{X}, g_{5}$ and $\epsilon$ with $X_{\mathbf{e}} \leq 10^{-4}$ level.

\subsection{Discussion}

Dark matter succinctly explains a number of astrophysical and cosmological observations that are otherwise puzzling. Standard WIMP dark matter can accommodate the gross features of these observations and naturally exists in models that attempt to explain the origin of the weak-scale. However, the typical WIMP seems unable to explain tensions between direct detection experiments. These considerations point to the possibility of a non-minimal dark sector, which contains more similarities to the light sector than is typically thought. Atomic dark matter - with a non-negligible ionized fraction $X_{\mathbf{e}}$ and a new massless gauge boson - offers the possibility of significantly different phenomena in the dark sector than those of standard WIMPs.

Atomic dark matter may have hyperfine transitions of the right size to offer an inelastic explanation for the DAMA data. If our simple model of atomic dark matter is the right 


\section{CHAPTER 2. ATOMIC DARK MATTER}

explanation for DAMA and the ionized components of the halo follow the distribution of the atomic dark matter, then other direct detection experiments should see dark protons in the near future. Simulations of stucture formation with charged and neutral components could shed light on these issues.

The dynamics that lead to atomic dark matter also may have other phenomenological implications. For example, in parts of parameter space where the ionized fraction is large enough, $\mathbf{H}_{2}$ molecules may form through processes catalyzed by the residual ions, as in the SM [70]

$$
\begin{aligned}
\mathbf{H}+\mathbf{e} & \leftrightarrow \mathbf{H}^{-}+\gamma \\
\mathbf{H}^{-}+\mathbf{H} & \leftrightarrow \mathbf{H}_{2}+\mathbf{e}
\end{aligned}
$$

and

$$
\begin{aligned}
\mathbf{H}+\mathbf{p} & \leftrightarrow \mathbf{H}_{2}^{+}+\gamma \\
\mathbf{H}_{2}^{+}+\mathbf{H} & \leftrightarrow \mathbf{H}_{2}+\mathbf{p} .
\end{aligned}
$$

The existence of molecular states in the dark sector offers the possibility of cooling mechanisms which, in the SM, are thought to be very important for the formation of the first stars [71]. This raises the interesting question of whether and to what extent compact objects, e.g. dark stars, could form for weak-scale dark atoms. Moreover, if the dark photon mixes with the SM photon, it may result in dark atomic line emissions in cosmic gamma rays.

We have presented a somewhat generic model of atomic dark matter. Explicit models 


\section{CHAPTER 2. ATOMIC DARK MATTER}

which explain the asymmetry abundance and which serve as ultraviolet completions of the model could potentially relate astrophysical phenomena to physics to be probed by the Large Hadron Collider. The part of parameter space in which the measured DAMA signal is post-dicted requires the dark proton to be strongly coupled, or nearly so, at a $\mathrm{TeV}$. If strongly coupled, one could imagine additional features of the dark sector - i.e., a composite atomic nucleus - which more strongly mimic our visible world. 


\section{Chapter 3}

\section{UV Completion: Dark Atoms}

\subsection{Introduction}

While indirect cosmological observations provide abundant evidence for the existence of dark matter (DM) [?, 4, 21], terrestrial evidence of its particle nature has been elusive. The identity of DM stands alongside several important open questions at the intersection of cosmology and particle physics including the missing anti-matter, the number of light degrees of freedom in the CMB [72], and the observed absence of small-scale structure $[73,74]$.

Recently, the CoGeNT direct detection experiment [75] reported an excess of events in their low-recoil bins. If this excess is interpreted as evidence of a DM particle, the natural scale for its mass is $\sim \mathcal{O}(10 \mathrm{GeV})$. Since this energy scale does not easily fit the so-called WIMP paradigm, the dark sector must generically be expanded to generate the right cosmological 


\section{CHAPTER 3. UV COMPLETION: DARK ATOMS}

abundance. This can be accomplished with new light states as in [76-78] or by relating the DM abundance to the SM baryon asymmetry as in [79-83, 85-93, 133]. See [94] for a thorough treatment of the constraints on such models.

Generic models of light DM are highly constrained by the null results of CDMS [95] and XENON10 $[25,96,97]$ experiments. The CDMS collaboration has recently reanalyzed the CDMS II Germanium data with the detection threshold lowered to $2 \mathrm{keV}$. This analysis excludes both the DAMA [98] and CoGeNT preferred regions for WIMP DM. XENON10 also claims to rule out the WIMP interpretation of DAMA and CoGeNT, though there is controversy over XENON's scintillation efficiency $\mathcal{L}_{\text {eff }}$ at low energies [99-101]. A theory which can explain the positive signals while evading all the constraints may require some or all of the following epicycles: additional dark species [102], momentum dependent DM/SM interactions [103] or non-standard couplings to nucleons [104] (see Ref. [105] for a thorough study).

In this note, we suggest that atomic dark matter $(\mathrm{aDM})$ may answer a number of important, open questions in cosmology. We find that aDM can generate the right DM abundance and baryon asymmetry, contains additional relativistic degrees of freedom and is capable of smoothing structure on much larger scales than conventional CDM candidates [106]. Furthermore, aDM may reconcile CoGeNT with constraints from null experiments. We also find that the regions of aDM parameter space favored by CoGeNT are consistent with preliminary signals at CRESST [107]. Finally, we note that that the existence of both dark 


\section{CHAPTER 3. UV COMPLETION: DARK ATOMS}

ions and atoms within aDM gives rise to a unique halo structure.

Section 3.2 gives a brief overview of aDM; section 3.3.2 extends the simple framework to explain both the dark matter abundance and the SM baryon asymmetry via the mechanism recently proposed in [92]; section 3.3.3 describes and justifies the pattern of spontaneous symmetry breaking in the dark sector; section 3.3 .4 describes the recombination of multiple species of dark atoms; section 3.4 reviews relevant direct detection signals, limits and constraints on the aDM parameter space; subsection 3.4.1 includes a discussion of the novel aDM halo structure; finally, section 3.5 summarizes our results and outlines future directions.

\subsection{Review of aDM}

Atomic dark matter consists of four Weyl fermions - $\mathbf{E}, \mathbf{E}^{c}, \mathbf{P}$ and $\mathbf{P}^{c}$ - charged under two $U(1)$ 's. The first, $U(1)_{D}$, has vector couplings and is unbroken. The second, $U(1)_{X}$, has axial-vector couplings and is spontaneously broken by the vev of $\mathcal{X}$ which is also responsible for the masses of $\mathbf{E}$ and $\mathbf{P}$.

The axial gauge boson is kinetically mixed with $\mathrm{SM} U(1)_{Y}$ through a coupling of the form [108]

$$
\mathcal{L}_{\text {mix }}=\frac{\epsilon}{2} B_{\mu \nu} X^{\mu \nu}
$$

This operator arises from integrating out a heavy fermion with vector couplings to both 


\section{CHAPTER 3. UV COMPLETION: DARK ATOMS}

\begin{tabular}{|c|c|c|}
\hline & $U(1)_{D}$ & $U(1)_{X}$ \\
\hline $\mathbf{E}$ & -1 & -1 \\
$\mathbf{E}^{c}$ & 1 & -1 \\
$\mathbf{P}$ & 1 & 1 \\
$\mathbf{P}^{c}$ & -1 & 1 \\
$\mathcal{X}$ & 0 & 2 \\
\hline
\end{tabular}

Table 3.1: Field content and $U(1)$ charges for aDM.

$U(1)$ 's so $\epsilon$ is given by:

$$
\epsilon(\mu)=\frac{g_{Y} g_{X}}{16 \pi^{2}} \ln \left(\frac{M_{\text {heavy }}}{\mu}\right)
$$

where experimental constraints allow $\epsilon^{2} \lesssim 10^{-5}$ for $M_{X} \gtrsim 400 \mathrm{MeV}$ [109-111]. Note that the existence of a $U(1)$ gauge boson with this mass and coupling can ameliorate the discrepancy between the standard model prediction and the measured value of the muon g-2 [109]. The field content and interactions above are capable of producing a successful cosmology and unique direct detection spectrum.

\subsubsection{Cosmology}

The possibility of $U(1)$ charged DM with long-range interactions, has been explored in a number of works $[33-36,106]$. In the case where DM exists in ionic form, halo morphology and bullet-cluster observations $[53,54]$ place tight constraints on the $\left(\alpha_{D}, m_{D M}\right)$ 


\section{CHAPTER 3. UV COMPLETION: DARK ATOMS}

parameter space. Long-range interactions push the DM from a virial configuration toward kinetic equilibrium and can make the scattering rate in the bullet cluster too high. The aDM scenario avoids these problems by assembling the dark ions into atomic bound states which are net neutral under the $U(1)$ with a smaller fraction $X_{E}$ existing in ionic form. The ionic fraction is defined as:

$$
X_{E} \equiv \frac{n_{E}}{n_{E}+n_{H}}
$$

and it is most sensitive to the value of $\alpha_{D}$, tending to decrease as the coupling increases. Similarly, $X_{E}$ also tends to decrease as $m_{E}$ increases with $m_{P}$ held fixed. The dependence on $m_{P}$ is much weaker than the other two parameters. See Figure 3.2 for the light atoms considered in this work and Figure 1 in Ref. [106] for a more general treatment.

In this framework, the cosmological abundance of DM is dependent upon the existence of an asymmetry between $(E, P)$ and $(E, P)^{c}$ and we return to the question of generating this asymmetry in Section 3.3 .

\subsubsection{Direct Detection}

The leading interaction between $\mathrm{aDM}$ and the $\mathrm{SM}$ is through the $X-\gamma$ mixing in Eq. (3.2.1). The static potential between a SM particle with charge $Q_{\mathrm{EM}}$ and a DM ion with charge $Q_{X}$ goes like

$$
V\left(\vec{S}_{\mathrm{DM}}, \vec{r}\right) \sim\left(\epsilon Q_{X} Q_{\mathrm{EM}}\right)\left(\vec{S}_{\mathrm{DM}} \cdot \vec{r}\right) \frac{e^{-M_{X} r}}{r^{2}}
$$




\section{CHAPTER 3. UV COMPLETION: DARK ATOMS}

with the dependence on the DM spin-operator arising from the axial-vector couplings of $\mathrm{U}(1)_{X}$, cf. Ref. [37]. As in SM hydrogen, the aDM ground state is the $n=1$ state with anti-aligned spins and the $S=1$ triplet states have a slightly higher energy so there is a hyperfine splitting. At leading order, the interaction in Eq. 3.2.4 forces dark atoms to scatter inelastically from SM nuclei by excitation into the hyperfine state. The ratio of the hyperfine splitting $E_{\mathrm{hf}}$ to the ground state binding energy $B$ scales as

$$
\frac{E_{\mathrm{hf}}}{B} \propto \alpha_{D}^{2} \frac{m_{E}}{m_{P}}
$$

so that $E_{\mathrm{hf}}$ can easily be $\mathcal{O}(\mathrm{keV})$ for atomic masses $\mathcal{O}(10 \mathrm{GeV})$. This implies that dark ions, which are free spins, will scatter elastically such that the ionic recoil spectrum vanishes for small recoil energies. Thus, aDM realizes many of the mechanisms [105] necessary for reconciling CoGeNT with other null searches. In Section 3.4 we show that aDM can explain the positive signals reported by both CoGeNT and CRESST while evading bounds set by XENON and CDMS.

\subsection{Asymmetric Atomic Dark Matter}

In this section we propose an ultraviolet completion to the above model. It both dynamically explain the generation of the dark matter abundance (by linking it to the baryon asymmetry), and relieves the issue of a Landau pole for the $U(1)$ dark gauge field below the Planck scale. 


\section{CHAPTER 3. UV COMPLETION: DARK ATOMS}

\subsubsection{The Model}

We propose a nonabelian dark sector with $S U(2)_{D} \times U(1)_{X}$ gauge symmetry, where the labels $D$ and $A$ refer to "dark" and "axial," respectively. By embedding $U(1)_{D}$ into a non-Abelian group we avoid a Landau pole below the Planck scale. The matter Lagrangian contains

$\mathcal{L} \supset-\frac{1}{2} M_{n}^{i} n_{i}^{2}+y^{i j} n_{i} \ell_{j} h+\lambda_{\mathbf{e}}^{i} n_{i} E \varphi_{\mathbf{e}}+\lambda_{\mathbf{p}}^{i} n_{i} P \varphi_{\mathbf{p}}+y_{e} \mathcal{X} E E^{c}+y_{p} \mathcal{X}^{\dagger} P P^{c}+$ H.c., (3.3.6)

where $\ell_{j}, h$ are the Standard Model lepton and Higgs doublets; the $n_{i}$ (for $i=1,2$ ) are sterile neutrinos with GUT scale Majorana masses $M_{i}$; the $\varphi_{\mathbf{p}}, \varphi_{\mathbf{e}}$, and $\mathcal{X}$ are scalar fields. All gauge representations and quantum numbers are given in Table 3.2 .

For at least two species of sterile neutrinos, the parameters $y^{i j}$ and $\lambda_{e, p}^{i}$ contain irreducible complex-phases and give rise to $\mathrm{CP}$ violation. Out of equilibrium $n$ decays generate both the Standard Model lepton asymmetry and the asymmetric dark matter abundance. While lepton number is explicitly violated by neutrino Majorana masses, it remains a good accidental symmetry in the visible sector above the electroweak scale. In the dark sector, we impose a $\mathcal{Z}_{2}$ symmetry to dangerous $E P$ mass terms which allow EP annihilation into dark radiation, see Table 3.2. Notice that Eq. (3.3.6) does not allow explicit mass terms for the fermions $E$ and $P$; however, dark-sector symmetry breaking via the $\operatorname{VEV}\langle\mathcal{X}\rangle \equiv v_{\mathcal{X}}$ induces these fermion masses through the $\mathcal{X} E E^{c}$ and $\mathcal{X}^{\dagger} P P^{c}$ yukawa terms, as we will see in Section 3.3.3. 


\begin{tabular}{|c|c|c|c|}
\hline & $S U(2)_{D}$ & $U(1)_{X}$ & $\mathcal{Z}_{2}$ \\
\hline $\mathbf{E}$ & $\square$ & -1 & -1 \\
$\mathbf{E}^{c}$ & $\square$ & -2 & -1 \\
$\varphi_{\mathbf{e}}$ & $\square$ & 1 & -1 \\
$\mathbf{P}$ & $\square$ & 1 & 1 \\
$\mathbf{P}^{c}$ & $\square$ & 2 & 1 \\
$\varphi_{\mathbf{p}}$ & $\square$ & -1 & 1 \\
$\mathcal{X}$ & $\square$ & 3 & 1 \\
\hline
\end{tabular}

Table 3.2: Field content and gauge representations for Asymmetric aDM. The $U(1)_{X}$ charge assignments forbid $n E^{c} \varphi_{\mathbf{e}}$ and $n P^{c} \varphi_{\mathbf{p}}$ terms which would wash out the dark matter asymmetry. The discrete $\mathcal{Z}_{2}$ parity prevents atomic annihilation in the low energy effective theory. Mixing between $U(1)_{Y}$ and $U(1)_{D}$ is naturally tiny due to the $S U(2)_{D}$ embedding.

\subsubsection{Connecting Atomogenesis to Leptogenesis}

Following [92] and [93], we track the evolution of these asymmetries with the parameters

$$
\begin{aligned}
\epsilon_{\ell} & =\frac{\Gamma\left(n_{1} \rightarrow l h\right)-\Gamma\left(n_{1} \rightarrow \bar{l} h^{\dagger}\right)}{\Gamma_{n_{1}}} \\
\epsilon_{E} & =\frac{\Gamma\left(n_{1} \rightarrow E \varphi_{\mathbf{e}}\right)-\Gamma\left(n_{1} \rightarrow \bar{E} \varphi_{e}^{\dagger}\right)}{\Gamma_{n_{1}}} \\
\epsilon_{P} & =\frac{\Gamma\left(n_{1} \rightarrow P \varphi_{\mathbf{p}}\right)-\Gamma\left(n_{1} \rightarrow \bar{P} \varphi_{\mathbf{p}}^{\dagger}\right)}{\Gamma_{n_{1}}}
\end{aligned}
$$

Since the IR phenomenology will require $E$ and $P$ to be stable with comparable masses, we will simplify our discussion by considering only the asymmetry in $E$ without loss of 


\section{CHAPTER 3. UV COMPLETION: DARK ATOMS}

generality. for each number density of interest, the yields $Y_{i} \equiv n_{i} / s$ satisfy the Boltzmann equations

$$
\begin{aligned}
\frac{s H_{1}}{z} Y_{n_{1}}^{\prime} & =-\gamma_{D}\left(\frac{Y_{n_{1}}}{Y_{N_{1}}^{e q}}-1\right)+(2 \leftrightarrow 2) \\
\frac{s H_{1}}{z} Y_{\Delta E}^{\prime} & =\gamma_{D}\left[\epsilon_{E}\left(\frac{Y_{n_{1}}}{Y_{n_{1}}^{e q}}-1\right)-\frac{Y_{\Delta E}}{2 Y_{E}^{e q}} \mathcal{B}_{E}\right]+(2 \leftrightarrow 2 \text { washout }+\operatorname{transfer}(3.3 .1 \\
\frac{s H_{1}}{z} Y_{\Delta \ell}^{\prime} & =\gamma_{D}\left[\epsilon_{\ell}\left(\frac{Y_{n_{1}}}{Y_{n_{1}}^{e q}}-1\right)-\frac{Y_{\Delta \ell}}{2 Y_{\ell}^{e q}} \mathcal{B}_{\ell}\right]+(2 \leftrightarrow 2 \text { washout }+ \text { transfer } 33,3.12
\end{aligned}
$$

where ' denotes differentiation with respect to $z \equiv M_{n_{1}} / T, \Delta_{(\ell, E)}$ track the particleantiparticle asymmetries in the two sectors, $H_{1}$ is the Hubble parameter at $T=M_{n_{1}}, s$ is the total entropy density, $Y_{i}^{e q}$ are the equilibrium yields, $\mathcal{B}$ denote the branching fractions of $n_{1}$ into the corresponding channel and finally, $\gamma_{D}$ is the thermally averaged $n_{1}$ decay density

$$
\gamma_{D}=\frac{m_{n_{1}}^{3} K_{1}(z)}{\pi^{2} z} \Gamma_{n_{1}}
$$

which we have written in terms of the first modified Bessel function $K_{1}$.

In order to generate the observed scale of neutrino masses $\mathcal{O}\left(10^{-2} \mathrm{eV}\right)$ via the "SeeSaw" mechanism and the correct abundance of $\mathcal{O}(10 \mathrm{GeV})$ dark matter, we must work in the so-called "strong-strong" washout regime where both SM and dark sector partialwidths satisfy $\mathcal{B}_{\ell, E} \Gamma_{n_{1}}^{2} \gg M_{n_{1}} H\left(M_{n_{1}}\right)$. In this scenario the neutrinos remain coupled to the cosmological fluid until the $2 \leftrightarrow 2$ scattering terms (e.g. $n_{1} n_{1} \leftrightarrow \ell \ell$ ) trigger the departure from equilibrium after the neutrino number density becomes nonrelativistic. This allows $Y_{n_{1}}$ to drift from $Y_{n_{1}}^{\mathrm{eq}}$ and leave behind asymptotic particle/antiparticle asymmetries $Y_{\Delta(\ell, E)}^{\infty}$ in the $z \rightarrow \infty$ limit. 


\section{CHAPTER 3. UV COMPLETION: DARK ATOMS}
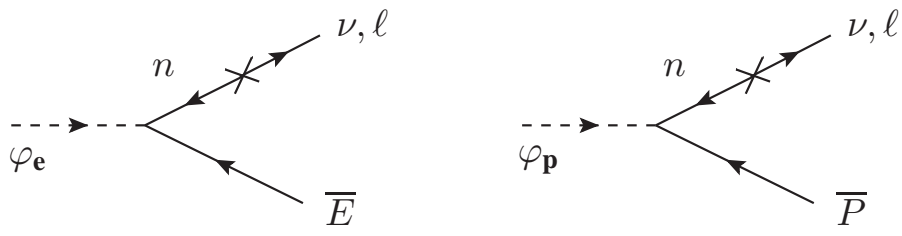

Figure 3.1: Diagrams contributing to scalar doublet decay through neutrino mass insertions. After the scalars become matter-antimatter symmetric through $\varphi_{\mathbf{e}}$ and $\varphi_{\mathbf{p}}$ number violating interactions, these decays give no net lepton number violation and the decay products annihilate into dark/visible radiation.

The Lagrangian in Eq. (3.3.6) only displays terms that exhibit a global symmetry under which $E(P)$ and $\varphi_{\mathbf{e}}\left(\varphi_{\mathbf{p}}\right)$ carry opposite charge. After electroweak symmetry breaking, the scalars $\varphi_{e, p}$ can decay to $(\ell \bar{E})$ and $(\ell \bar{P})$ final states (Figure 4.3). Since the scalars acquire particle-antiparticle excesses equivalent to their fermionic counterparts, their decays naively erase the asymptotic fermion asymmetry $Y_{\Delta E}^{\infty}$. However, the scalar potential for these fields allows terms that violate $\varphi_{\mathbf{e}}$ and $\varphi_{\mathbf{p}}$ number by two units

$$
V\left(\varphi_{\mathbf{p}}, \varphi_{\mathbf{e}}\right) \supset \kappa\left(\varphi_{\mathbf{p}} \varphi_{\mathbf{e}}\right)^{2}+\text { h.c. }
$$

and thereby initiate interconversion $\varphi_{e, p} \leftrightarrow \varphi_{e, p}^{\dagger}$. When the dark asymmetry acquires its asymptotic value at $T_{\text {asym }} \gg M_{\varphi}$, the scalars are still relativistic and the interactions in Eq. (3.3.14 equilibrate with the thermal bath to washout the scalar asymmetry before they decay out of equilibrium 1 at late times. Since there is no comparable interaction for $E$ or $P$, the resulting dark sector will only contain stable asymmetric fermions.

As with standard Leptogenesis, electroweak sphalerons generate the observed baryon

\footnotetext{
${ }^{1}$ Technically this requirement is too strong; the decay need not necessarily be out of equilibrium, but this is generically the case since the only allowed process (Figure 4.3) is suppressed by powers of $v / M_{n_{1}}$ and becomes relevant only after interconversion has frozen out.
} 


\section{CHAPTER 3. UV COMPLETION: DARK ATOMS}

number from the lepton asymmetry at high temperatures. If the Yukawa couplings $|\lambda|$ and $|y|$ are identical in magnitude and phase, then both sectors acquire the same particleantiparticle asymmetries. The ratio $\Omega_{D M} / \Omega_{B}$ will therefore have the observed value of $\simeq 6$ if the average mass in the dark sector is $\mathcal{O}(10 \mathrm{GeV})$; we will assume this to be the case throughout the remainder of this paper.

Finally, we note that in the limit where we ignore all interactions not included in Eq. (3.3.6), we can define

$$
\begin{gathered}
\sigma \equiv\left(\begin{array}{c}
\sigma_{+} \\
\sigma_{-}
\end{array}\right) \equiv \sqrt{2}\left(\begin{array}{c}
\tilde{\lambda}_{\mathbf{e}} E+\tilde{\lambda}_{\mathbf{p}} P \\
\tilde{\lambda}_{\mathbf{e}} E-\tilde{\lambda}_{\mathbf{p}} P
\end{array}\right) \\
\varphi \equiv\left(\begin{array}{c}
\varphi_{+} \\
\varphi_{-}
\end{array}\right) \equiv \sqrt{2}\left(\begin{array}{c}
\varphi_{\mathbf{e}}+\varphi_{\mathbf{p}} \\
\varphi_{\mathbf{e}}-\varphi_{\mathbf{p}}
\end{array}\right),
\end{gathered}
$$

so that Eq. (3.3.6) contains

$$
\lambda^{i} n_{i} \sigma \cdot \phi
$$

where $\tilde{\lambda}_{\mathbf{e}, \mathbf{p}} \equiv \frac{\lambda_{\mathbf{e}, \mathbf{p}}^{i}}{\lambda^{i}}$. Thus, we see explicitly that our UV theory is physically identical to that in [92], which finds robust parameter space for thermal "See-Saw" Leptogenesis with dark matter mass $m_{\chi} \simeq 10 \mathrm{GeV}$. Since the model's IR features (e.g. direct detection, structure formation) are not sensitive to the parameters in the UV Lagrangian, in the rest of the paper we take the asymmetry for granted. Furthermore, we will assume that the couplings $\lambda_{\mathbf{e}}^{i}$ and $\lambda_{\mathbf{p}}^{i}$ in Eq. (3.3.6) are such that the resulting asymmetries give equal numbers of $E$ and $P$ states at late times. 


\section{CHAPTER 3. UV COMPLETION: DARK ATOMS}

\subsubsection{Symmetry breaking and IR mass spectrum}

The scalar potential for the adjoint $\mathcal{X}$ contains

$$
V(\mathcal{X}) \supset \eta\left(\mathcal{X}^{a \dagger} \mathcal{X}^{a}\right)^{2}+\eta^{\prime} \mathcal{X}^{a \dagger} \mathcal{X}^{b} \mathcal{X}^{a \dagger} \mathcal{X}^{b}+M_{\mathcal{X}}^{2} \mathcal{X}^{a \dagger} \mathcal{X}^{a}
$$

where $a$ and $b$ are $S U(2)_{D}$ adjoint indices. While couplings to the other scalars are also allowed, we demand that $\left\langle\varphi_{\mathbf{e}}\right\rangle=\left\langle\varphi_{\mathbf{p}}\right\rangle=0$, so operators with these fields do not contribute to the minimization conditions. We have also omitted the allowed SM Higgs coupling $H^{\dagger} H \mathcal{X}^{a \dagger} \mathcal{X}^{a}$ and absorbed its vev into $M_{\mathcal{X}}$ for simplicity.

For $M_{\mathcal{X}}^{2}<0$, the adjoint scalar acquires a VEV which we can rotate into the $T_{3}$ direction without loss of generality

$$
\left\langle\mathcal{X}^{3}\right\rangle=\left\langle\mathcal{X}^{3 \dagger}\right\rangle \equiv v_{\mathcal{X}}=\sqrt{\frac{M_{\mathcal{X}}^{2}}{2\left(\eta+\eta^{\prime}\right)}} .
$$

Since $\mathcal{X}$ is an $S U(2)_{D}$ doublet with $U(1)_{X}$ charge, this implies a symmetry breaking pattern where the axial group is broken completely $S U(2)_{D} \times U(1)_{X} \rightarrow U(1)_{D}$, while the residual unbroken $U(1)_{D}$ is just the $T_{3}$ component of $S U(2)_{D}$. Henceforth, we will refer to this massless gauge field as the "dark photon."

After symmetry breaking, the fermionic doublets $E, P$ acquire masses $m_{E, P} \equiv y_{e, p} v_{\mathcal{X}}$ and residual $U(1)$ charges are determined by their $S U(2)_{D}$ isospin.

$$
E \equiv\left(\begin{array}{c}
\tilde{e} \\
e
\end{array}\right) \quad, \quad P \equiv\left(\begin{array}{c}
p \\
\tilde{p}
\end{array}\right)
$$




\section{CHAPTER 3. UV COMPLETION: DARK ATOMS}

As noted previously, gauge charges allow an EP mixing mass, which would allow atomic states to annihilate, hence we demand a $\mathcal{Z}_{2}$ symmetry to forbid this mixing and stabilize our dark matter candidate.

\subsubsection{Recombination of Multiple Atomic Species}

For sufficiently large dark couplings (e.g. $\alpha_{D} \sim 0.1$ ), aDM gives robust parameter space for early-universe recombination. The original scenario, however, assumes the minimal field content giving rise to only one species of atom: a Hydrogen-like bound state with hierarchical constituents (e.g. $m_{p} \sim 100 m_{e}$ ). In the $S U(2)_{D} \times U(1)_{X}$ model, the field content allows four distinct atomic bound states. After $\mathcal{X}$ acquires a VEV, dark "electrons" $E$ and dark "protons" $P$ generically receive different masses. Since both doublets have charge \pm 1 components $(\tilde{e}, e)$ and $(p, \tilde{p})$ under the unbroken $U(1)_{D}$ symmetry, predicting the cosmological atomic abundance requires following the evolution of 8 correlated species: $\tilde{e}, e, p, \tilde{p}, H_{e p}, H_{\tilde{e} \tilde{p}}, H_{e \tilde{e}}$ and $H_{p \tilde{p}}$. The residual $S U(2)_{D}$ global symmetry guarantees that tilded and un-tilded fields evolve in the same way, which reduces the number of independent species to five. Finally, we can reduce the number of independent equations to four if we demand that the co-moving DM number density is constant, where

$$
n_{D M}=2 n_{e}+2 n_{p}+4 N_{e p}+2 N_{e \tilde{e}}+2 N_{p \tilde{p}} .
$$




\section{CHAPTER 3. UV COMPLETION: DARK ATOMS}

If we define the following fractional yields

$$
\begin{gathered}
X_{e} n_{D M}=2 n_{e} \\
X_{p} n_{D M}=2 n_{p} \\
Y_{e p} n_{D M}=2 N_{e p} \\
Y_{p \tilde{p}} n_{D M}=2 N_{p \tilde{p}} \\
Y_{e \tilde{e}} n_{D M}=2 N_{e \tilde{e}},
\end{gathered}
$$

then Eq. (3.3.20) becomes

$$
1=X_{e}+X_{p}+2 Y_{e p}+Y_{e \tilde{e}}+Y_{p \tilde{p}}
$$

Without loss of generality we set $Y_{e \tilde{e}}=1-X_{e}-X_{p}-2 Y_{e p}-Y_{p \tilde{p}}$ and take the independent Boltzmann equations to be 2

$$
\begin{aligned}
\frac{d X_{e}}{d t} & =\frac{2}{n_{D M}}\left(C_{e p}+C_{e \tilde{e}}\right) \\
\frac{d X_{p}}{d t} & =\frac{2}{n_{D M}}\left(C_{e p}+C_{p \tilde{p}}\right) \\
\frac{d Y_{p \tilde{p}}}{d t} & =-\frac{2}{n_{D M}}\left(C_{p \tilde{p}}\right) \\
\frac{d Y_{e p}}{d t} & =-\frac{2}{n_{D M}}\left(C_{e p}\right)
\end{aligned}
$$

The collision operator $C_{i j}$ for the recombination of ions $i$ and $j$ into bound state $H_{i j}$ can be written as

$$
C_{i j}=\langle\sigma\rangle_{i j \rightarrow H_{i j} \gamma}\left(N_{i j} \frac{n_{i}^{\mathrm{eq}} n_{j}^{\mathrm{eq}}}{N_{i j}^{\mathrm{eq}}}-n_{i} n_{j}\right) ;
$$

\footnotetext{
${ }^{2}$ In the rest of this discussion we assume that $C P$-violation is negligible, i.e. the matrix elements in these Boltzmann equations are $T$-invariant.
} 


\section{CHAPTER 3. UV COMPLETION: DARK ATOMS}

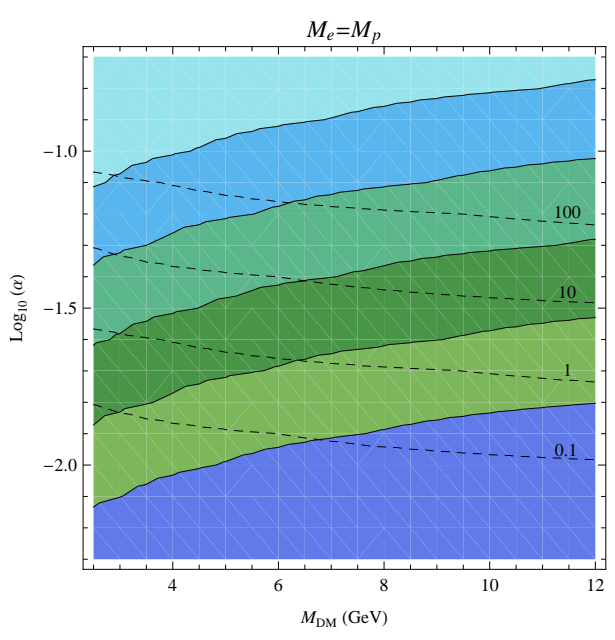

(a) Dashed lines are constant $E_{\mathrm{hf}}$ in $\mathrm{keV}$.

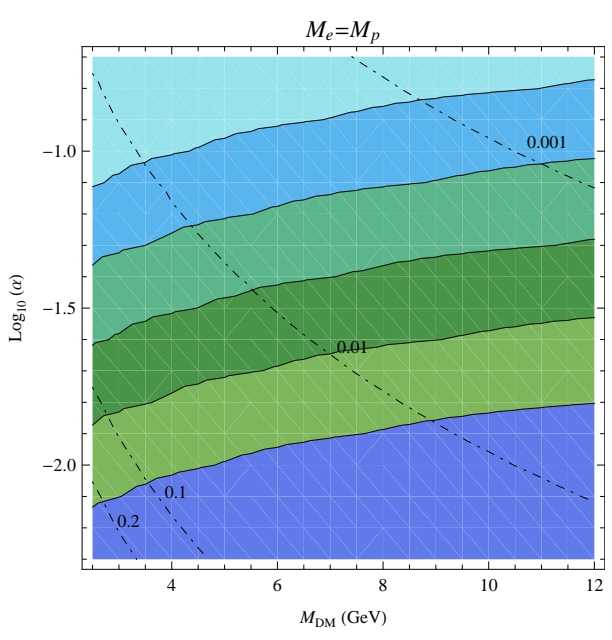

(b) Dashed lines are constant $\sigma_{\text {self }} / M_{\mathrm{DM}}$.

Figure 3.2: Solid lines show the total residual ionization. In both plots the values of the residual ionization $X_{e}$ are, from top to bottom: $>0.1,10^{-2}-10^{-1}, 10^{-3}-10^{-2}, 10^{-4}-$ $10^{-3}, 10^{-5}-10^{-4}$ and $10^{-6}-10^{-5}$. Dashed lines on the left plot indicate the hyperfine splitting in $\mathrm{keV}$, while dashed lines on the right plot indicate constant values of the ratio of the self-scattering cross section to the dark matter mass in $\mathrm{cm}^{2} / \mathrm{GeV}$. In both cases, the horizontal axis is the total mass of the dark atom.

the superscript "eq" refers to equilibrium number density and the full expression for the thermally averaged recombination cross-section can be found in our earlier paper [106] and references therein.

While the total dark matter number density depends on the abundances of all species, the "chargitronium" states $e \tilde{e}$ and $p \tilde{p}$ do not interact with ordinary matter at leading order; see Section 3.4 .2 for a detailed discussion. In Figure 3.2(a) we plot the fractional cosmological abundance of atomic states $2 Y_{e p}$ as a function of $\alpha_{D}$ and the atomic mass $m_{D M}$, including contours of constant hyperfine splitting. Observations of the bullet cluster and constraints from DM halo morphology (see Section 2.1 in [106]) demand that 


\section{CHAPTER 3. UV COMPLETION: DARK ATOMS}

$\sigma_{\text {self-scattering }} / M_{\mathrm{DM}} \lesssim 1 \mathrm{~cm}^{2} / \mathrm{g}$. The self-scattering cross sections for ion-atom and atomatom interactions are large - larger than the naïve geometric value $4 \pi a_{0}^{2}$ - because the interaction potentials are long-range, mediated by the massless dark photon. As noted in [106] and references therein, over the relevant range of interaction velocities we consider, the actual cross-sections scale as $\sigma_{\text {self-scattering }} \sim 4 \pi\left(\kappa a_{0}\right)^{2}$, where $3 \leq \kappa \leq 10$ sets the scattering length. In Figure 3.2(b) we plot the same parameter space with contours of constant $\sigma_{\text {self-scattering }} / M_{\mathrm{DM}}$. For the rest of the paper we will focus on the regions of parameter space where $2 Y_{e p} \sim \mathcal{O}(1)$ and $X_{e}+X_{p} \leq 10 \%$.

\subsubsection{Light Degrees of Freedom and the CMB}

The CMB is sensitive to the number of relativistic degrees of freedom in equilibrium with the photon gas, parameterized as the effective number of neutrinos, $N_{\nu}$

$$
\rho_{\text {rad }}=\rho_{\gamma}+\rho_{\nu}+\rho_{\gamma_{\text {dark }}}=\left[1+\zeta \frac{7}{8}\left(\frac{4}{11}\right)^{4 / 3} N_{\nu}\right] \rho_{\gamma}
$$

where $\rho_{\gamma_{\text {dark }}}$ is the radiation density due the dark photon and $\zeta \simeq 0.93$ is a parameter that corrects for neutrino/electron scattering and finite-temperature QED effects [112]. The dark photon and ordinary photon are equilibrated by dark-electron visible-electron scattering through $X$-boson exchange, which becomes inefficient when the dark electrons become non-relativistic. Their number density quickly becomes Boltzmann suppressed and the the two sectors decouple around the temperature $T_{\mathrm{dec}} \approx m_{e} / 20$, where $m_{e}$ is the mass of the dark electron. Once the dark/visible photon gasses decouple, they maintain relativistic 


\section{CHAPTER 3. UV COMPLETION: DARK ATOMS}

number densities, so any temperature difference that arises between them is due entirely to the additional freeze-out of relativistic species, which heats the visible radiation.

The dark photon's contribution to $N_{\nu}$ in the CMB depends strongly on whether the sectors decouple before or after the QCD phase transition. For dark electron masses at our scale of interest $(\sim 1 \mathrm{GeV})$, the sectors decouple around $50 \mathrm{MeV} \ll \Lambda_{Q C D}$, so the visible sector only gets reheated by standard model electron, positron and neutrino freeze-out. Between decoupling and last scattering, approximately 10 relativistic degrees of freedom freeze out in the visible sector, so the ratio of photon densities is

$$
\frac{\rho_{\gamma_{\text {dark }}}}{\rho_{\gamma}}=\left(\frac{8}{43}\right)^{4 / 3}
$$

which gives $N_{\nu} \simeq 3.4$ at last scattering in the presence of dark radiation.

\subsection{Direct Detection and Allowed Parameter}

\section{Space}

\subsubsection{Isothermal Ionic Halo}

In this section we consider the fate of dark ions that survive early-universe recombination. For simplicity, we will assume single species of dark electrons $E$ and protons $P$. In the equal mass limit, $m_{E}=m_{P}$, this assumption introduces no loss of generality and the qualitative features of this argument do not change so long as the electron and proton 


\section{CHAPTER 3. UV COMPLETION: DARK ATOMS}

masses are of the same order of magnitude. To model the cold DM, luminous disk, and bulge, we follow the discussion in [114], however our qualitative results are robust under perturbations of model input parameters and persist when we consider different CDM haloes (e.g. NFW).

In the allowed regions of aDM parameter space, atomic bound states are the dominant form of DM and both atom-atom and atom-ion scattering rates are suppressed. As such, we can safely suppose that the CDM atoms in our galaxy settle into an Einasto 3 profile [113] at late times

$$
\rho_{\text {atom }}(r)=\rho_{\odot} \exp \left\{-\frac{2}{\alpha_{e}}\left[\left(\frac{r}{a_{h}}\right)^{\alpha_{e}}-\left(\frac{r_{\odot}}{a_{h}}\right)^{\alpha_{e}}\right]\right\}
$$

where $\rho_{\odot}=0.3 \mathrm{GeV} / \mathrm{cm}^{3}$ is the local DM mass density, the Einasto index is $\alpha_{e}=0.22$, and the length scale is $a_{h}=13 k p c$. We assume that the presence of dark ions does not significantly alter the CDM profile. The luminous disk can be modeled as

$$
\rho_{d}(r, z)=\frac{\Sigma_{d}}{2 z_{d}} \exp \left(-\frac{r}{r_{d}}\right) \operatorname{sech}^{2}\left(\frac{z}{z_{d}}\right)
$$

where $(r, z)$ are cylindrical coordinates, $\Sigma=1154 M_{\odot} / p c^{2}$ is the surface density, and $r_{d}=2.54 \mathrm{kpc}\left(z_{d}=0.34\right)$ is the radial (axial) scale factor. Finally, the luminous "bulge" can be modeled as a uniform sphere centered at the galactic origin. Since this lies well within the solar radius, our model will be insensitive to the bulge profile, so the total bulge mass enclosed in radius $r$ is

$$
M_{b}(r)=M_{b}\left(\frac{r}{r_{b}}\right)^{3}
$$

\footnotetext{
${ }^{3}$ The qualitative results of this section do not change when we use the NFW profile [73] to model the dominant atomic CDM halo.
} 


\section{CHAPTER 3. UV COMPLETION: DARK ATOMS}

where $M_{b}=4.5 \times 10^{9} M_{\odot}$ and $r_{b}=1.54 \mathrm{kpc}$.

Although recombination leaves behind a global ionized fraction $X_{E}$ (see Eq. (3.2.3)), after galaxy formation, the dark-ion mass distribution inside the halo can deviate significantly from a standard profile. To investigate this phenomenon, we assume a conservative initial condition in which the ions are initially distributed in an Einsasto profile $\rho_{\text {ion }}(t=0 ; r)=X_{E} \rho_{\text {atom }}(r)$, which becomes distorted as they scatter. While this approach does not take into account the initial ionic power spectrum, it sets an upper bound on the local ionized fraction; ions encounter more friction during galactic infall and, therefore, comprise a smaller fraction of the total halo than our naive estimate $\left(\sim X_{E}\right)$ would suggest.

Following the discussions in $[33,34]$ we consider the relaxation time $\tau$ for an ion to exchange an $\mathcal{O}(1)$ fraction of its kinetic energy. The classical scattering rate is

$$
\Gamma=n_{i o n}\left(r_{\odot}\right) \sigma v\left(r_{\odot}\right)=\frac{4 \alpha_{D}^{2} n_{i o n}\left(r_{\odot}\right)}{m_{i o n}^{2} v^{3}}
$$

where $n_{\text {ion }}$ is the ion density and implicitly depends on $X_{E}$ and we have used the geometric cross section $\sigma \sim b^{2}$, where $b=2 \alpha / m_{\text {ion }} v^{2}$ is the hard-scattering impact parameter . Comparing the relaxation time, $\tau=\Gamma^{-1}$ to the galactic period, we demand that a typical ion undergoes many hard scatters during the lifetime of the galaxy

$$
\frac{\tau}{T} \simeq \frac{G^{2} M\left(r_{\odot}\right)^{2} m_{\text {ion }}^{2}}{8 \pi^{2} \alpha_{D}^{2} r_{\odot}^{3} n_{\text {ion }}\left(r_{\odot}\right)} \ll 50
$$

where $T=2 \pi r_{\odot} / v$ is the galactic period and $M(r)$ is the total (non-ionic) mass enclosed in radius $r$. In the parameter space we consider, this condition is trivially satisfied, and the 


\section{CHAPTER 3. UV COMPLETION: DARK ATOMS}

ions reach kinetic equilibrium, settling into an independent isothermal halo.

The final equilibrium temperature of the ionic halo is set by a weighted average of the initial ionic speed distribution. If we assume the ions are initially distributed virially, then by the virial and equipartition theorems, the temperature as a function of position is

$$
T(r)=\frac{G m_{i o n}}{3 r} M(r)
$$

where $M(r)$ is the total galactic mass enclosed in radius $r$. This gives an average temperature

$$
\bar{T}=\frac{1}{M_{\mathrm{G}}} \int d^{3} r \rho_{\mathrm{G}}(r) T(r)
$$

where $\rho_{\mathrm{G}}$ and $M_{\mathrm{G}}$ are the galactic mass-density and total-mass respectively. The isothermal ion number density is, therefore

$$
n_{\text {ion }}(r)=\mathcal{C} e^{-\frac{U(r)}{\bar{T}}}
$$

where $U(r)$ is the galactic gravitational potential 4 and $\mathcal{C}$ is a normalization constant $]$ set by the global ionized fraction $X_{E}$.

For benchmark values of $m_{\text {ion }}=5 \mathrm{GeV}$ and $\alpha_{D}=0.1$, the condition in Eq. (3.4.32) is trivially satisfied and the local ionized fraction becomes

$$
X_{E}\left(r_{\odot}\right) \equiv \frac{n_{\text {ion }}\left(r_{\odot}\right)}{n_{\text {ion }}\left(r_{\odot}\right)+n_{C D M}\left(r_{\odot}\right)} \sim 10^{-3} .
$$

\footnotetext{
${ }^{4}$ For $X_{E} \ll 1, U(r)$ is approximately independent of the ionized fraction so the result in Eq. 3.4.36 varies linearly with $X_{E}$.

${ }^{5}$ Since ion velocities do not vary spatially at equilibrium, the kinetic term in the Boltzmann weight has been absorbed into the normalization.
} 


\section{CHAPTER 3. UV COMPLETION: DARK ATOMS}

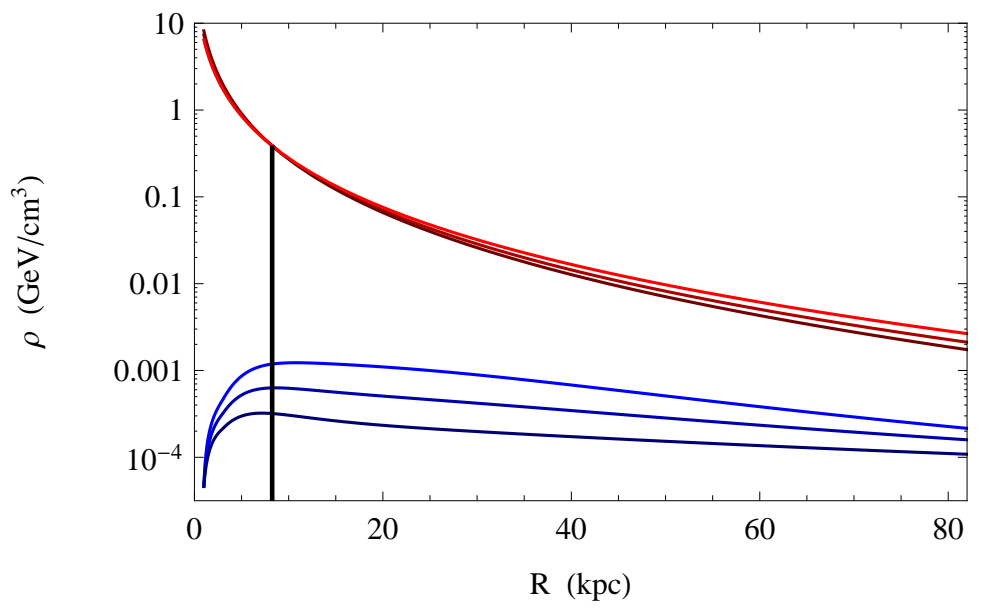

Figure 3.3: Plot of both atomic (red, higher) and ionized (blue, lower) mass densities as a function of distance from the galactic center with $m_{E}=m_{P}=5 \mathrm{GeV}$ and global ionized fraction $X_{E}=0.1$. The three lines corresponding to each distribution are calculated using best fit and $\pm \sigma$ deviations of the virial concentration parameter $C_{v}$ [114] which determines the inner slope of the Einasto profile. The vertical line at $r_{\odot}=8.25 \mathrm{kpc}$ marks the local galactic position. While $1 \sigma$ variations of the concentration parameter modifies these distributions by half an order of magnitude, their qualitative behavior is robust and the ionic density near the Sun's galactic position is generically suppressed by orders of magnitude relative to the global $X_{E}$. Similar corrections obtain unders $\pm 1 \sigma$ variation in other CDM halo inputs (e.g. galactic virial mass - local DM density); the local ionized fraction remains of order $X_{E}\left(r_{\odot}\right) \sim 10^{-3}$.

As the ion-ion scattering thermalizes, transferring heat from the core to the edge, the ions spread out away from each other to form an independent halo with farther reach than the atomic CDM distribution. This dramatic local dilution opens up a new region of parameter space previously thought to be excluded by direct detection bounds. In Figure 3.3 we plot the radial profiles for both neutral (atomic) and ionized mass densities.

Although the bullet cluster bounds allow global $X_{E} \lesssim 30 \%$ [106], to be conservative, we will only consider values around $10 \%$ for the remainder of this paper. For larger global values, the assumptions of this section are not satisfied and, furthermore, DM self-scattering 


\section{CHAPTER 3. UV COMPLETION: DARK ATOMS}

constraints seem to rule out $X_{E}>10 \%$. In any case, a dedicated numerical study is necessary to truly characterize the properties of the ionic halo. We also note that, unlike visible matter, our dark ions do not form a disk because the usual energy loss mechanisms (e.g. cooling via bremsstrahlung and molecular de-excitation) are either suppressed or unavailable.

\subsubsection{Direct Detection}

In this section we explore the $\left(M_{A}, M_{\text {Atom }}, E_{\mathrm{hf}}\right)$ parameter space in light of the positive signals at DAMA and CoGeNT, the constraints from XENON and CMDS, and recent preliminary results from CRESST [107]. We will limit ourselves to portions of parameter space where the dark matter is primarily in atomic states, though this simplification still leaves four bound states to contend with: the chargitronia $(e, \tilde{e})$ and $(p, \tilde{p})$ and the Hydrogen-like states $(e, p)$ and $(\tilde{e}, \tilde{p})$. In order to predict count rates at the various experiments we need to know both their cross-sections for scattering from standard model nuclei and their relative cosmological abundances.

First, we consider scattering rates. For a bound state of the form $(\mathcal{A}, \mathcal{B})$ the interaction Hamiltonian which allows scattering off of standard model nuclei through a dark atomic hyperfine transition has the following form

$$
\hat{H}_{\mathrm{int}} \sim Q_{\mathcal{A}} \frac{\vec{S}_{\mathcal{A}} \cdot \vec{q}}{\mu_{n \mathcal{A}}} F_{\mathcal{A}}\left(\frac{\mu_{\text {Atom }}}{m_{\mathcal{A}}} q\right)+Q_{\mathcal{B}} \frac{\vec{S}_{\mathcal{B}} \cdot \vec{q}}{\mu_{n \mathcal{B}}} F_{\mathcal{B}}\left(\frac{\mu_{\text {Atom }}}{m_{\mathcal{B}}} q\right)
$$

where the $Q_{\mathcal{I}}$ are the axial charges of the atomic constituents, $\mu_{\text {Atom }}$ is the atomic reduced 


\section{CHAPTER 3. UV COMPLETION: DARK ATOMS}

mass, the $\vec{S}_{\mathcal{I}}$ are the spin operators for the atomic constituents, $\vec{q}$ is the momentum transferred to the nucleus, the $m_{\mathcal{I}}$ are the masses of the atomic constituents, the $\mu_{n \mathcal{I}}$ are the reduced masses between nucleon and atomic constituents and the function $F_{\mathcal{I}}$ is the form factor for scattering off atomic constituent $\mathcal{I}$. The scattering rate is then proportional to the matrix element of this Hamiltonian between initial and final dark atom - nucleus states. In particular, the initial atomic state has total spin zero and the final atomic state is one of the three possible spin - 1 states. For the chargitronia, the two terms in Eq. (3.4.37) are identical, so that interaction Hamiltonian is proportional to the total spin of the chargitron. For this reason, the chargitronium atoms do not scatter from ordinary nuclei at leading order in couplings. In this regime, all the scattering rates have the same functional form, but only a fraction of the total dark matter abundance able to scatter at direct detection experiments.

Given the above argument, it is important to understand the asymptotic value of $2 Y_{e p}$, since the number of atoms able to scatter is proportional to this quantity. Furthermore, the recombination rate for bound states is proportional to

$$
\frac{\alpha^{5} m_{\text {lite }}^{3 / 2}}{\sqrt{\mu_{\text {Atom }}}}
$$

where $m_{\text {lite }}$ is the mass of the lightest atomic constituent; see our earlier work for details. This indicates that $(p, \tilde{p})$ recombines most efficiently and $(e, \tilde{e})$ combines more efficiently than the Hydrogen-like states. Note, however, that in the limit where all dark matter masses are equal, the recombination rates are equal. If we consider case where this master recombination rate leaves very few ions around, then the final abundances of each of the four 


\section{CHAPTER 3. UV COMPLETION: DARK ATOMS}

bound states will be one quarter of the total dark matter abundance and $2 Y_{e p}=1 / 2$. For the remainder of this section we work in the equal mass limit and study the direct detection parameter space as a function of three parameters $M_{X}, E_{\mathrm{hf}}$ and $M_{\mathrm{Atom}}$.

In Figure 3.4, we find the $90 \%$ and $95 \%$ favored regions for DAMA and CoGeNT in the $f_{\text {eff }}, M_{\text {DM }}$ parameter space for four different values of hyperfine splitting and with $m_{e}$ fixed to equal $m_{p}$; where $f_{\text {eff }}^{4} \equiv M_{X}^{4} /\left(2\left(g_{X} \epsilon c_{W}\right)^{2}\right)$ controls the overall size of the scattering cross-section, $g_{X}$ is the $U(1)_{X}$ coupling, and $c_{W}$ is the cosine of the weak mixing angle. We find these regions by scanning over $\chi^{2}$ per degree of freedom, based on the spectra reported in [98] and [75] respectively. In the DAMA case the $\chi^{2}$ is weighted by the reported uncertainties for each bin, whereas for CoGeNT we use Poisson statistics for the uncertainties. Figure 3.4 also includes constraint lines for XENON10 [96] and the low-threshold re-analysis of CDMS Ge [95] where we have also used Poisson statistics to define the error bars. To account for the controversy over the low-threshold behavior of $\mathcal{L}_{\text {eff }}$ bin at XENON, we plot a modified exclusion line which omits the $2-5 \mathrm{keV}$ recoil bin entirely. Any other treatment of the low-threshold behavior of XENON's detector interpolates between these two contours. The CDMS exclusion is calculated via a $\chi^{2}$ by taking the $95 \%$ confidence limit of the spectrum reported in [95] and weighting the $\chi^{2}$ with Poisson uncertainties. We find that this method adequately reproduces the "vanilla" WIMP exclusion lines reported by CDMS.

A few comments are in order. First, we see that while increasing the hyperfine splitting 


\section{CHAPTER 3. UV COMPLETION: DARK ATOMS}

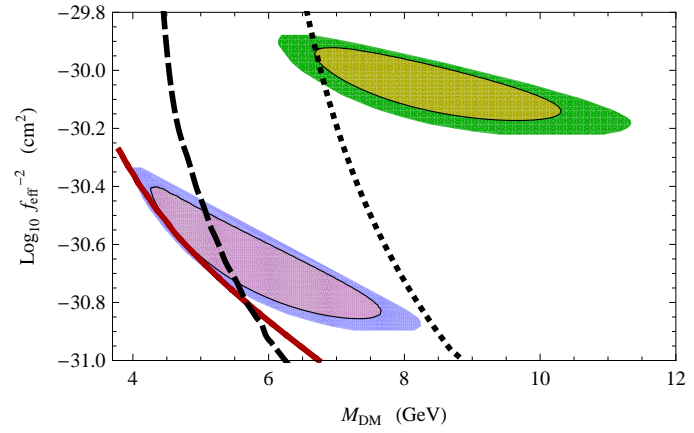

(a) $E_{h f}=5 \mathrm{keV}$

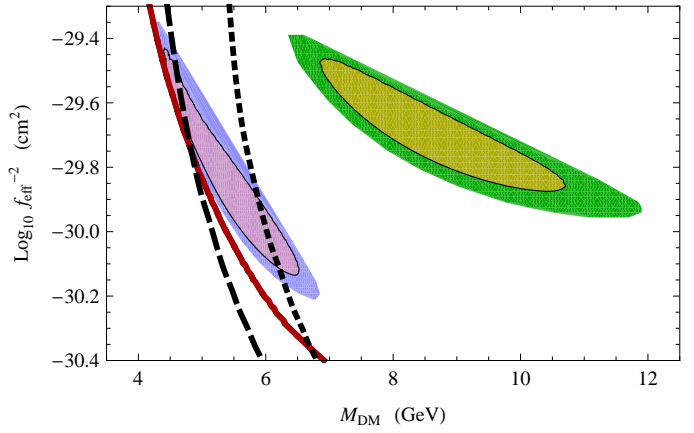

(b) $E_{h f}=15 \mathrm{keV}$

Figure 3.4: DAMA (yellow/green) and CoGeNT (purple/blue) 90\% and 95\% favored regions with CDMS-II Soudan exclusion lines (red, solid). In (a) we also include the older XENON 10 bounds $[25,96]$ using the published low-recoil sensitivity (black, dashed) and a modified efficiency which omits the lowest bin (black, dotted) to take into account the uncertainty in $\mathcal{L}_{\text {eff }}$. In (b) we use the most recent XENON 10 release [97] which is more constraining for larger hyperfine splittings. Similar considerations result in two exclusion lines using the published low-threshold sensitivity (black, dashed) and a modified efficiency (black, dotted) with a $2 \mathrm{keV}$ threshold. The CoGeNT favored region is not constrained by XENON 100 because the low-energy threshold is above the characteristic nuclear recoil energies that explain CoGeNT. Both plots assume a local dark matter density of $\rho_{d m}=0.3 \mathrm{GeV} / \mathrm{cm}^{3}$, however only $(e p)$ bound states scatter, so the effective density of scattering particles is $\rho_{d m} / 2$. Following the discussion in Section 4.1, we neglect the effects of dark-ion scattering as their local density is highly suppressed.

moves the DAMA and CoGeNT regions closer to one another - as one would expect since Germanium is a heavier nucleus than Sodium - there is no overlap between the two. As the hyperfine splitting is pushed to even higher values the CoGeNT allowed region becomes a very narrow, nearly vertical strip around $6 \mathrm{GeV}$. We note that variations in the galactic CDM Halo - especially the escape velocity - as well as known uncertainties in the DAMA quenching can improve agreement between DAMA and CoGeNT [115]. The regions plotted above are conservative in the sense that they do not take advantage of these variations. Second, note that the aDM parameter space favored by DAMA is completely ruled out by 


\section{CHAPTER 3. UV COMPLETION: DARK ATOMS}

the most recent CDMS analysis and the more constraining XENON exclusion, while the less aggressive treatment of XENON's low-threshold behavior does leave some parameter space for DAMA $]$. Third, note that increasing the hyperfine splitting does not have much of an effect on the allowed region for CoGeNT. This is reasonable, given that CDMS puts the tightest constraints on aDM and both CoGeNT and CDMS both look for Ge recoils. Dark atoms are not ruled out by the low-threshold results of CDMS or XENON, while light WIMPS apparently are, because the aDM recoil spectrum goes to zero linearly at low energies. In contrast, WIMP scattering is exponentially more likely at low recoil.

Finally, there is the matter of CRESST. Since the CRESST detector is made of Calcium

- Tungstate $\left(\mathrm{CaWO}_{4}\right)$ crystals, and the Oxygen/Tungsten recoils bands are distinguishable, CRESST is able to contemporaneously search for light DM scattering and heavy DM scattering, respectively. Preliminary results suggest that with $\mathcal{O}(550)$ kg-days of exposure CRESST sees roughly 23 events in the Oxygen band [107]. We find that the regions preferred by CoGeNT for $E_{\mathrm{hf}}=5,15 \mathrm{keV}$ are consistent at the $90 \%$ confidence level, with the count rate in Oxygen at CRESST. We find that, generically, the DAMA preferred region predicts a count rate at CRESST which is about four times too large.

\footnotetext{
${ }^{6}$ We also point out that the tension between DAMA and CoGeNT is not alleviated by ignoring the shape of the DAMA spectrum and considering only the net count rate.
} 


\section{CHAPTER 3. UV COMPLETION: DARK ATOMS}

\subsection{Discussion}

In this article we have studied the rich cosmology and parameter space of atomically bound dark matter. The abundance of dark atoms can be tied to the baryon asymmetry in which the decays of heavy sterile neutrinos generate both dark and visible sector abundances. For natural couplings to heavy neutrinos, both sectors acquire equal number densities, so the dark sector mass scale must be $\mathcal{O}(5 \mathrm{GeV})$ to reproduce the observed DM abundance. Since the gauge field that binds the dark atoms must be embedded in a non-Abelian group to avoid a Landau pole below the Planck scale, the dark matter is divided into four atomic species whose asymptotic abundances are very sensitive to the dark fine structure constant and the mass of each binding combination. The ionic species generically interact rapidly enough to maintain kinetic equilibrium and thereby form a separate, more diffuse halo than that of the cold atoms.

Our analysis has emphasized the limit where all atomic constituents have equal masses. By symmetry, the atomic species in this limit comprise equally abundant populations of "chargitronium." Because the dark atoms are light compared to the weak scale, the most significant constraints on aDM come from the low-threshold reanalyses at CDMS and XENON10/100. While there is significant tension between DAMA and CoGeNT, the parameter space favored by the CoGeNT signal - and allowed by null results - predicts a large signal at CRESST of the right order to explain the excess reported in preliminary results.

There are a number of directions for further study. The cosmology of aDM is intricate and 


\section{CHAPTER 3. UV COMPLETION: DARK ATOMS}

a full numerical study of the parameter space for both the asymmetry and recombination would be interesting. Furthermore, while it is clear that kinetic equilibrium will lead to a distinct ionic halo, the details of the aDM phase space distribution can only be determined through numerical simulations, which require knowledge of the initial power spectrum. It would also be interesting to consider the observational consequences of the ionic halo; for example, in principle there could be long range dipole-dipole interactions between galactic halos. For simplicity, the model has an exact parity that prevents dark atom decay. It would be interesting to consider soft violations of this parity and the potentially observable consequences. Finally, we have only studied the direct detection parameter space only in the case of a fully degenerate dark sector. Since both the abundance of atoms and shape of the direct detection spectrum are sensitive to the masses of the atomic constituents, the parameter space for more generic combinations is difficult to map. The possibility of better agreement between the various positive signals and null results makes a more thorough study valuable. 


\section{Chapter 4}

\section{Octotriplets}

\subsection{Introduction}

Scalar fields transforming as octets under $S U(3)_{c}$, the color group of the strong interactions, have been studied in various contexts. The simplest type of color-octet scalar is a singlet under the $S U(2)_{W}$ group of the weak interactions, and may be referred to as an 'octo-singlet'. These lead to pairs of dijet resonances at hadron colliders [116,117], and may explain [118] some deviations from the standard model predictions in the $3 b$ search performed by the CDF collaboration [119]. They also enhance the standard model Higgs boson production through gluon fusion [120]. Octo-singlets appear as composite particles due to technicolor [14] and other strong-coupling dynamics [121,122], or as elementary particles in 6-dimensional extensions of the standard model [123] and in theories with an extended color group [118, 124]. 


\section{CHAPTER 4. OCTOTRIPLETS}

Weak-doublet color-octet scalars (i.e., 'octo-doublets') differ dramatically from octosinglets because the standard model gauge symmetry allows renormalizable couplings of octo-doublets to the standard quarks [125]. Only if these couplings are highly suppressed or aligned with the standard model Yukawa couplings can the octo-doublets be light enough to be produced at the LHC [126]. An octo-doublet field includes four color-octet states: a charged particle, a neutral one and their antiparticles. The hadron collider signatures of octo-doublets have been explored in $[127,128]$.

In this paper we study 'octo-triplets': real scalar fields that transform in the adjoint representation, $(8,3,0)$, of $S U(3)_{c} \times S U(2)_{W} \times U(1)_{Y}$. An octo-triplet field includes three color-octet states: a particle of charge +1 , its antiparticle, and a neutral real particle. Akin to octo-singlets, octo-triplets are pair produced at hadron colliders through their couplings to gluons, and cannot decay into standard model fermions at renormalizable level because the Yukawa couplings are not $S U(2)_{W} \times U(1)_{Y}$ invariant. Unlike octo-singlets, octo-triplets cannot decay into gluons unless there are additional fields that generate certain dimension-7 operators.

One-loop decays of octo-triplets into a gluon and an electroweak boson are allowed, leading to interesting collider signatures involving two gluons and two electroweak bosons. We will show, however, that the rate of these decays is accidentally suppressed by two orders of magnitude compared to usual 1-loop estimates. Thus, new heavy particles could induce the dominant octo-triplet decay modes.

In the presence of some vectorlike quark of mass in the $\mathrm{TeV}$ range, the charged octo- 


\section{CHAPTER 4. OCTOTRIPLETS}

triplet may decay into a pair of standard model quarks, or into a $W$ boson and a pair of quarks if it is lighter than the top quark. This leads to a variety of collider signatures, including a dijet resonance, a $W$ boson and two softer jets. If the octo-triplet mass is in the $150-170 \mathrm{GeV}$ range, this signature may explain the $4.1 \sigma$ excess observed by the CDF Collaboration in the dijet resonance plus $W$ final state $[12,129] 1$. Some alternative explanations can be found in [118], [130]- [132]. At the LHC, octo-triplets with much larger masses $(\sim 1 \mathrm{TeV})$ may be probed in several final states.

Octo-triplets may be elementary particles (e.g., part of the 75 representation of $S U(5)$ grand unification), or may arise as composite ones, for example as fermion-antifermion bound states [122]. We treat the octo-triplets as point-like particles, which is a good approximation only when the compositeness scale is substantially higher than the octo-triplet mass.

In Section 2 we analyze the extension of the standard model by one real octo-triplet field. Section 3.1 introduces a heavy vectorlike quark which mediates octo-triplet decays into standard model quarks. Section 3.2 discusses flavor-changing processes. The Tevatron phenomenology of charged octo-triplets is explored in sections 3.3 (QCD pair production) and 3.4 (resonant pair production). The predictions for LHC are discussed in section 3.5. Our conclusions are summarized in Section 4. In the appendices we present the Feynman rules for octo-triplets, and then we compute the rates for the 3-body weak decay of the charged octo-triplet and for the 2-body 1-loop decays of color-octet scalars.

\footnotetext{
${ }^{1}$ The D0 search in the same channel [13] has a larger background and less data, so that it might not be sensitive enough to the signature proposed here.
} 


\section{CHAPTER 4. OCTOTRIPLETS}

\subsection{Octo-triplet scalar}

We consider the standard model plus an octo-triplet, $\Theta^{a \alpha}$, which is a real field of spin 0 transforming as $(8,3,0)$ under the standard $S U(3)_{c} \times S U(2)_{W} \times U(1)_{Y}$ gauge group. We use indices from the beginning of the Roman and Greek alphabets to label the $S U(3)_{c}$ and $S U(2)_{W}$ generators, respectively: $a, b, c=1, \ldots, 8$ and $\alpha, \beta, \gamma=0,1,2$.

\subsubsection{Interactions and masses}

All interactions of the octo-triplet with standard model gauge bosons are contained in the kinetic term

$$
\frac{1}{2}\left(D_{\mu} \Theta^{a \alpha}\right)\left(D^{\mu} \Theta^{a \alpha}\right)
$$

where $\mu$ is a Lorentz index and the covariant derivative is given by

$$
D_{\mu} \Theta^{a \alpha}=\partial_{\mu} \Theta^{a \alpha}+g_{s} f^{a b c} G_{\mu}^{b} \Theta^{c \alpha}+g \epsilon^{\alpha \beta \gamma} W_{\mu}^{\beta} \Theta^{a \gamma} .
$$

Here $f^{a b c}$ and $\epsilon^{\alpha \beta \gamma}$ are the totally antisymmetric tensors of the $S U(3)_{c}$ and $S U(2)_{W}$ groups, respectively, $g_{s}$ and $g$ are the $S U(3)_{c} \times S U(2)_{W}$ gauge couplings, $G_{\mu}^{a}$ is the gluon field, and $W_{\mu}^{\alpha}$ is the weak gauge field. The octo-triplet field includes three particles: an electricallyneutral color-octet real scalar $\Theta^{a 0}$, a color-octet scalar of electric charge $+1, \Theta^{a+}$, and its antiparticle $\Theta^{a-}$ :

$$
\Theta^{a \pm}=\frac{1}{\sqrt{2}}\left(\Theta^{a 1} \mp i \Theta^{a 2}\right)
$$




\section{CHAPTER 4. OCTOTRIPLETS}

When referring informally to the octo-triplet particles we use the $\Theta^{ \pm}$and $\Theta^{0}$ symbols without displaying the color index $a$.

The kinetic term (4.2.1) includes interactions of the $W$ boson with two octotriplet particles,

$$
-i g W_{\mu}^{-}\left[\left(\partial_{\mu} \Theta^{a+}\right) \Theta^{a 0}-\Theta^{a+} \partial_{\mu} \Theta^{a 0}\right]+\text { H.c. }
$$

and also with an additional gluon:

$$
2 i g g_{s} f^{a b c} G^{\mu a}\left(W_{\mu}^{+} \Theta^{b-}-W_{\mu}^{-} \Theta^{b+}\right) \Theta^{c 0}
$$

Similar interactions involve a $Z$ boson and two octo-triplet particles of the same charge, with or without an additional gluon. The interactions of like-sign octo-triplets with one or two gluons (photons) are completely specified by QCD (QED) gauge invariance. The Feynman rules for octo-triplets are given in Appendix A.

The mass of the octo-triplet arises from two terms in the Lagrangian:

$$
-\frac{1}{2}\left(M_{0}^{2}-\lambda_{H} H^{\dagger} H\right) \Theta^{a \alpha} \Theta^{a \alpha}
$$

where $\lambda_{H}$ is a real dimensionless parameter. The VEV of the standard model Higgs doublet $H$ has a value $v_{H} \simeq 174 \mathrm{GeV}$, so that the mass of the octo-triplet field is

$$
M_{\Theta}=\sqrt{M_{0}^{2}-\lambda_{H} v_{H}^{2}}
$$

We require $M_{\Theta}>0$ (i.e., $\Theta^{a \alpha}$ does not acquire a VEV) in order to preserve $S U(3)_{c}$ gauge invariance. Note that Higgs searches based on gluon fusion place a limit on $\lambda_{H}$ as a function of $M_{\Theta}[120]$. 


\section{CHAPTER 4. OCTOTRIPLETS}

The commutation relations of the Pauli matrices $\sigma^{\alpha}$ imply that other operators contributing to the octo-triplet mass, such as $\left(H^{\dagger} \sigma^{\alpha} \sigma^{\beta} H\right) \Theta^{a \alpha} \Theta^{a \beta}$, are either identical to the last one in Eq. (4.2.6) or vanish. Thus, at tree level $\Theta^{ \pm}$and $\Theta^{0}$ are degenerate states, having masses equal to $M_{\Theta}$. At one loop, the electroweak interactions break this degeneracy. The mass splitting between the charged and neutral octo-triplets is [133]

$$
\delta M \equiv M_{\Theta^{+}}-M_{\Theta^{0}} \simeq \frac{1-\cos \theta_{W}}{2 \sin ^{2} \theta_{W}} \alpha M_{W}
$$

up to corrections of order $\left(M_{W} / M_{\Theta}\right)^{2}$. We will see shortly that the octo-triplets have lifetimes much longer than the QCD scale, so that they hadronize. The lightest physical states are "octo-hadrons" given by a $\Theta^{0}$ or $\Theta^{ \pm}$bound to gluons or quark-antiquark pairs. The mass difference $\delta M$ between the lightest charged and neutral octo-hadrons is of the same sign and order of magnitude as $M_{\Theta^{+}}-M_{\Theta^{0}}$, so that $\delta M \sim 0.2 \mathrm{GeV}$.

$S U(2)_{W} \times U(1)_{Y}$ gauge-invariance forbids any renormalizable interaction of the octotriplet with standard model fermions. The most general renormalizable Lagrangian $\left(\mathcal{L}_{\Theta}\right)$ for the octo-triplet scalars is given by the kinetic term (4.2.1), the potential terms quadratic in $\Theta$ given in Eq. (4.2.6), as well as a cubic term and quartic terms:

$$
\mu_{\Theta} f^{a b c} \epsilon^{\alpha \beta \gamma} \Theta^{a \alpha} \Theta^{b \beta} \Theta^{c \gamma}-\lambda_{\Theta}\left(\Theta^{a \alpha} \Theta^{a \alpha}\right)^{2}
$$

where $\lambda_{\Theta}>0$ is a dimensionless parameter, and for simplicity we display only one quartic term. The mass parameter $\mu_{\Theta}$ may be positive or negative, but its size should not be larger

than $O\left(M_{\Theta} \lambda_{\Theta}^{-1 / 2}\right)$ in order to prevent a $\Theta \mathrm{VEV}$. The above cubic term gives the following 


\section{CHAPTER 4. OCTOTRIPLETS}

interaction among the charged and neutral octo-triplet particles:

$$
2 i \mu_{\Theta} f^{a b c} \Theta^{a+} \Theta^{b-} \Theta^{c 0}
$$

\subsubsection{Collider signals of octo-triplets}

In the $\mu_{\Theta} \rightarrow 0$ limit, the Lagrangian $\mathcal{L}_{\Theta}$ has an accidental $\mathcal{Z}_{2}$ symmetry that makes the lightest octo-triplet (i.e., $\Theta^{0}$ ) stable. The charged octo-triplet decays at tree level into $\Theta^{0}$ and an off-shell $W$ boson. Computing the 3-body width to leading order in $\delta M$ (Appendix B) we find

$$
\Gamma\left(\Theta^{ \pm} \rightarrow \Theta^{0} e^{ \pm} \nu\right) \simeq \frac{\alpha^{2}}{15 \pi \sin ^{4} \theta_{W}} \frac{(\delta M)^{5}}{M_{W}^{4}}
$$

Given the small mass splitting $\delta M \sim 0.2 \mathrm{GeV}$ [see the comment after Eq. (4.2.8)], the only other relevant decay mode is $\Theta^{ \pm} \rightarrow \Theta^{0} \mu^{ \pm} \nu$ with a decay width further phasespace suppressed compared to Eq. (4.2.11). Hence, the total tree-level width of $\Theta^{ \pm}$is $\Gamma_{\text {tree }}\left(\Theta^{ \pm}\right) \simeq 1.8 \times 10^{-16} \mathrm{GeV}$. This 3-body decay width corresponds to a decay length of $1.1 \mathrm{~cm}$.

For $\mu_{\Theta} \neq 0$, the charged octo-triplet decays into gauge bosons at one loop, with $W g$ being the only 2-body final state allowed by charge conservation. The diagrams responsible for this decay are shown in Figure 4.1. The computation of the decay width described in Appendix C gives

$$
\Gamma\left(\Theta^{ \pm} \rightarrow W^{ \pm} g\right) \simeq \frac{\alpha \alpha_{s} \mu_{\Theta}^{2}}{\pi^{3} \sin ^{2} \theta_{W} M_{\Theta}} f\left(M_{W} / M_{\Theta}\right)
$$




\section{CHAPTER 4. OCTOTRIPLETS}
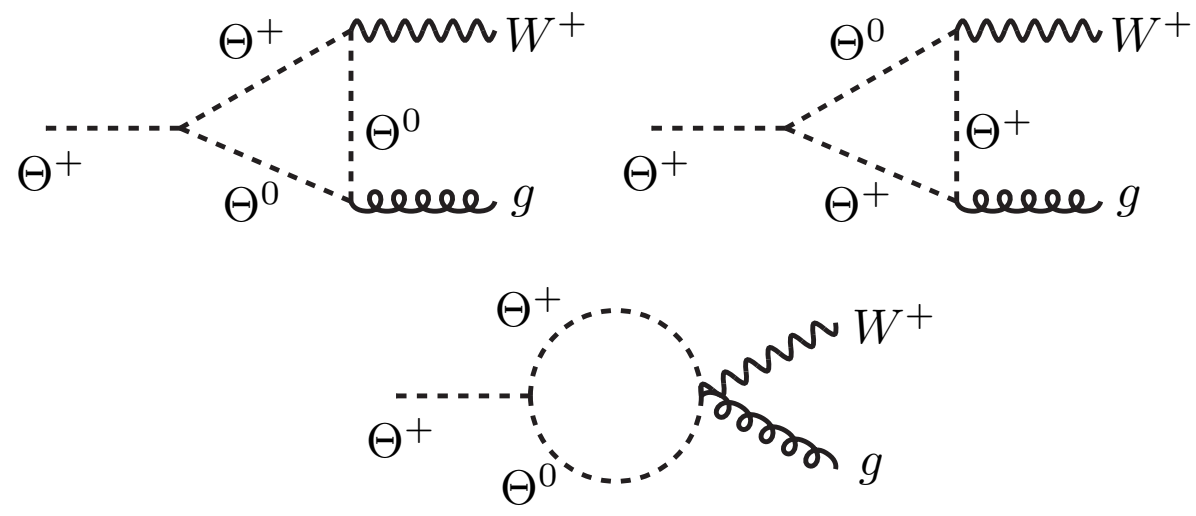

Figure 4.1: Charged octo-triplet decay to a $W$ boson and a gluon.

where the function $f(R)$ is defined in Eq. (C.8). For $M_{\Theta}$ varying between $150 \mathrm{GeV}$ and 1 $\mathrm{TeV}, f(R)$ grows from $(4.0-10.3) \times 10^{-3}$, corresponding to $\Gamma\left(\Theta^{ \pm} \rightarrow W^{ \pm} g\right)$ in the $(4.3-$ $11.2) \times 10^{-7} \mu_{\Theta}^{2} / M_{\Theta}$ range. While one might naively expect $f(R)$ to be of order one, this function is accidentally suppressed: $f(0) \propto\left(\pi^{2} / 9-1\right)^{2}$ as shown in Eq. (C.10), while for larger values of $R$, the function decreases further due to phase space suppression.

The neutral octo-triplet also decays at one loop, into a gluon and $Z$ boson or photon, with partial widths

$$
\begin{aligned}
& \Gamma\left(\Theta^{0} \rightarrow Z g\right) \simeq \frac{\alpha \alpha_{s} \mu_{\Theta}^{2}}{\pi^{3} \tan ^{2} \theta_{W} M_{\Theta}} f\left(M_{Z} / M_{\Theta}\right) \\
& \Gamma\left(\Theta^{0} \rightarrow \gamma g\right) \simeq \frac{\alpha \alpha_{s} \mu_{\Theta}^{2}}{\pi^{3} M_{\Theta}} f(0)
\end{aligned}
$$

When $M_{\Theta}$ varies between $150 \mathrm{GeV}$ and $1 \mathrm{TeV}$, the branching fraction for $\Theta^{0} \rightarrow \gamma g$ decreases from $53 \%$ to $24 \%$. The decay $\Theta^{0} \rightarrow g g$ does not occur at one loop due to $S U(2)_{W}$ invariance (this decay requires a dimension-7 operator involving two Higgs fields; further- 


\section{CHAPTER 4. OCTOTRIPLETS}

more the $\left(H^{\dagger} H\right) \Theta^{a \alpha} \Theta^{a \alpha}$ interaction does not change the $S U(2)_{W}$ indices, so that Higgs Yukawa couplings must be inserted, which is possible only at three loops).

At hadron colliders, octo-triplets are copiously pair produced due to their QCD couplings to gluons. The rate for $\Theta^{0} \Theta^{0}$ production is the same as for an octo-singlet of same mass $[117,118]$, while $\Theta^{+} \Theta^{-}$production is twice as large (additional contributions due to photon and $Z$ exchange are negligible). In Figure 4.2 we show the leading order $\Theta^{+} \Theta^{-}$ production cross section at the Tevatron and LHC, computed with MadGraph 5 [176] (with model files generated by FeynRules [177]) using the CTEQ 6 parton distribution functions [136]. The QCD corrections are not included in this plot; we expect their inclusion to shift these curves upwards by $O(50 \%)$.

Note that single octo-triplet production (through diagrams similar to those in Figure 4.1) is negligible because it is suppressed by a loop factor, the weak coupling constant, and $\left(\mu_{\Theta} / M_{\Theta}\right)^{2}$

The $\Theta^{0} \Theta^{0}$ pair leads to $(Z j)(Z j),(\gamma j)(Z j)$ and $(\gamma j)(\gamma j)$ final states, where $j$ is a gluonic jet and the parantheses indicate that the two objects form a resonance of mass $M_{\Theta}$. The $\Theta^{+} \Theta^{-}$pair leads to $\left(W^{+} j\right)\left(W^{-} j\right)$ final states, unless $\mu_{\Theta}^{2} / M_{\Theta} \lesssim O\left(10^{-9}\right) \mathrm{GeV}$ which leads to a large branching fraction for the $\Theta^{ \pm} \rightarrow \Theta^{0} e^{ \pm} \nu$ decay. This latter case gives the same final state as in $\Theta^{0} \Theta^{0}$ production because the electron and neutrino are very soft and most likely do not pass the cuts (even when $\Theta^{ \pm}$is boosted the electron is not isolated). If $\mu_{\Theta}^{2} / M_{\Theta} \simeq 4 \times 10^{-10} \mathrm{GeV}$, then the 2- and 3-body decays of $\Theta^{ \pm}$have comparable widths,

so that the $\Theta^{+} \Theta^{-}$pair leads to $(W j)(Z j)$ and $(W j)(\gamma j)$ final states, with the $(Z j)$ and 


\section{CHAPTER 4. OCTOTRIPLETS}

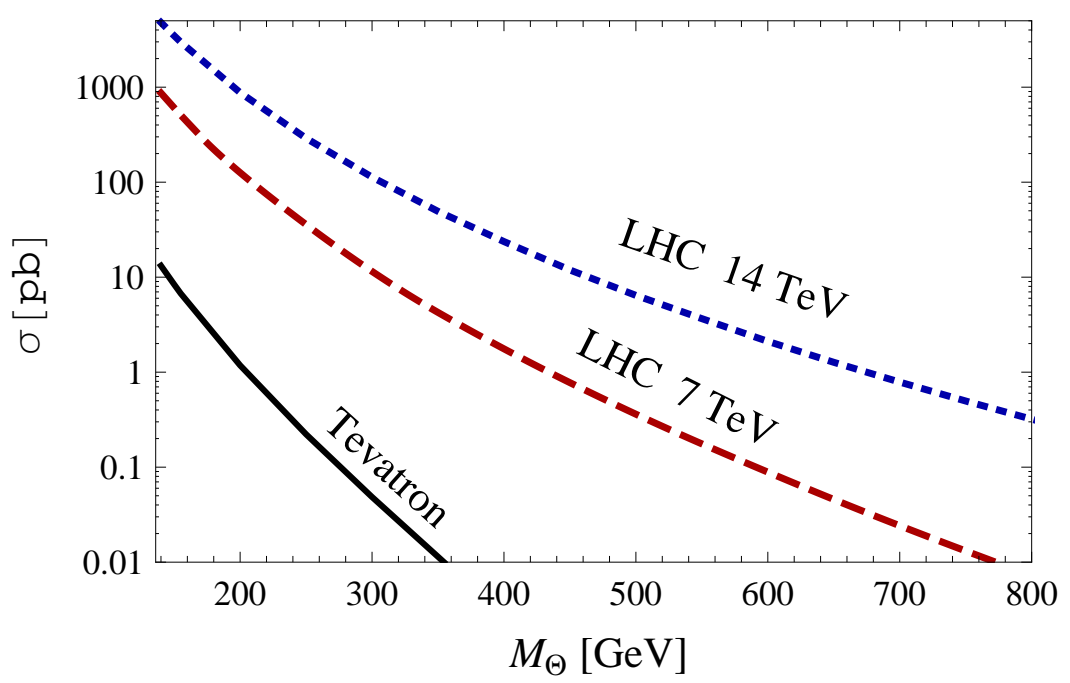

Figure 4.2: Leading order cross section for charged octo-triplet pair production at the Tevatron (black solid line) and LHC at $\sqrt{s}=7 \mathrm{TeV}$ (red dashed line) and at $\sqrt{s}=14 \mathrm{TeV}$ (blue dotted line).

$(\gamma j)$ vertices originating from displaced vertices.

Let us briefly discuss the $\left(W^{+} j\right)\left(W^{-} j\right)$ signal at the LHC, where the production cross section, Figure 4.2, can be very large. This final state is most easily identified when one $W$ decays leptonically while the other decays hadronically with an overall $W+4 j$ signature. The $W$ decay products reconstruct $W$ resonances and can thereby be isolated from the other jets in the event. These remaining jets can then be grouped alongside the known $W$ decay products and used to reconstruct pairs of octo-triplet resonances. Backgrounds to this signature include $W+$ jets production and $t \bar{t}$ pair-production, the latter of which can be substantially reduced by anti- $b$ tagging.

Besides nonresonant pair production, octo-triplet scalars may induce resonant signatures at hadron colliders because, like other long-lived colored particles $[137,138]$, they 


\section{CHAPTER 4. OCTOTRIPLETS}

form bound states. If the octo-triplet width is much less than the binding energy $E_{\mathcal{B}}$ due to gluon exchange, then bound states form before either particle decays. This is the case for all values of $M_{\Theta}$ and $\mu_{\Theta}$, as the dominant 2-body octo-triplet width given in Eq. (4.2.12) easily satisfies

$$
\Gamma\left(\Theta^{ \pm} \rightarrow W^{ \pm} g\right) \ll E_{\mathcal{B}}=\frac{9}{4} \alpha_{s}^{2} M_{\Theta}
$$

For simplicity, we consider only the formation of color-singlet bound states $\mathcal{B}$; this is the dominant channel and our qualitative conclusions apply to different color-representations of bound states.

The bound states annihilate into gauge-boson pairs before either constituent decays. Bound states of neutral octo-triplets can only annihilate through the processes $\Theta^{0} \Theta^{0} \rightarrow$ $\mathcal{B} \rightarrow g g, W^{+} W^{-}$, while the annihilation of bound states of charged octo-triplets yields a rich variety of vector boson pairs: $\Theta^{+} \Theta^{-} \rightarrow \mathcal{B} \rightarrow g g, W^{+} W^{-}, Z Z, \gamma \gamma, \gamma Z$.

Given that the bound-state effects are mainly due to gluon exchange, the production of the $\Theta^{+} \Theta^{-}$bound state is approximately equal to that of octo-singlets computed in [122]: for $M_{\Theta} \approx 150 \mathrm{GeV}$ the Tevatron cross section is $\sigma(p \bar{p} \rightarrow \mathcal{B}) \simeq O(100) \mathrm{fb}$. Since the width $\Gamma\left(\Theta^{ \pm} \rightarrow W^{ \pm} g\right)$ is orders of magnitude smaller than the main channel for bound states $\Gamma(\mathcal{B} \rightarrow g g) \simeq 0.04 \mathrm{GeV}[122]$, the annihilation dominates and yields dijet resonances with invariant mass $M_{\mathcal{B}}=2 M_{\Theta}-E_{\mathcal{B}}$. This cross section is too small to be observed at the Tevatron.

At the LHC, the bound state production cross-section can be considerably larger. For $M_{\mathcal{B}} \sim 1 \mathrm{TeV}$, the cross section is $\sigma(p p \rightarrow \mathcal{B}) \sim O(1 \mathrm{pb})[138]$ at $\sqrt{s}=14 \mathrm{TeV}$, which 


\section{CHAPTER 4. OCTOTRIPLETS}

might allow the annihilation signal to compete with the QCD background and give an observable resonance. The electroweak diboson channels are suppressed relative to $g g$, but give cleaner signals, which contribute to standard Higgs searches.

While the above discussion has been limited to the dominant color-singlet bound state, octo-triplets can also form bound states in higher color representations with exotic annihilation signatures. For instance, color-octet bound states annihilate into either $\gamma g$ or $Z g$ regardless of whether the bound state comprises charged or neutral scalars.

\subsection{Octo-triplet decays via higher-dimensional}

\section{operators}

Since the octo-triplet widths in Eqs. (4.2.11)-(4.2.12) are tiny, higher-dimensional operators induced at the $\mathrm{TeV}$ scale could lead to other decays with substantial branching fractions.

Dimension-5 operators allow the coupling of an octo-triplet to a pair of standard model quarks involving a derivative,

$$
\frac{c_{i j}}{m_{\psi}} \Theta^{a \alpha} \bar{Q}_{L}^{i} T^{a} \frac{\sigma^{\alpha}}{2} \gamma^{\mu} D_{\mu} Q_{L}^{j}+H . c .
$$

or in the presence of the Higgs doublet,

$$
\Theta^{a \alpha} \bar{Q}_{L}^{i} T^{a} \frac{\sigma^{\alpha}}{2}\left(\frac{c_{i j}^{\prime}}{m_{\psi}} \widetilde{H} u_{R}^{j}+\frac{c_{i j}^{\prime \prime}}{m_{\psi}} H d_{R}^{j}\right)
$$




\section{CHAPTER 4. OCTOTRIPLETS}

$Q_{L}^{i}, u_{R}^{j}$ and $d_{R}^{j}$ are the quark fields in the gauge eigenstate basis; $i, j=1,2,3$ label the fermion generation; $\sigma^{\alpha}$ is a Pauli matrix; $m_{\psi}$ is the mass of some heavy field that has been integrated out; and $c_{i j}, c_{i j}^{\prime}$ and $c_{i j}^{\prime \prime}$ are dimensionless coefficients. Using the field equations, one can replace the last operator, with coefficient $c_{i j}^{\prime \prime}$, by a linear transformation (involving the standard model Yukawa couplings) of the $c_{i j}$ and $c_{i j}^{\prime}$ coefficients.

\subsubsection{Octo-triplet plus a vectorlike quark}

The dimension-5 operators (4.3.1) and (4.3.2) can be induced, for example, by a heavy vectorlike quark $\Psi$ that transforms as $(3,2,1 / 6)$ under $S U(3)_{c} \times S U(2)_{W} \times U(1)_{Y}$, i.e. the same way as SM quark doublets $Q_{L}^{i}$. Renormalizable interactions of $\Psi$ with the octo-triplet,

$$
\mathcal{L}_{\Theta \Psi}=\Theta^{a \alpha} \bar{\Psi}_{R} T^{a} \frac{\sigma^{\alpha}}{2}\left(\eta_{i} Q_{L}^{i}+\eta_{\psi} \Psi_{L}\right)+\text { H.c. }
$$

and with the Higgs doublet,

$$
\mathcal{L}_{H \Psi}=\bar{\Psi}_{L}\left(\lambda_{i}^{u} H u_{R}^{i}+\lambda_{i}^{d} \widetilde{H} d_{R}^{i}\right)
$$

are allowed. Here $\eta_{i}, \eta_{\psi}, \lambda_{i}^{u}$ and $\lambda_{i}^{d}$ are dimensionless couplings and $\widetilde{H}=i \sigma^{2} H^{\dagger}$. Gaugeinvariant fermion mass terms are also allowed:

$$
-m_{\psi} \bar{\Psi}_{L} \Psi_{R}-\mu_{i} \bar{Q}_{L}^{i} \Psi_{R}+H . c .
$$

For $m_{\psi} \gg M_{\Theta}$, the $\Psi$ fermion can be integrated out, giving rise to the operators 4.3.1) through the $\mathcal{L}_{\Theta \Psi}$ interactions, and to the operators 4.3.2) through a combination of $\mathcal{L}_{\Theta \Psi}$ and $\mathcal{L}_{H \Psi}$ interactions. 


\section{CHAPTER 4. OCTOTRIPLETS}
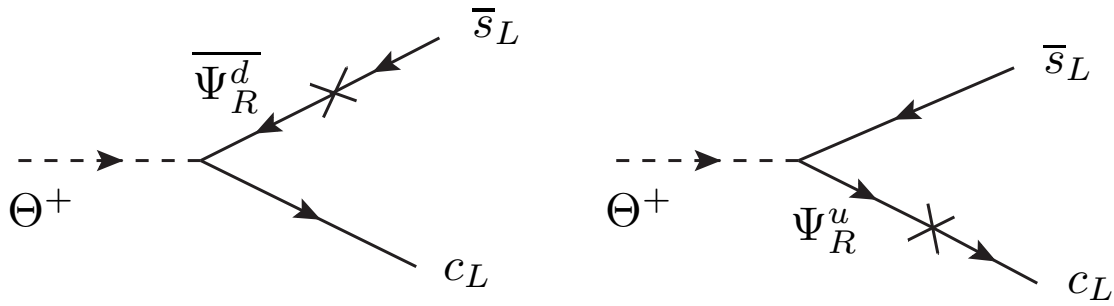

Figure 4.3: Charged octo-triplet decay to quarks in the presence of a vectorlike quark doublet $\Psi=\left(\Psi^{u}, \Psi^{d}\right)$. The mass mixing of $\Psi$ with the standard model quarks is depicted by $\times$. Similar diagrams lead to the decay of the neutral $\Theta^{0}$ scalar into quark pairs.

Let us assume for simplicity that $\lambda_{i}^{u}$ and $\lambda_{i}^{d}$ are negligible, and that the mass mixing parameters satisfy $\mu_{i} \ll m_{\psi}$. In this case the coefficients $c_{i j}$ can be computed in the mass insertion approximation:

$$
c_{i j}=-\frac{i \eta_{i}^{*} \mu_{j}}{m_{\psi}}
$$

These are the coefficients at the scale $m_{\psi}$; running down from $m_{\psi}$ to $M_{\Theta}$ may change $c_{i j}$ at $M_{\Theta}$ by an $O(1)$ factor, which we can absorb into the definition of $\eta_{i}$.

Using the quark field equations, we find that Eq. (4.3.1) contains the following interactions between $\Theta^{+}$and the mass-eigenstate quark fields $U^{i}$ and $D^{j}$ :

$$
\frac{-i}{\sqrt{2} m_{\psi}} \Theta^{a+} \bar{U}^{i} T^{a}\left[\left(C V_{K M}\right)_{i j} m_{d_{j}} P_{R}-m_{u_{i}}\left(C^{\dagger} V_{K M}\right)_{i j} P_{L}\right] D^{j}+\text { H.c. }
$$

where $V_{K M}$ is the CKM matrix, and $m_{u_{i}}, m_{d_{i}}$ are the physical masses for the quarks of the $i$ th generation. The $3 \times 3$ matrix $C$ is given by

$$
C=V_{u_{L}}^{\dagger} c V_{u_{L}}
$$

where $c$ is the matrix whose elements are given in Eq. (4.3.6), and $V_{u_{L}}$ is the matrix that 


\section{CHAPTER 4. OCTOTRIPLETS}

transforms the left-handed up-type quarks from the mass eigenstates to the gauge eigenstates, $u=V_{u_{L}} U$.

Based on interactions (4.3.7) we find that the width for the decay of the charged octotriplet into a quark pair is

$$
\Gamma\left(\Theta^{+} \rightarrow c \bar{s}\right) \simeq \frac{m_{c}^{2}+m_{s}^{2}}{64 \pi m_{\psi}^{2}}\left|C_{22}\right|^{2} M_{\Theta}
$$

where we have omitted $\mathcal{O}\left(m_{q}^{4}\right)$ terms and off-diagonal CKM elements, and have not included QCD corrections. Taking the charm quark mass $m_{c}=1.3 \mathrm{GeV}$ gives

$$
\Gamma\left(\Theta^{+} \rightarrow c \bar{s}\right) \simeq 1.3 \times 10^{-6} \mathrm{GeV}\left|C_{22}\right|^{2}\left(\frac{M_{\Theta}}{150 \mathrm{GeV}}\right)\left(\frac{1 \mathrm{TeV}}{m_{\psi}}\right)^{2}
$$

Compared with the decay into $W g$ computed in Eq. 4.2.12), the above $\Theta^{+}$decay into a pair of jets can easily dominate. For example, for $M_{\Theta}=150 \mathrm{GeV}, \mu_{\Theta}=1 \mathrm{GeV}, C_{22}=0.1$, and $m_{\psi}=1.1 \mathrm{TeV}$, we find $\Gamma\left(\Theta^{+} \rightarrow c \bar{s}\right) \simeq 3.7 \Gamma\left(\Theta^{+} \rightarrow W g\right)$.

The width for the decay into $c \bar{b}$ is sensitive to different $C_{i j}$ parameters:

$$
\frac{\Gamma\left(\Theta^{+} \rightarrow c \bar{b}\right)}{\Gamma\left(\Theta^{+} \rightarrow c \bar{s}\right)} \simeq \frac{1}{\left|C_{22}\right|^{2}}\left(\frac{m_{b}^{2}}{m_{c}^{2}}\left|C_{23}\right|^{2}+\left|C_{32}\right|^{2}\right)
$$

where $m_{b} \approx 4.2 \mathrm{GeV}$ is the $b$ quark mass.

If $M_{\Theta}>m_{t}+m_{b}$, the decay involving a top quark opens up:

$$
\Gamma\left(\Theta^{+} \rightarrow t \bar{b}\right) \simeq 2.2 \times 10^{-2} \mathrm{GeV}\left|C_{33}\right|^{2}\left(1-\frac{m_{t}^{2}}{M_{\Theta}^{2}}\right)^{2}\left(\frac{M_{\Theta}}{150 \mathrm{GeV}}\right)\left(\frac{1 \mathrm{TeV}}{m_{\psi}}\right)^{2}
$$

where we have set $m_{t}=173 \mathrm{GeV}$ and ignored $m_{b}^{2}$ terms. Due to an $m_{t}^{2} / m_{c}^{2}$ enhancement compared to Eq. (4.3.9), this decay dominates unless $\left|C_{33}\right|<10^{-2}\left|C_{22}\right|$. The decay $\Theta^{+} \rightarrow$ $t \bar{s}$ has the same width except for the $C_{33} \rightarrow C_{23}$ replacement. 


\section{CHAPTER 4. OCTOTRIPLETS}

Similar expressions give the 2-body widths for the neutral octo-triplet decaying to $c \bar{c}$, $b \bar{b}$, or top pairs if $M_{\Theta}>2 m_{t}$.

The $m_{t}^{2} / m_{c}^{2}$ enhancement in Eq. (4.3.12) is so large that even for $M_{\Theta}<m_{t}+m_{b}$ the 3-body decay through an off-shell top quark, $\Theta^{+} \rightarrow W^{+} b \bar{b}$, needs to be taken into account. Its width is

$$
\Gamma\left(\Theta^{+} \rightarrow W^{+} b \bar{b}\right)=\frac{\alpha\left|C_{33}\right|^{2} m_{t}^{4}}{64 \pi^{2} \sin ^{2} \theta_{W} m_{\psi}^{2}} \mathcal{F}\left(M_{\Theta}\right)
$$

The the function $\mathcal{F}$, of mass dimension -1 , is given by integrating the matrix element over phase space:

$$
\mathcal{F}\left(M_{\Theta}\right)=\int_{0}^{E_{0}} d \bar{E}_{\bar{b}} \int_{E_{0}-\bar{E}_{\bar{b}}}^{E_{b}^{\max }} d E_{b} \frac{E_{b}+\left(E_{0}-\bar{E}_{\bar{b}}\right)\left[\frac{2 M_{\Theta}}{M_{W}^{2}}\left(E_{0}-E_{b}\right)-1\right]}{\left(M_{\Theta}^{2}-2 M_{\Theta} \bar{E}_{\bar{b}}-m_{t}^{2}+m_{b}^{2}\right)^{2}+m_{t}^{2} \Gamma_{t}^{2}}
$$

where $E_{0}$ is the maximum energy of the $\bar{b}$ or $b$ jet,

$$
E_{0}=\frac{M_{\Theta}^{2}-M_{W}^{2}}{2 M_{\Theta}}
$$

and $E_{b}^{\max }$ is the maximum $b$ energy for a fixed $\bar{b}$ energy $\bar{E}_{\bar{b}}$,

$$
E_{b}^{\max }=\frac{E_{0}-\bar{E}_{\bar{b}}}{1-2 \bar{E}_{\bar{b}} / M_{\Theta}}
$$

In Eq. (4.3.14) we neglected $m_{b}$ everywhere (which is a good approximation for $m_{b}^{2} \ll E_{0}^{2}$ ) with the exception of the denominator where the $m_{b}^{2}$ term becomes important for $M_{\Theta}$ near the 2-body threshold, $m_{t}+m_{b}$. To cover that case we also included the top quark width, $\Gamma_{t} \approx 1.3 \mathrm{GeV}$, in the propagator. Numerically, the 3-body width can be written as

$$
\begin{aligned}
\Gamma\left(\Theta^{+} \rightarrow W^{+} b \bar{b}\right) & \simeq 2.9 \times 10^{-6} \mathrm{GeV}\left|C_{33}\right|^{2} \\
& \times \frac{\mathcal{F}\left(M_{\Theta}\right)}{\mathcal{F}(150 \mathrm{GeV})}\left(\frac{1 \mathrm{TeV}}{m_{\psi}}\right)^{2}
\end{aligned}
$$




\section{CHAPTER 4. OCTOTRIPLETS}

The ratio $\mathcal{F}\left(M_{\Theta}\right) / \mathcal{F}(150 \mathrm{GeV})$ is given by 1.51 for $M_{\Theta}=155 \mathrm{GeV}$, and by 2.28 for $M_{\Theta}=160 \mathrm{GeV}$.

It is remarkable that the above 3-body decay through a virtual top quark has a width close to that for the 2-body decay into $c \bar{s}$, given in Eq. (4.3.10). Assuming for illustration that $\left|C_{23}\right|,\left|C_{32}\right| \ll\left|C_{22}\right|=\left|C_{33}\right|$ we find that the branching fraction into $W b \bar{b}$ is 69,76 , $82 \%$ for $M_{\Theta}=150,155,160 \mathrm{GeV}$, respectively.

Finally, the decay $\Theta^{+} \rightarrow W b \bar{s}$, of width

$$
\Gamma\left(\Theta^{+} \rightarrow W^{+} b \bar{s}\right) \approx \frac{\left|C_{23}\right|^{2}}{\left|C_{33}\right|^{2}} \Gamma\left(\Theta^{+} \rightarrow W^{+} b \bar{b}\right),
$$

may also have a substantial branching fraction if the $C_{23}$ parameter is large. In that case, though, the main competing channel is likely to be $\Theta^{+} \rightarrow c \bar{b}$, as can be seen from Eq. 4.3.11.

\subsection{2 $B_{s}-\bar{B}_{s}$ mixing}

Since $\Psi$ has flavor-dependent couplings, its interactions can contribute to flavorchanging neutral processes. The largest couplings are to the $3 \mathrm{rd}$ and perhaps 2 nd generation quarks, so that we expect that the most prominent effect is in $B_{s}-\bar{B}_{s}$ meson mixing. This proceeds through the tree-level diagram in Figure 4.4. Integrating out $\Theta$ and $\Psi$ generates the effective four-Fermi operator

$$
\mathcal{L}_{B_{s}-\bar{B}_{s}}=\left(\frac{C_{23} m_{b}}{2 M_{\Theta} m_{\psi}}\right)^{2}\left(\bar{b}_{R} T^{a} s_{L}\right)^{2}+\text { H.c. }
$$




\section{CHAPTER 4. OCTOTRIPLETS}

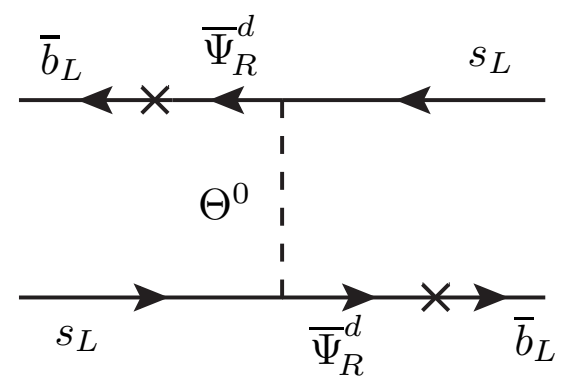

Figure 4.4: Leading contribution to $B_{s}-\bar{B}_{s}$ mixing through $\Psi$ and $\Theta$ interactions. Other diagrams differ only by the placement of $\bar{\Psi}_{R} Q_{L}$ mass insertions, and are suppressed by additional powers of $m_{s}$.

Here we have used the fermion field equations and ignored terms suppressed by factors of $m_{s} / m_{b}$

The matrix element of the Hamiltonian due to $\Theta^{0}$ exchange is

$$
\left\langle\bar{B}_{s}\left|\mathcal{H}_{\Theta}\right| B_{s}\right\rangle \simeq\left(\frac{C_{23}}{M_{\Theta} m_{\psi}}\right)^{2} M_{B_{s}}^{4} f_{B_{s}}^{2} \eta_{\mathrm{QCD}} \frac{5 B_{2}+3 B_{3}}{288}
$$

where $M_{B_{s}}$ and $f_{B_{s}}=(231 \pm 15) \mathrm{MeV}$ [139] are the $B_{s}$ meson mass and decay constant respectively; $\eta_{\mathrm{QCD}} \simeq 1.7$ is the QCD correction for the operator in Eq. (4.3.19) due to running from the scale $M_{\Theta}$ down to $M_{B_{s}}$ [140]; $B_{2} \simeq 0.80$ and $B_{3} \simeq 0.93$ are lattice "bag" parameters [141] for the singlet-singlet and octet-octet color structures arising from operator 4.3.19, respectively.

It is convenient to parametrize the contribution to $B_{s}$ mixing from $\Theta^{0}$ relative to the standard model one as

$$
\frac{\left\langle\bar{B}_{s}\left|\mathcal{H}_{S M}+\mathcal{H}_{\Theta}\right| B_{s}\right\rangle}{\left\langle\bar{B}_{s}\left|\mathcal{H}_{S M}\right| B_{s}\right\rangle} \equiv C_{B_{s}} e^{-i \phi_{s}},
$$




\section{CHAPTER 4. OCTOTRIPLETS}

where $C_{B_{s}}$ is a positive parameter and $-\pi<\phi_{s}<\pi$ is a phase. The standard model contribution can be extracted from the estimate given in [142, 143]:

$$
\left\langle\bar{B}_{s}\left|\mathcal{H}_{S M}\right| B_{s}\right\rangle \approx\left(8.0 \times 10^{-6} \mathrm{GeV}\right)^{2}(1 \pm 0.15)
$$

The $15 \%$ theoretical uncertainty shown above loosens the constraint on $C_{B_{s}}$ set by the measured $B_{s}$ mass difference: $C_{B_{s}} \approx 0.98 \pm 0.15$. Comparing Eqs. (4.3.20) and (4.3.21) we find

$$
m_{\psi}=1.1 \mathrm{TeV} \times\left|C_{23}\right|\left(\frac{150 \mathrm{GeV}}{M_{\Theta}}\right)\left(C_{B_{s}}^{2}+1-2 C_{B_{s}} \cos \phi_{s}\right)^{-1 / 4}
$$

and a less illuminating expression of $\phi_{s}$ in terms of the phase of $C_{23}$. For $M_{\Theta}=150 \mathrm{GeV}$, $C_{23}=0.2, C_{B_{s}}=0.9$, and a small CP-violating phase $\phi_{s}=-5^{\circ}$, we get $m_{\psi}=568 \mathrm{GeV}$. However, if the phase is large, as suggested by the D0 like-sign dimuon asymmetry [144], then $m_{\psi}$ is below the electroweak scale; for example $\phi_{s}=-45^{\circ}$ gives $m_{\psi}=260 \mathrm{GeV}$. Such a light vector-like quark is not ruled out. Note that the main decay mode is likely to be $\Psi^{d} \rightarrow \Theta^{+} \bar{c} \rightarrow(c \bar{s}) \bar{c}$, so that $\Psi$ pair production leads to a 6-jet final state. The CDF search [145] in a similar channel gives a lower limit on the $3 j$ resonance mass below 200 $\mathrm{GeV}$.

This model also contributes to $b \rightarrow s \gamma$ decays at the 1-loop level. Since these diagrams involve two mass insertions and suffer additional loop suppression, we expect their contributions to be small. 


\section{CHAPTER 4. OCTOTRIPLETS}

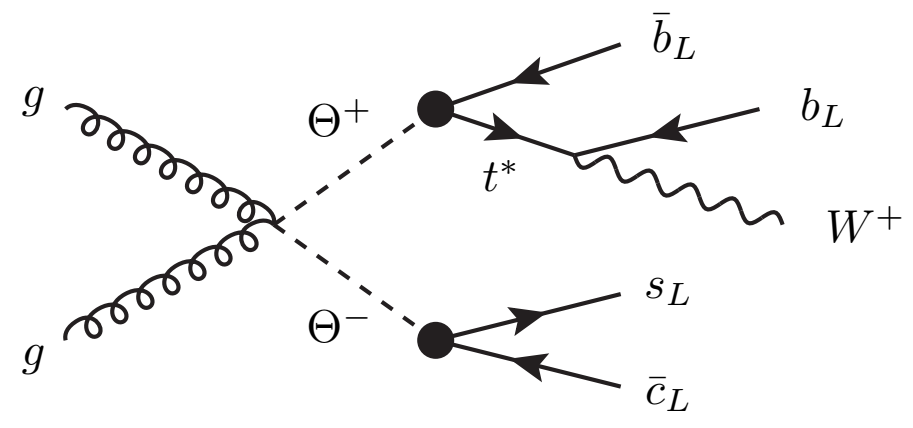

Figure 4.5: Representative diagram for pair production of charged octo-triplets through gluon fusion with a $(j j)(W b \bar{b})$ final state. The $\bullet$ symbol denotes dimension-5 operators induced by the $\Psi$ fermion. Similar diagrams lead to $4 j,\left(W^{+} b \bar{b}\right)\left(W^{-} b \bar{b}\right),\left(W^{+} b \bar{s}\right)(j j)$, or $(j b)(W b \bar{b})$ final states.

\subsubsection{Dijet resonance plus a $W$ boson at the Tevatron}

Pair production of octo-triplets, through their QCD couplings to gluons, gives rather large cross sections at the Tevatron, as shown in Figure 4.2. In the presence of the vectorlike quark $\Psi$ and assuming that the trilinear coupling $\mu_{\Theta}$ is small enough (see section 3.1), the main decay modes of $\Theta^{+}$are into a pair of jets $(c \bar{b}$ or $c \bar{s})$ and into $W b \bar{b}(W b \bar{s}$ is also possible, but at least one $b$ quark is always present due to the decay through the off-shell top quark). One of the final states (see Figure 4.5) arising from $\Theta^{+} \Theta^{-}$production is then $(j j)(W b \bar{b})$, where $j$ is any jet and the parantheses indicate a resonance at $M_{\Theta}$. The branching fractions depend on the $\left|C_{22}\right|$ and $\left|C_{33}\right|$ parameters, and are also quite sensitive to $M_{\Theta}$, as discussed at the end of section 3.1. We expect that next-to-leading order QCD corrections to this process, which affect both production and decays, increase the rate by a $K$ factor in the $1-1.5$ range. 


\section{CHAPTER 4. OCTOTRIPLETS}

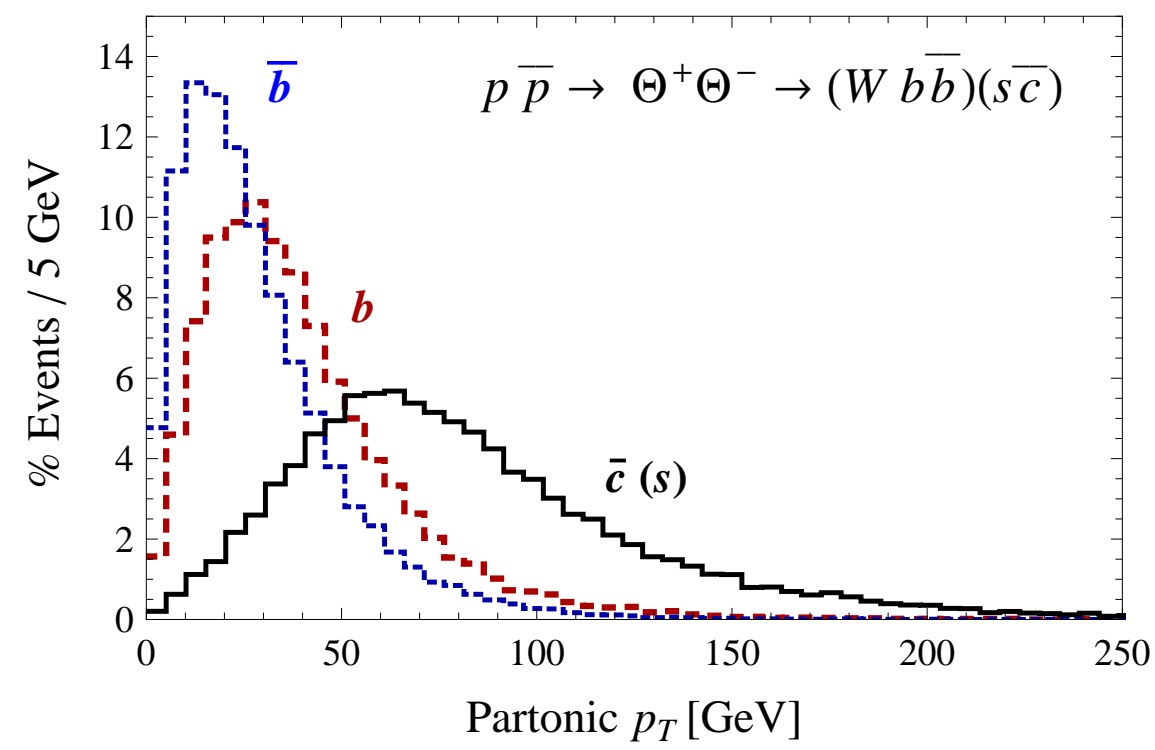

Figure 4.6: Partonic $p_{T}$ distributions for the quarks arising from the $\Theta^{+} \Theta^{-} \rightarrow(c \bar{s})\left(W^{-} b \bar{b}\right)$ process (see Figure 4.5) with $M_{\Theta}=155 \mathrm{GeV}$. The $c$ or $\bar{s}$ distribution (black solid line) peaks at higher $p_{T}$ and has a longer tail than the $b$ and $\bar{b}$ distributions (blue dotted and red dashed lines).

The two $b$ jets arising from the decay through an off-shell top quark typically have energies below $\left(M_{\Theta}-M_{W}\right) / 2-m_{b}$, so are softer than those arising from the 2-body decay, which typically have energies around $M_{\Theta} / 2$. Figure 4.6 shows the transverse momentum of each quark in the $(c \bar{s})(W b \bar{b})$ final state for $M_{\Theta}=155 \mathrm{GeV}$, computed with MadGraph 5 [176] with model files generated by FeynRules [177]. Given that the quarks from the 2-body decay have the highest $p_{T}$, the invariant mass distribution of the two leading jets from the $p \bar{p} \rightarrow \Theta^{+} \Theta^{-} \rightarrow(j j)(W b \bar{b})$ process exhibits a peak near $M_{\Theta}$.

In order to compare this signal with the CDF dijet excess [12], we generate partonic events using MadGraph 5 for the $p \bar{p} \rightarrow \Theta^{+} \Theta^{-} \rightarrow(j j)(W b \bar{b})$ process with $W \rightarrow e \nu, \mu \nu, \tau \nu$. We then use PYTHIA 6.4 [178] for hadronization and parton showering, and PGS 4 [179] 


\section{CHAPTER 4. OCTOTRIPLETS}

for detector-level effects. We impose 2 the same cuts as CDF [12]: lepton $p_{T}^{\ell}>20 \mathrm{GeV}$ and $\left|\eta^{\ell}\right|<1$, missing transverse energy $\mathbb{E}_{T}>25 \mathrm{GeV}$, transverse $W$ mass $M_{T}(W)>30 \mathrm{GeV}$, jet $p_{T}^{j}>30 \mathrm{GeV}$ and $\left|\eta^{j}\right|<2.4$, separation between jets $\left|\Delta \eta_{j j}\right|<2.5$, azimuthal separation between the missing $E_{T}$ and the leading jet $|\Delta \phi|>0.4$, and $p_{T j j} \geq 40 \mathrm{GeV}$ for the leading dijet system. The resulting dijet invariant mass $\left(m_{j j}\right)$ distribution for events with exactly 2 jets is shown in Figure 4.7 (solid red line) for $M_{\Theta}=155 \mathrm{GeV}$ and a branching fraction $\mathcal{B}_{3} \equiv \mathcal{B}\left(\Theta^{+} \rightarrow W^{+} b \bar{b}\right)=40 \%$. The rate for this process (before cuts and without including the $W \rightarrow \ell \nu$ branching fraction) is

$$
2 \mathcal{B}_{3}\left(1-\mathcal{B}_{3}\right) \sigma\left(p \bar{p} \rightarrow \Theta^{+} \Theta^{-}\right) \simeq 3.2 \mathrm{pb}
$$

where the factor of 2 takes into account the charge conjugated process with a $W^{-}$in the final state. The acceptance of the cuts is $6.2 \%$, so that in $7.3 \mathrm{fb}^{-1}$ of data there are about $470 W j j$ events due to $\Theta^{+} \Theta^{-}$production. The high-mass tail of the $m_{j j}$ distribution is mainly due to events in which the two hardest jets come from different octo-triplets.

To compare our simulated $m_{j j}$ with the CDF data shown in Figure 2 (left-side plot) of [129] we need to subtract all standard model background. The CDF Collaboration has fitted the normalization of the large CDF $W j j$ background to the data assuming a Gaussian shape for the signal. In the presence of the wider shape arising from our $\Theta^{+} \Theta^{-}$signal the $W j j$ background normalization is likely to change; increasing it by $1 \%$ gives a reasonable agreement between our $m_{j j}$ and the CDF data after background subtraction (Figure 4.7).

If the $K$ factor accounting for the QCD corrections is significantly larger than 1.0, then

\footnotetext{
${ }^{2}$ We impose cuts on the PGS output using a modified version of the Chameleon package [148].
} 


\section{CHAPTER 4. OCTOTRIPLETS}

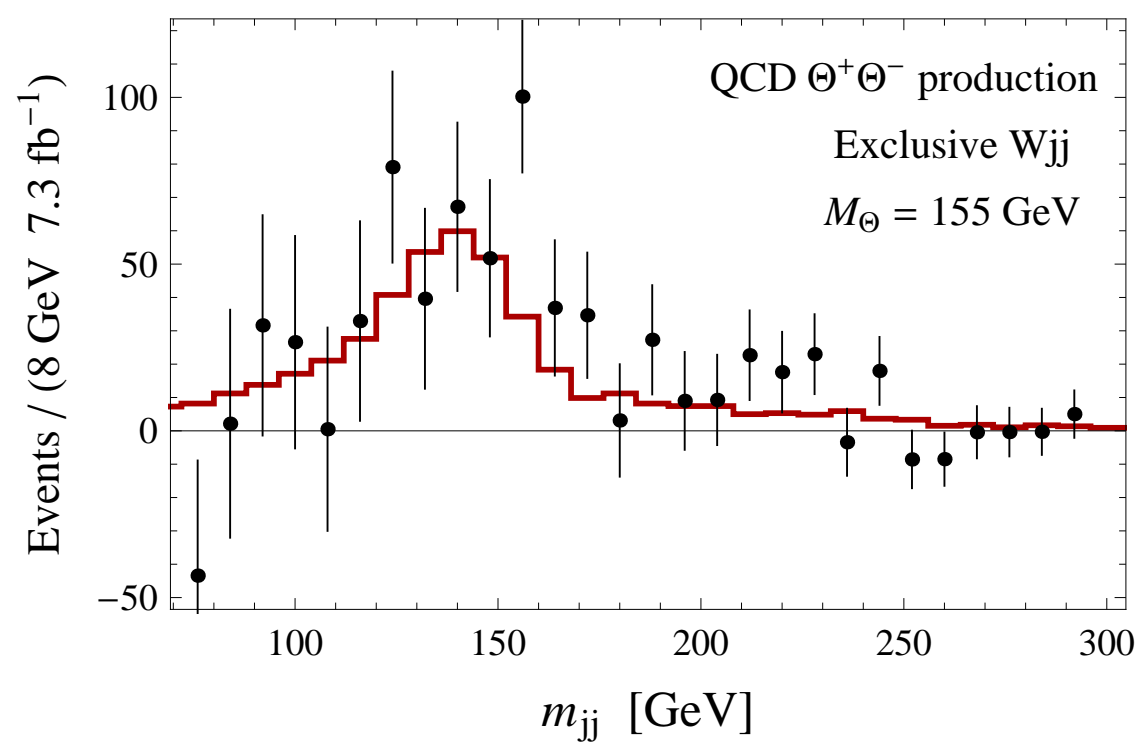

Figure 4.7: Invariant mass distribution for the leading two jets arising from the $\Theta^{+} \Theta^{-} \rightarrow$ $(j j)(W b \bar{b}) \rightarrow \ell \nu+4 j$ process, where $\ell=e, \mu$, at the Tevatron with exactly 2 jets passing the cuts. The red solid line represents events in our simulation for $\mathcal{B}\left(\Theta^{ \pm} \rightarrow W^{ \pm} b \bar{b}\right)=40 \%$ and $M_{\Theta}=155 \mathrm{GeV}$. The data points with $1 \sigma$ statistical error bars are taken from the CDF excess region (Fig. 2 of [129]) after the background (including $W W / W Z$ ) has been subtracted, with the normalization of the CDF $W j j$ background increased by $1 \%$.

$\mathcal{B}_{3}$ should be decreased while keeping the rate in Eq. 4.3.24) fixed. The highest data point, in the $152-160 \mathrm{GeV}$ bin could indicate that $M_{\Theta}$ values larger than $155 \mathrm{GeV}$ are preferred. However, the jet reconstruction performed by our PGS simulation is likely to be less efficient than the CDF reconstruction, so that a larger fraction of the hadrons is missed, reducing the jet energy. Thus, the dijet mass distribution in Figure 4.7 is likely to be artificially shifted to lower $m_{j j}$ compared to the data, implying that masses even below $155 \mathrm{GeV}$ may be acceptable. For $M_{\Theta}=150 \mathrm{GeV}$ the cross section is larger by a factor of 1.24 , so that an acceptable fit is obtained for a smaller $\mathcal{B}_{3} \approx 26 \%$.

The D0 search [13] in the same channel with $4.3 \mathrm{fb}^{-1}$ has ruled out a $1.9 \mathrm{pb}$ signal at the 95\% confidence level, based on the assumptions that the dijet resonance $X$ has a Gaussian 


\section{CHAPTER 4. OCTOTRIPLETS}

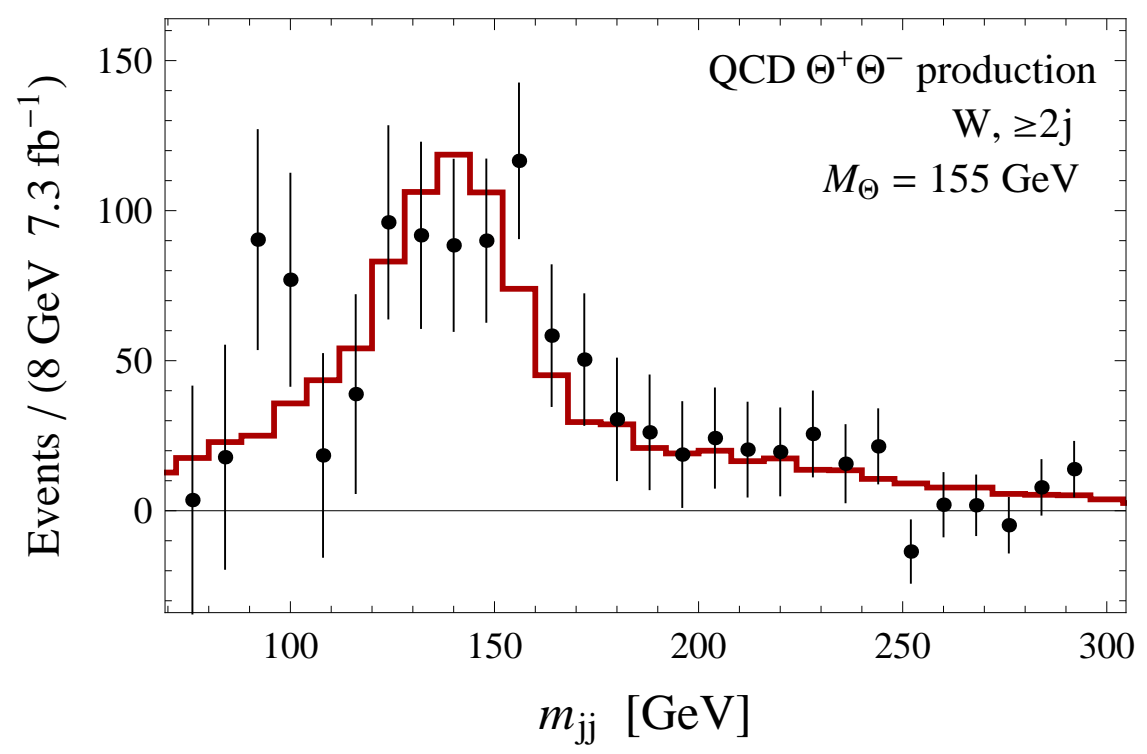

Figure 4.8: Same as Figure 4.7 except that two or more jets pass the cuts. The data points with error bars are taken from the CDF excess region (Fig. 5 of [129]) after background subtraction with the CDF $W j j$ background normalization reduced by $3 \%$.

shape with a width of $15.7 \mathrm{GeV}$ and is produced like the Higgs boson, $p \bar{p} \rightarrow W^{*} \rightarrow W X$, through a virtual $W$. Clearly, neither of these assumptions applies to our explanation for the CDF excess. The shape of our dijet invariant mass distribution is quite different than a Gaussian: it has a high tail below the peak due to final state radiation, and it has a long tail above the peak due to the two additional jets from $\Theta$ decay. The different shape is important because the fit of the background plus signal could improve significantly in the presence of our flatter signal shape compared to the pointy Gaussian. The production through $\Theta^{+} \Theta^{-}$ is also very different than through $W X$, and leads to a different acceptance. Hence, the D0 result cannot rule out our $W j j$ signal.

The requirement in the exclusive $W j j$ search [12] that exactly two jets pass the cuts rejects events arising from $\Theta^{+} \Theta^{-}$production where one of the $b$ jets has $p_{T}^{j}>30 \mathrm{GeV}$. 


\section{CHAPTER 4. OCTOTRIPLETS}

These events, however, show up in the inclusive $W j j$ search (Fig. 5 of [129]) where two or more jets pass the cuts. The normalization of the large $W j j$ background is fitted to the data independently in the exclusive and inclusive cases. The additional events mentioned above require the normalization of the CDF inclusive $W j j$ background to be reduced. Figure 4.8 shows that the QCD production of $\Theta^{+} \Theta^{-}$gives a $W+n$ jet signal with $n \geq 2$ that is consistent with the CDF data when the normalization of the CDF inclusive $W j j$ background is reduced by $3 \%$.

There are a few experimental tests of this interpretation of the CDF excess. Even though the two $b$ jets are relatively soft, the fraction of events that have a 3rd jet that passes all the cuts is large enough to allow the $b$ tagging of the 3rd hardest jet. Furthermore, the additional two $b$ jets allow the reconstruction of the full event. One complication here is that there is a large background from semileptonic $t \bar{t}$ events. Nevertheless, the signal has the property that the reconstructed $W$ boson together with the two $b$ jets form an invariant mass peak at $M_{\Theta}$, so that it can be separated from the background.

Another test is the process where both octo-triplets decay through an off-shell $t$ quark, $p \bar{p} \rightarrow \Theta^{+} \Theta^{-} \rightarrow\left(W^{+} b \bar{b}\right)\left(W^{-} b \bar{b}\right)$. The rate for this is smaller by a factor of $2\left(1 / \mathcal{B}_{3}-1\right) \approx 3$ than for the $(j j)(W b \bar{b})$ signal. Although this signal also suffers from a large $t \bar{t}$ background, it may be observable due to its relatively large rate of $\sim 1 \mathrm{pb}$ at the Tevatron.

Given that the $W$ boson in the $(j j)(W b \bar{b})$ signal originates from a decay through an off-shell top quark, there is no similar signal involving a $Z$ boson or a photon.

The process $\Theta^{+} \Theta^{-} \rightarrow(W b \bar{b})(j j)$ may affect measurements of the $t \bar{t}$ cross section. 


\section{CHAPTER 4. OCTOTRIPLETS}

However, these measurements often rely on algorithms trained specifically to find top pairs and are, thus, less sensitive to new particles that decay into similar final states. Tevatron measurements involving $b$-tags [149] may be sensitive to octo-triplet decays, but their $W$-plus-jets background normalization is fitted to the data so that they do not necessarily constrain octo-triplet decays. Furthermore, $b$-tagging efficiency decreases for softer jets such as our $b$ and $\bar{b}$ (see Figure 4.6).

Nevertheless, there are some measurements without neural networks or b-tags of the $t \bar{t}$ cross section in the lepton-plus-jets channel at the LHC [150], so these constraints must be checked more carefully. Simulating octo-triplet production with MadGraph, PYTHIA, and PGS and imposing cuts from both the ATLAS and CMS collaborations [150], we find that, for $\mathcal{B}_{3}=40 \%$ and $M_{\Theta}=155 \mathrm{GeV}$, the octo-triplet contribution to events with 3 or more jets is within the statistical error bars at $95 \%$ confidence. This approach is conservative and takes the ATLAS and CMS background simulations and extracted $t \bar{t}$ signal at face value; since these measurements are not dedicated new-physics searches, potential octotriplet decays would be bundled with the $t \bar{t}$ signal, which would make the statistical bound unrealistically constraining.

\subsubsection{Resonant production of $\Theta^{+} \Theta^{-}$}

A mass near $150 \mathrm{GeV}$ also appears in another deviation from the standard model: preliminary CDF data in the $3 b$ final state shows an excess in the invariant mass distribution of the leading two jets [119]. That deviation may arise from the $\Theta^{0} \Theta^{0} \rightarrow(b \bar{b})(b \bar{b})$ pro- 


\section{CHAPTER 4. OCTOTRIPLETS}

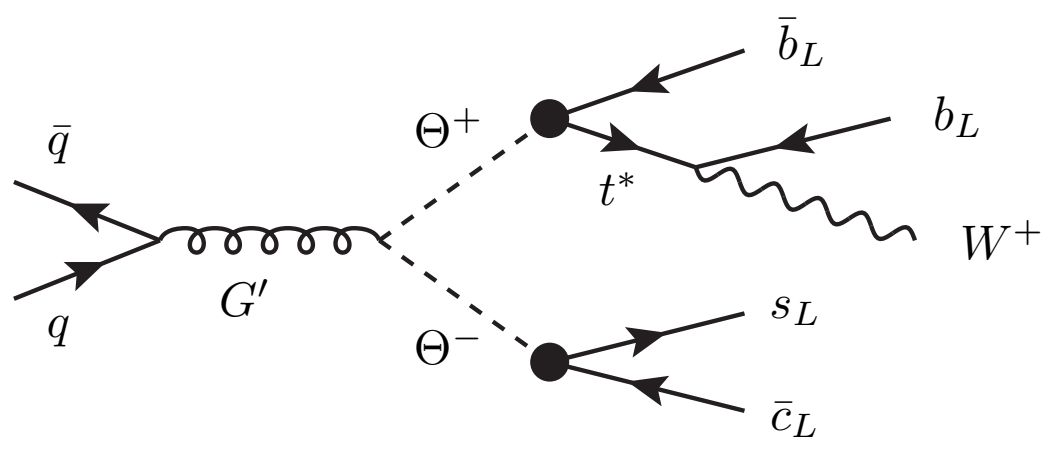

Figure 4.9: Same as Figure 4.5 except the pair of octo-triplets is resonantly produced through an $s$-channel coloron.

cess [118]. The transverse energy distributions of the jets in that case appear to favor a pair production mechanism through an $s$-channel resonance rather than through QCD. The simple renormalizable coloron model presented in [118] can be easily adapted to include the octo-triplet discussed here. It is sufficient to charge the scalar field $\Sigma$ (responsible for breaking the $S U(3) \times S U(3)$ extension of the QCD gauge group [173]) under $S U(2) \times U(1)_{Y}$, as proposed in [170]. The color-octet scalars present in the spectrum can be identified with our $\Theta^{ \pm}$and $\Theta^{0}$ (although a small mass splitting can be induced by the Higgs VEV), and they couple to the coloron field $G_{\mu}^{\prime}$ as follows

$$
\begin{aligned}
g_{s} \frac{1-\tan ^{2} \theta}{2 \tan \theta} f^{a b c} G_{\mu}^{a} & {\left[\left(\Theta^{b+} \partial^{\mu} \Theta^{c-}+H . c .\right)\right.} \\
& \left.+\Theta^{b 0} \partial^{\mu} \Theta^{c 0}\right] .
\end{aligned}
$$

Here $\tan \theta$ is a parameter in the $0.1-0.3$ range. The coloron couples to quarks proportional to $g_{s} \tan \theta$, while it couples only in pairs to gluons at renormalizable level [117]. Thus, single $G_{\mu}^{\prime}$ production proceeds entirely through quark-antiquark collisions. 


\section{CHAPTER 4. OCTOTRIPLETS}

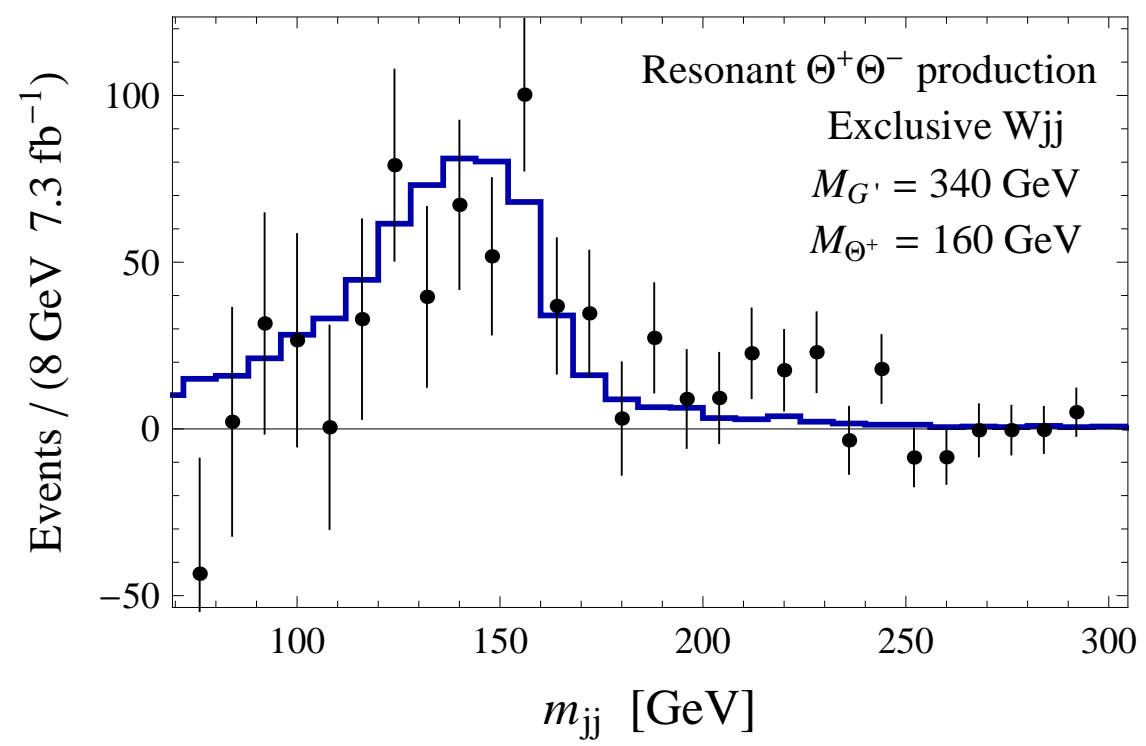

Figure 4.10: Same as Figure 4.7 except that a coloron contributes in the $s$-channel to $\Theta^{+} \Theta^{-}$ production. The blue solid line represents events in our simulation for $\mathcal{B}\left(\Theta^{ \pm} \rightarrow W^{ \pm} b \bar{b}\right)=$ $3.9 \%, M_{\Theta^{+}}=160 \mathrm{GeV}, M_{G^{\prime}}=340 \mathrm{GeV}, \tan \theta=0.15$ and $\Gamma_{G^{\prime}}=6.5 \mathrm{GeV}$.

The resonant $p \bar{p} \rightarrow G_{\mu}^{\prime} \rightarrow \Theta^{+} \Theta^{-}$production (Figure 4.9) may be an order of magnitude larger than QCD pair production [118]. This theory preserves the good agreement with the CDF data shown in Figure 4.7 provided the branching fraction $\mathcal{B}_{3}$ is decreased accordingly. The width of the coloron $\Gamma_{G^{\prime}}$ is sensitive to $\tan \theta$ and to the octet masses. Assuming that the coloron decays only into $q \bar{q}, \Theta^{+} \Theta^{-}$and $\Theta^{0} \Theta^{0}$ (additional decay channels may increase the width [118]), we find $\Gamma_{G^{\prime}}$ in the $3.2-6.5 \mathrm{GeV}$ range for $M_{\Theta^{+}}=160$ $\mathrm{GeV}, \tan \theta=0.15$, a coloron mass $M_{G^{\prime}}=340 \mathrm{GeV}$, and $M_{\Theta^{0}}$ in the $160-140 \mathrm{GeV}$ range. For this set of parameters with $\Gamma_{G^{\prime}}=6.5 \mathrm{GeV}$ we generate events as described in section 3.3. The invariant mass distribution of the leading two jets is shown in Figure 4.10 for a rate

$$
2 \mathcal{B}_{3}\left(1-\mathcal{B}_{3}\right) \sigma\left(p \bar{p} \rightarrow G^{\prime} \rightarrow \Theta^{+} \Theta^{-}\right) \simeq 3.8 \mathrm{pb}
$$




\section{CHAPTER 4. OCTOTRIPLETS}

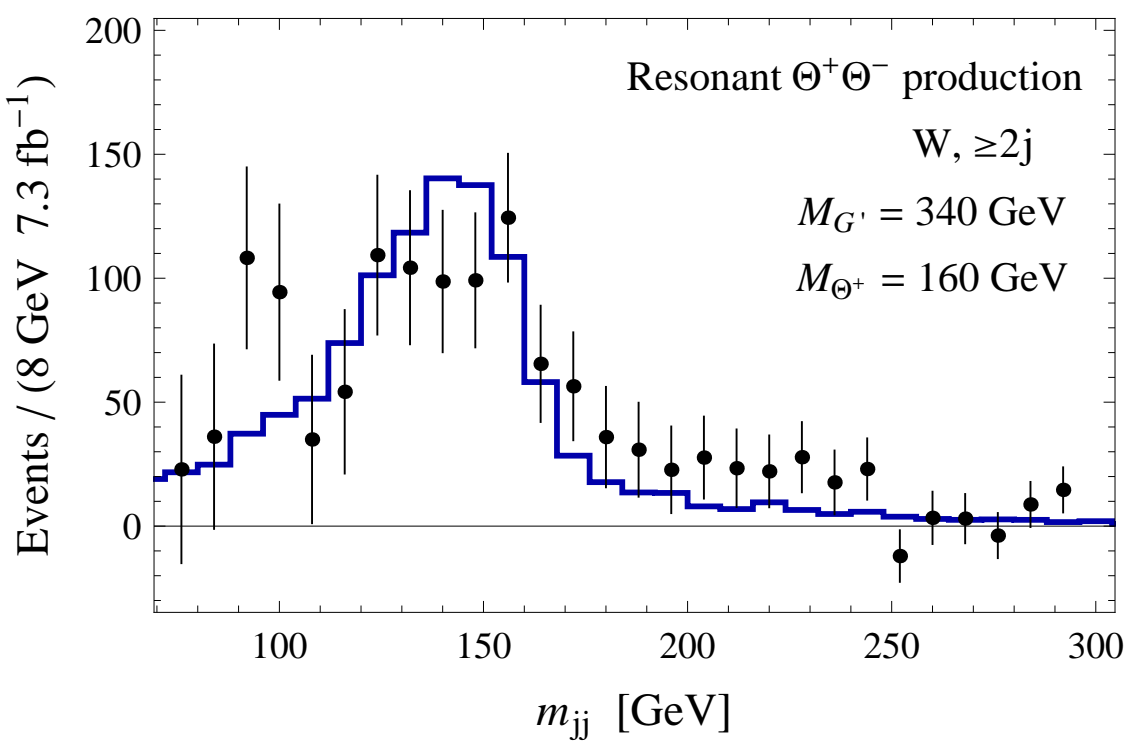

Figure 4.11: Same as Figure 4.10 except that two or more jets pass the cuts. The CDF data points are taken from Fig. 5 of [129] after background subtraction with the normalization of the CDF $W j j$ background reduced by $5 \%$.

Acceptance (without including $W$ branching fractions) is $7.3 \%$ for this process. The branching fraction inferred from the above rate is small, $\mathcal{B}_{3}=3.9 \%$, implying that the coloron $\Theta^{+} \Theta^{-}$production dominates by an order of magnitude over the QCD $\Theta^{+} \Theta^{-}$contribution. Nevertheless, we include in Figure 4.10 both production mechanisms and their interference, as well as the electroweak $\Theta^{+} \Theta^{-}$production.

Figure 4.11 shows the $m_{j j}$ distribution in the inclusive case ( $W$ plus two or more jets), with the normalization of the CDF $W j j$ background reduced by $5 \%$. The subtracted data is consistently higher than the signal in the $m_{j j} \approx 170-240 \mathrm{GeV}$ range, so one could conclude that the QCD production mechanism (see Figure 4.8) provides a better description of the CDF data. However, next-to-leading order effects are not included in these figures, and it is conceivable that they sufficiently raise the high-mass tail of the resonant production 


\section{CHAPTER 4. OCTOTRIPLETS}

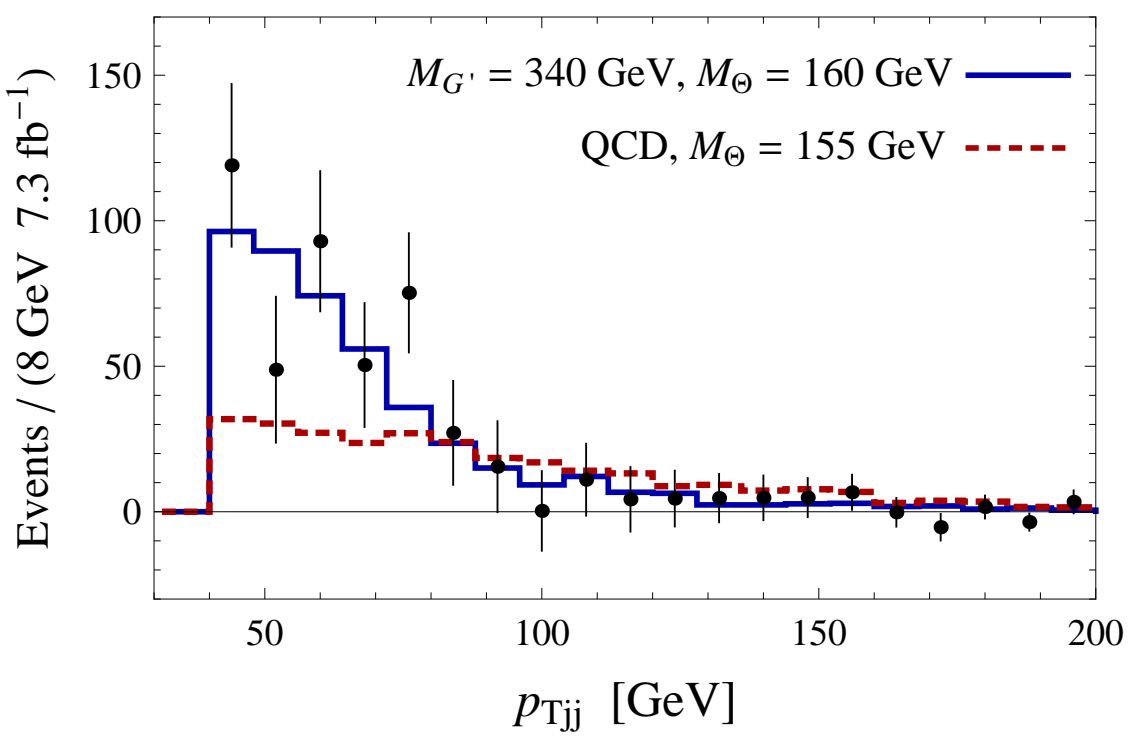

Figure 4.12: $p_{T}$ distribution of the dijet system for events satisfying $115 \mathrm{GeV} \leq m_{j j} \leq$ $175 \mathrm{GeV}$. The blue solid line is for a coloron with the same parameters as in Figure 4.10, while the red dashed line is for QCD $\Theta^{+} \Theta^{-}$production with $M_{\Theta^{+}}=155 \mathrm{GeV}$. The CDF data points with statistical error bars are taken from Fig. K9 of [153] after background subtraction consistent with Fig. 4.7

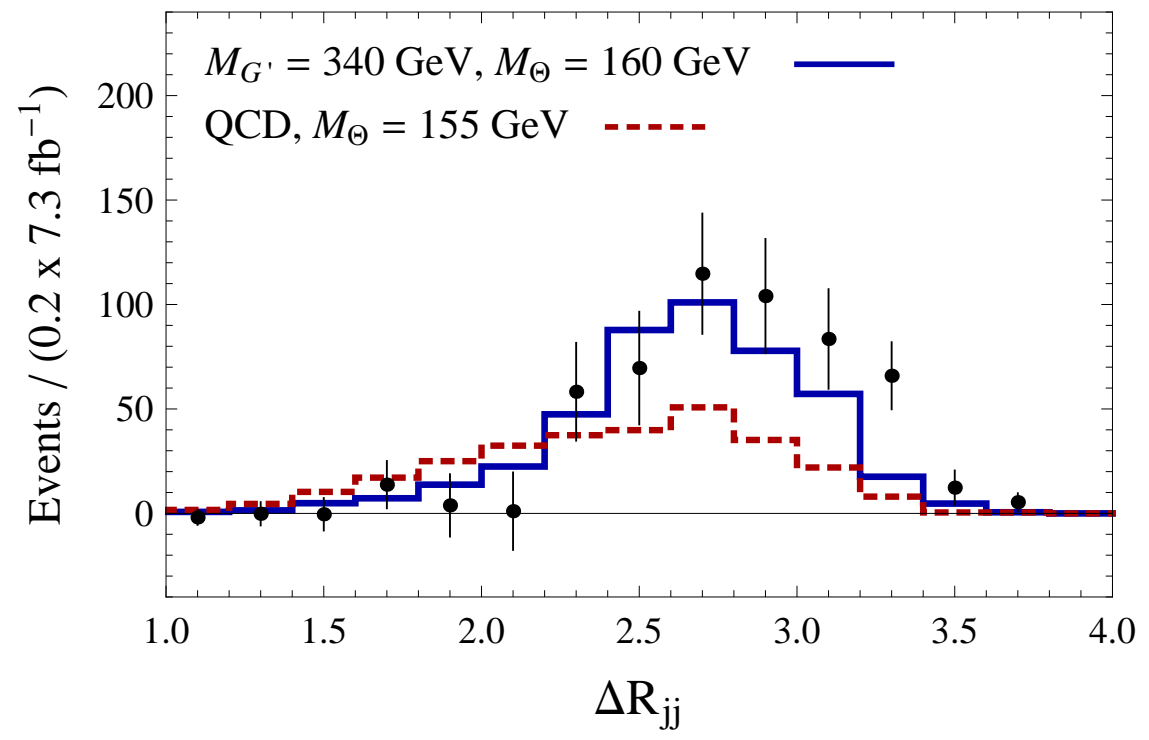

Figure 4.13: Same as Figure 4.12 for the angular separation $\Delta R_{j j}$ of the two leading jets (CDF data taken from Fig. K7 of [153]). 


\section{CHAPTER 4. OCTOTRIPLETS}

shown in Figure 4.11, Furthermore, the CDF result for the inclusive case (Fig. 5 of [129]) does not include systematic errors. We also emphasize that our detector simulation using PGS 4 [179] is only a rough approximation to the CDF full detector simulation.

A better discriminant between the resonant and QCD production mechanisms is provided by the CDF kinematic distributions [153] for the exclusive search in the $m_{j j} \approx$ $115-175 \mathrm{GeV}$ window. The transverse momentum distribution of the dijet system (Figure 4.12) shows that resonant production fits the data much better than QCD $\Theta^{+} \Theta^{-}$production. We reach the same conclusion using the $\Delta R_{j j}$ distribution of the angular separation between the two jets (Figure 4.13). Although some of the data points are not well fitted (the $p_{T j j}=72-80 \mathrm{GeV}$ bin and the $\Delta R_{j j}=3.2-3.4$ bin) by our theoretical predictions, the shapes of both the $p_{T j j}$ and $\Delta R_{j j}$ distributions are in remarkable agreement. In both Figures 4.12 and 4.13 we use the same background subtraction as in Figure 4.7, where only one background ( $W j j$ with combined electron and muon contributions) is rescaled (increased by $1 \%$ ). We expect that a fit of the standard model background plus our signal, where various background normalizations are allowed to vary, would improve the agreement between $\Theta^{+} \Theta^{-}$production and the CDF $W j j$ excess.

\subsubsection{LHC Signals}

QCD $\Theta^{+} \Theta^{-}$production, which proceeds through gluon-gluon collisions, is two orders of magnitude larger at the $7 \mathrm{TeV}$ LHC than at the Tevatron (see Figure 4.2), so that the $(j j)(W b \bar{b})$ signal discussed in section 3.3 will soon be within the reach of the CMS and 


\section{CHAPTER 4. OCTOTRIPLETS}

ATLAS experiments. Using typical parameters that explain the CDF dijet resonance, $\mathcal{B}_{3}=$ $40 \%$ and $M_{\Theta}=155 \mathrm{GeV}$, we find that the process in Figure 4.5 has a leading-order rate (before cuts) of

$$
\sigma\left(p p \rightarrow \Theta^{+} \Theta^{-} \rightarrow(j j)(\ell \nu b \bar{b})\right) \simeq 52 \mathrm{pb}
$$

where $\ell=e, \mu$. Furthermore, the $\left(W^{+} b \bar{b}\right)\left(W^{-} b \bar{b}\right)$ process also has a large rate, suppressed only by a factor of $2\left(1 / B_{3}-1\right) \approx 3$ compared to $(j j)(W b \bar{b})$, so the fully leptonic $\left(\ell^{+} \nu b \bar{b}\right)\left(\ell^{-} \bar{\nu} b \bar{b}\right)$ signal has a cross section of $3.7 \mathrm{pb}$ and will also be soon within the reach of the LHC.

In the non-minimal model (section 3.4 and Figure 4.9) where resonant $G_{\mu}^{\prime} \rightarrow \Theta^{+} \Theta^{-}$ production is the main process responsible for the CDF excess, the $(j j)(\ell \nu b \bar{b})$ rate at the $7 \mathrm{TeV}$ LHC is reduced by a factor of $\sim 5$ compared to Eq. 4.3.27), due to smaller parton distributions for quark-antiquark collissions:

$$
\sigma\left(p p \rightarrow G^{\prime} \rightarrow \Theta^{+} \Theta^{-} \rightarrow(j j)(\ell \nu b \bar{b})\right) \simeq 10 \mathrm{pb}
$$

Although QCD $\Theta^{+} \Theta^{-}$production is still present in the coloron model, explaining the CDF signal requires a 10 times smaller $\mathcal{B}_{3}$ branching fraction, which reduces the gluon initiated contribution to the $(j j)(W b \bar{b})$ signal. The smaller $\mathcal{B}_{3} \approx 3.9 \%$ also suppresses the $\left(W^{+} b \bar{b}\right)\left(W^{-} b \bar{b}\right)$ signal in this model (the rate is $\left.43 \mathrm{fb}\right)$.

While our analysis has emphasized the region around $M_{\Theta}=150 \mathrm{GeV}$, future searches could discover much heavier octo-triplets which decay into final states involving top quarks. For non-negligible values of the $C_{33}$ parameter, the processes $\Theta^{+} \Theta^{-} \rightarrow(t \bar{b})(\bar{t} b)$ 


\section{CHAPTER 4. OCTOTRIPLETS}

and $\Theta^{0} \Theta^{0} \rightarrow 4 t$ are important tests of the octo-triplet decaying through higher-dimensional operators (these final states have been studied in [128]).

\subsection{Conclusions}

We have shown that the renormalizable extension of the standard model with one octotriplet (i.e., a scalar in the adjoint representation of the standard model gauge group) involves two new parameters: the octo-triplet mass $M_{\Theta}$ and cubic self-coupling $\mu_{\Theta}$. For $\mu_{\Theta}^{2} / M_{\Theta} \gg 10^{-9} \mathrm{GeV}$ the charged octo-triplet almost always decays into $W g$ in the absence of other new particles. The rate for this 1-loop process is accidentally suppressed (see Appendix C), but the decay is prompt as long as $\mu_{\Theta}^{2} / M_{\Theta} \gtrsim 10^{-7} \mathrm{GeV}$. The neutral octo-triplet decays to $Z g$ or $\gamma g$, with widths comparable to that for $\Theta^{ \pm} \rightarrow W^{ \pm} g$. For $\mu_{\Theta} \rightarrow 0$, the main decay is a tree-level 3-body process, $\Theta^{ \pm} \rightarrow \Theta^{0} e^{ \pm} \nu$, with a displaced vertex, while $\Theta^{0}$ is stable.

At the Tevatron and the LHC, octo-triplets are produced in pairs with relatively large cross sections (see Figure 2). The main signatures are

$$
\begin{aligned}
& \Theta^{+} \Theta^{-} \rightarrow\left(W^{+} g\right)\left(W^{-} g\right), \\
& \Theta^{0} \Theta^{0} \rightarrow(Z g)(Z g),(Z g)(\gamma g),(\gamma g)(\gamma g) .
\end{aligned}
$$

The rates for these processes suggest that Tevatron experiments can be sensitive to $M_{\Theta}$ up to a few hundred $\mathrm{GeV}$, and $\mathrm{LHC}$ experiments to $M_{\Theta}$ above $1 \mathrm{TeV}$; however, more precise sensitivity estimates require detailed studies of the backgrounds. 


\section{CHAPTER 4. OCTOTRIPLETS}

Since octo-triplets have very small widths, decays through higher-dimensional operators may compete with the 1-loop processes. Operators of the type $\Theta \bar{Q} \not \partial Q$ may be induced by a heavy vectorlike quark, and lead to the $\Theta^{+} \rightarrow t \bar{b}$ decay for $M_{\Theta} \gtrsim 175 \mathrm{GeV}$. For a lighter octo-triplet, there is competition between the 2-body decays $\Theta^{+} \rightarrow c \bar{s}$ or $c \bar{b}$ and the 3-body decays $\Theta^{+} \rightarrow t^{*} \bar{b} \rightarrow W b \bar{b}$ or $t^{*} \bar{s} \rightarrow W b \bar{s}$ through an off-shell top quark. The neutral octo-triplet decays mainly to $b \bar{b}, c \bar{c}, W^{+} b \bar{c}$ and $W^{-} \bar{b} c$ for $M_{\Theta} \lesssim m_{t}+m_{c}$, to $t \bar{c}$ and $\bar{t} c$ for larger masses below $2 m_{t}$, and to $t \bar{t}$ for masses above $2 m_{t}$. For a range of parameters, the branching fractions for these decays are larger than the ones into a gluon plus an electroweak boson mentioned above. The collider signatures for $M_{\Theta} \lesssim 175 \mathrm{GeV}$ then include

$$
\begin{aligned}
& \Theta^{+} \Theta^{-} \rightarrow(j c)(W b \bar{b}),(b c)(W b \bar{b}),\left(W^{+} b \bar{b}\right)\left(W^{-} b \bar{b}\right),(j c)(j \bar{c}),(j b)(j \bar{b}), \ldots, \\
& \Theta^{0} \Theta^{0} \rightarrow(b \bar{b})(b \bar{b}),(b \bar{b})(c \bar{c}),(b \bar{b})(W b c), \ldots
\end{aligned}
$$

For a heavier octo-triplet the signatures are mainly $(t \bar{b})(\bar{t} b)$ and $(t \bar{c})(\bar{t} c)$, while above 350 $\mathrm{GeV}$ the $4 t$ final state also opens up.

Signatures of pair production followed by one octo-triplet decaying through higherdimensional operators and the other decaying into a gluon and an electroweak gauge boson at one loop are also possible. These include $\Theta^{+} \Theta^{-} \rightarrow(W g)(j c), \Theta^{0} \Theta^{0} \rightarrow(Z g)(c \bar{c})$ or $(\gamma g)(c \bar{c})$ and similar processes involving $b$ quarks (or $t$ quarks if kinematically allowed).

Some of the final states mentioned above, namely $(j c)(W b \bar{b}),(j c)(W b j),(j c)(W g)$, may be relevant to the CDF excess [12] in the dijet resonance plus $W$ search. In the case where $\Theta^{+}$decays mostly into $c \bar{s}$ and $W^{+} b \bar{b}$, so that the process is $p \bar{p} \rightarrow \Theta^{+} \Theta^{-} \rightarrow$ 


\section{CHAPTER 4. OCTOTRIPLETS}

$(c j)(W b \bar{b})$, we have shown that the $b$ jets are substantially softer than the jets originating from the $\Theta^{+} \rightarrow c \bar{s}$ decay. Events where these $b$ jets do not pass the CDF cuts could explain the dijet resonance plus $W$ signal if $\Theta$ has a mass in the $150-170 \mathrm{GeV}$ range.

We have compared two production mechanisms of charged octo-triplet pairs: through the QCD couplings to gluons (these are always present due to gauge invariance), and through an $s$-channel resonance (we have focused on a coloron, but a $Z^{\prime}$ coupled to octotriplets would not be very different). Both mechanisms are consistent with the CDF excess in the dijet invariant mass distribution when exactly two jets are required to pass the cuts. In the inclusive case (two or more jets pass the cuts), QCD $\Theta^{+} \Theta^{-}$production fits the CDF data more precisely than resonant production. However, this difference is not conclusive given that the low tail of the resonant production compared to the background-subtracted data may be due to systematic errors in the standard model background, and may also be corrected by a fit of the background (with several free normalizations, as usual) plus coloron signal. Other kinematic distributions obtained by CDF [153] can differentiate various models more effectively. We have shown that the shapes of the transverse momentum distribution for the dijet system $\left(p_{T j j}\right)$ and of the angular separation distribution for the two leading jets $\left(\Delta R_{j j}\right)$ agree rather well with the resonant mechanism while being quite different than the predictions of QCD $\Theta^{+} \Theta^{-}$production.

It is intriguing that almost the same mass $(\sim 150 \mathrm{GeV})$ appears in another deviation from the standard model, namely the $3 b \mathrm{CDF}$ search [119], which could be attributed to the $\Theta^{0} \Theta^{0} \rightarrow(b \bar{b})(b \bar{b})$ process [118]. Resonant production through a coloron also agrees better 


\section{CHAPTER 4. OCTOTRIPLETS}

to various kinematic distributions in that case.

The interpretation of the dijet plus $W$ signal in terms of an octo-triplet decaying via an off-shell top quark can be tested by the $b$-tagging of the third jet, or by the reconstruction of the $W b \bar{b}$ peak at the same mass as the dijet peak.

At the $7 \mathrm{TeV}$ LHC, if octo-triplet production is through the QCD couplings to gluons, then the dijet-plus- $W$ signal has a large cross section (52 pb for $M_{\Theta^{+}}=155 \mathrm{GeV}$ ) because it is dominated by gluon fusion. In the case of dominant production through an $s$-channel resonance coupled to $q \bar{q}$ like the coloron, the LHC signal is reduced to $\sim 10 \mathrm{pb}$.

If the couplings of the vectorlike quark are complex, then tree-level $\Theta^{0}$ exchange in-

duces $\mathrm{CP}$ violation in $B_{s}-\bar{B}_{s}$ mixing. For a vectorlike quark mass of a few hundred $\mathrm{GeV}$ (which is allowed because its main decay is into three jets), this effect can be large enough to produce a significant part of the like-sign dimuon asymmetry observed by the D0 Collaboration [144].

We note that similar final states can also arise from a fermiophobic octo-doublet field. However, this has nontrivial couplings to the standard model Higgs doublet, so the mass splitting between charged and neutral components may be large. By contrast, the tiny mass splitting between charged and neutral octo-triplets suppresses the tree-level 3-body decays. Furthermore, the neutral octo-doublet decays into gluons, while $S U(2)_{W}$ symmetry forbids pure gluonic decays of the neutral octo-triplet. 


\section{CHAPTER 4. OCTOTRIPLETS}

\section{Appendix A: Feynman Rules}

The Feynman rules for octo-triplets, derived from Eqs. (4.2.1)-(4.2.5), are given by:
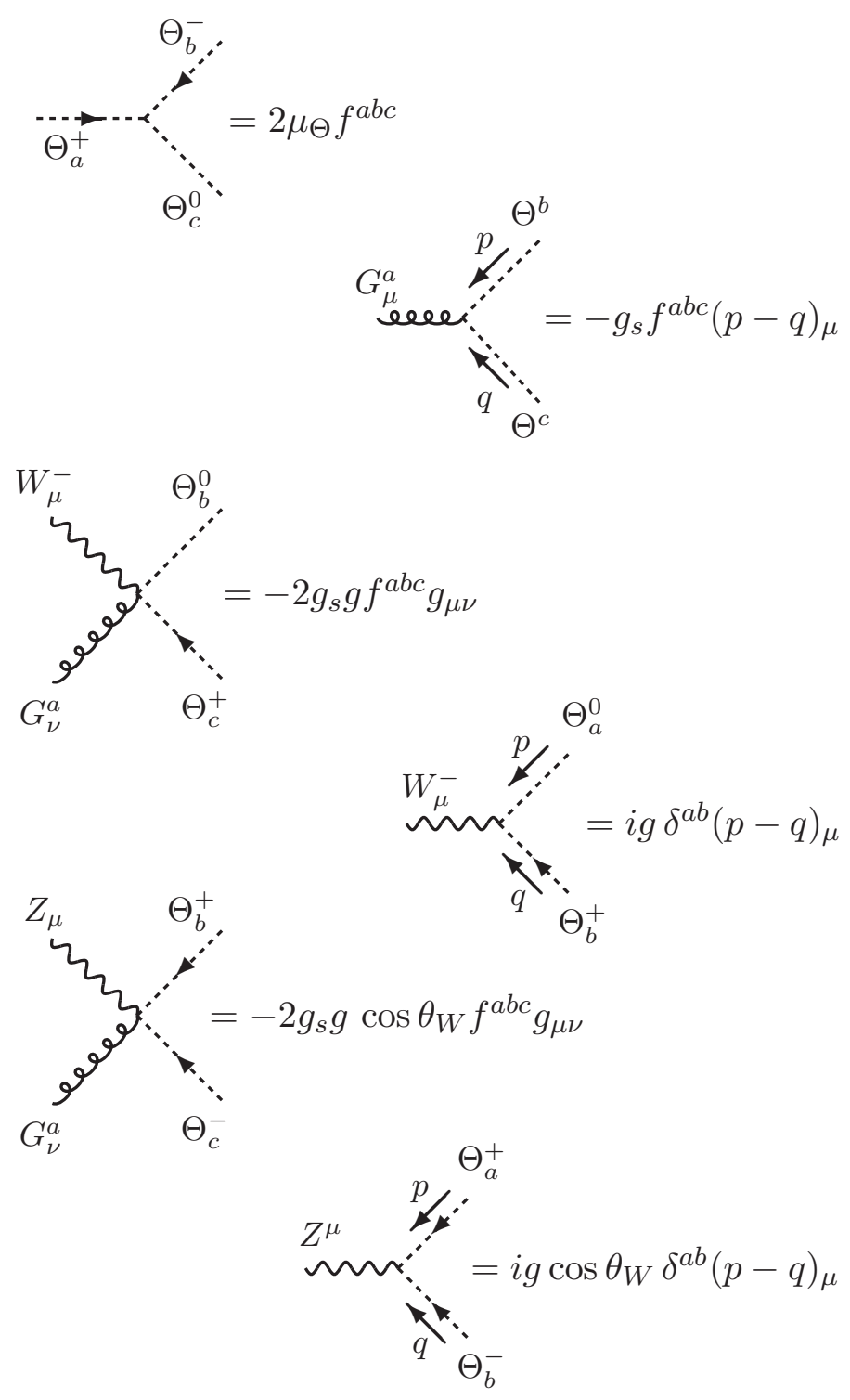

The Feynman rules involving photons are identical to those involving a $Z$ boson shown above but with the replacement $g \cos \theta_{W} \rightarrow e$. 


\section{Appendix B: Tree-level octo-triplet decay}

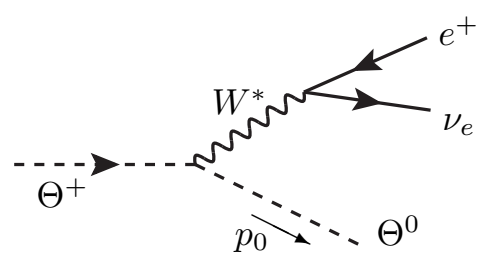

Figure 4.14: 3-body decay of the charged octo-triplet scalar through an off-shell $W$ boson.

In this appendix we compute width for the 3-body decay of the charged octo-triplet through an off-shell $W$, as shown in Figure 4.14. We define $p_{e}, p_{\nu}$, and $p_{0}$ to be the outgoing momenta for $e, \nu$ and $\Theta^{0}$ respectively. Using the Feynman rule from Appendix A, the amplitude for this process is

$$
\mathcal{M} \simeq \frac{\sqrt{2} g^{2}}{M_{W}^{2}-2 p_{e} \cdot p_{\nu}} \bar{u}\left(p_{e}\right) \not{ }_{0} P_{L} v\left(p_{\nu}\right)
$$

where we have used the $e, \nu$ equations of motion in the massless lepton limit. Squaring the amplitude and summing over helicities we find

$$
\overline{|\mathcal{M}|^{2}}=4 g^{4} \frac{2\left(p_{e} \cdot p_{0}\right)\left(p_{\nu} \cdot p_{0}\right)-p_{e} \cdot p_{\nu} M_{\Theta^{0}}^{2}}{\left(M_{W}^{2}-2 p_{e} \cdot p_{\nu}\right)^{2}} .
$$

The decay width in the $\Theta^{+}$rest frame is then given by

$$
\begin{array}{r}
\Gamma\left(\Theta^{+} \rightarrow \Theta^{0} e^{+} \nu\right)=\frac{g^{4} M_{\Theta^{+}}}{16 \pi^{3}} \int_{0}^{\varepsilon} d E_{\nu} \int_{\varepsilon-E_{\nu}}^{E_{e}^{\max }} d E_{e} \\
\times \frac{M_{\Theta^{+}}\left(\varepsilon-E_{\nu}-E_{e}\right)+2 E_{\nu} E_{e}}{\left[M_{W}^{2}-2 M_{\Theta^{+}}\left(\varepsilon-E_{\nu}-E_{e}\right)\right]^{2}}
\end{array}
$$

where $\varepsilon$ is the maximum lepton energy,

$$
\varepsilon=\frac{M_{\Theta^{+}}^{2}-M_{\Theta^{0}}^{2}}{2 M_{\Theta^{+}}},
$$




\section{CHAPTER 4. OCTOTRIPLETS}

and $E_{e}^{\max }$ is the maximum positron energy for a fixed neutrino energy $E_{\nu}$,

$$
E_{e}^{\max }=\frac{\varepsilon-E_{\nu}}{1-2 E_{\nu} / M_{\Theta^{+}}}
$$

The integrals in Eq. (B.3) can be performed analytically, with the result

$$
\Gamma\left(\Theta^{+} \rightarrow \Theta^{0} e^{+} \nu\right)=\frac{\alpha^{2} M_{W}^{4}}{16 \pi \sin ^{4} \theta_{W} M_{\Theta^{+}}^{3}} \mathcal{G}\left(\frac{\varepsilon}{M_{\Theta^{+}}}, \frac{2 M_{\Theta^{+}}^{2}}{M_{W}^{2}}\right)
$$

where we introduced a function

$$
\begin{aligned}
\mathcal{G}(x, r) & =-\xi(1+r-r x) \ln \left(1-x+r x^{2}+\xi x\right) \\
& -\frac{1}{2}\left[\left(\frac{1}{2}-x\right) r^{2}+\xi^{2}-\xi(1+r-r x)\right] \ln (1-2 x) \\
& +\frac{r^{3} x^{3}}{3}+\frac{3}{2} r^{2} x(1-x)+r x
\end{aligned}
$$

with

$$
\xi \equiv \xi(x, r)=\left[2 r+(1-r x)^{2}\right]^{1 / 2}
$$

Interestingly, the expansion of $r^{-4} \mathcal{G}(x, r)$ for $|x|<1$ starts at $x^{5}$, and the leading $r$ dependent term arises even later, at $x^{7}$ :

$$
\mathcal{G}(x, r)=\frac{r^{4} x^{5}}{15}\left[1+x+\frac{2}{7}(4-r) x^{2}\right]+O\left(x^{8}\right)
$$

Translating this expansion into a power series in $\delta M \equiv M_{\Theta^{+}}-M_{\Theta^{0}}$ we find that the exact tree-level width of Eq. (B.6) is given, up to corrections of order $(\delta M)^{8}$, by

$$
\begin{aligned}
\Gamma\left(\Theta^{+} \rightarrow \Theta^{0} e^{+} \nu\right) & \simeq \frac{\alpha^{2}(\delta M)^{5}}{15 \pi \sin ^{4} \theta_{W} M_{W}^{4}}\left[1-\frac{3(\delta M)}{2 M_{\Theta^{+}}}\right. \\
+ & \left.\frac{4}{7}\left(\frac{9}{8}-\frac{M_{\Theta^{+}}^{2}}{M_{W}^{2}}\right)\left(\frac{\delta M}{M_{\Theta^{+}}}\right)^{2}\right]
\end{aligned}
$$




\section{Appendix C: One-loop decay of a scalar octet into}

\section{gauge bosons}

In this Appendix we compute the width for a color-octet scalar decaying to a gluon and a (massive or massless) vector boson $V_{\mu}$, which proceeds through scalar 1-loop diagrams like those of Figure 4.1. In particular, this computation applies to the process $\Theta^{ \pm} \rightarrow$ $W^{ \pm} g, \Theta^{0} \rightarrow \gamma g$ or $Z g$

We label the $V_{\mu}$ and gluon 4-momenta (polarizations) by $p_{1}\left(\epsilon_{1}\right)$ and $p_{2}\left(\epsilon_{2}\right)$, respectively. Since the gluon is always transversely polarized $\left(\epsilon_{2} \cdot p_{2}=0\right)$, angular momentum conservation demands that the other vector also be transverse, so $\epsilon_{1} \cdot p_{1}=0$. Given that the contraction $\epsilon_{\mu \nu \rho \sigma} p_{1}^{\mu} \epsilon_{1}^{\nu} p_{2}^{\rho} \epsilon_{2}^{\sigma}$, cannot arise from scalar triangle diagrams, the amplitude contains only two terms: $\epsilon_{1} \cdot \epsilon_{2}$ and $\left(\epsilon_{1} \cdot p_{2}\right)\left(\epsilon_{2} \cdot p_{1}\right)$. Furthermore, by the Ward-Takahashi identity the amplitude vanishes upon replacing $\epsilon_{2}$ with $p_{2}$, so that the most general amplitude due to scalar loops is given by

$$
\mathcal{M}=\frac{\mu_{\Theta} g_{s} \tilde{g}}{\pi^{2}} \mathcal{C}\left(\frac{1}{2} \epsilon_{1} \cdot \epsilon_{2}-\frac{\left(\epsilon_{1} \cdot p_{2}\right) \epsilon_{2} \cdot p_{1}}{M_{\Theta}^{2}-M_{V}^{2}}\right)
$$

where $\mu_{\Theta}$ is the scalar trilinear coupling, $\tilde{g}$ is the scalar-vector gauge coupling, $M_{V}$ and $M_{\Theta}$ are the $V_{\mu}$ and scalar masses. The dimensionless coefficient $\mathcal{C}$ is the only quantity that needs to be computed from loop integrals.

We now compute the coefficient $\mathcal{C}$ for the process $\Theta^{+} \rightarrow W^{+} g$ by evaluating the 1-loop 


\section{CHAPTER 4. OCTOTRIPLETS}

diagrams

$$
\begin{aligned}
\mathcal{M}=\int \frac{d^{4} k}{(2 \pi)^{4}} \frac{48 i \mu_{\Theta} g_{s} g}{\left(k^{2}-M_{\varphi}^{2}\right)\left[\left(p_{1}+p_{2}+k\right)^{2}-M_{\varphi}^{2}\right]} \\
\times\left(\frac{1}{4} \epsilon_{1} \cdot \epsilon_{2}-\frac{\epsilon_{1} \cdot\left(p_{2}+k\right)\left(\epsilon_{2} \cdot k\right)}{\left(p_{2}+k\right)^{2}-M_{\varphi}^{2}}\right),
\end{aligned}
$$

where $k$ is the loop 4-momentum and $M_{\varphi}$ is the mass of the scalars running in the loop. The logarithmic divergences from the three diagrams cancel, and $\mathcal{C}$ in Eq. (C.1) can be written as a Feynman parameter integral

$$
\mathcal{C}=\int_{0}^{1} d x \int_{0}^{1-x} d y \frac{-3\left(1-R^{2}\right) x y}{1-x y-R^{2} x(1-x-y)}
$$

with $R \equiv M_{W} / M_{\varphi}$. Here we have assumed $M_{\varphi}=M_{\Theta}$, as is the case for the octo-triplet (see Figure4.1). After integration over $y$ we obtain

$$
\mathcal{C}=\frac{-3}{2\left(1-R^{2}\right)}\left[\frac{\pi^{2}}{9}-1+R^{2}\left(\frac{\pi}{\sqrt{3}}-1\right)+2 J(R)\right]
$$

where we have defined

$$
J(R)=\int_{0}^{1} d x\left(\frac{1}{x}-R^{2} x\right) \ln \left[1-R^{2} x(1-x)\right] .
$$

For $R \ll 1$ the function $J$ has the form

$$
J(R)=-\frac{R^{2}}{2}\left(1-\frac{R^{2}}{12}-\frac{R^{4}}{180}\right)+\mathcal{O}\left(R^{8}\right) .
$$

After squaring the amplitude (C.1) and summing over final state polarizations, we find the following decay width:

$$
\Gamma\left(\Theta^{+} \rightarrow W^{+} g\right)=\frac{\alpha_{s} \alpha \mu_{\Theta}^{2}}{\pi^{3} \sin ^{2} \theta_{W} M_{\Theta}} f(R)
$$




\section{CHAPTER 4. OCTOTRIPLETS}

where

$$
f(R)=\frac{1}{2} \mathcal{C}^{2}\left(1-R^{2}\right)
$$

with $\mathcal{C}$ depending on $R$ as shown in Eq. (C.4). This function, which appears in all 1loop decays discussed in this paper, is accidentally suppressed by cancellations between terms involving various powers of $\pi$. To see this, consider the expansion around $R \equiv$ $M_{W} / M_{\Theta} \rightarrow 0:$

$$
f(R)=f(0)+f_{1} R^{2}+f_{2} R^{4}+\mathcal{O}\left(R^{6}\right)
$$

Each of the above coefficients happens to be much smaller than order one:

$$
\begin{aligned}
f(0) & =\frac{9}{8}\left(\frac{\pi^{2}}{9}-1\right)^{2} \simeq 1.05 \times 10^{-2} \\
f_{1} & =f(0)+\frac{9}{4}\left(\frac{\pi^{2}}{9}-1\right)\left(\frac{\pi}{\sqrt{3}}-2\right) \\
& \simeq-3.00 \times 10^{-2}, \\
f_{2} & =\frac{9}{8}\left(\frac{\pi^{2}}{9}+\frac{\pi}{\sqrt{3}}-3\right)^{2}+\frac{3}{16}\left(\frac{\pi^{2}}{9}-1\right) \\
& \simeq 2.71 \times 10^{-2} .
\end{aligned}
$$

The above value of $f(0)$ agrees with that extracted from the width of an octo-doublet [127] or octo-singlet [117] decaying into $g g$.

Eqs. (C.7), (C.9) and (C.10) show that the 2-body decays of the octo-triplet into gauge bosons are suppressed by two orders of magnitude compared to estimates based on dimensional analysis. 


\section{Chapter 5}

\section{Very Light Axigluons}

\subsection{Introduction}

The CDF and D0 collaborations have recently reported measurements of the forwardbackward asymmetry $\left(A_{F B}\right)$ in $t \bar{t}$ production with intriguing deviations from the standard model prediction. CDF's result [15] in the lepton plus jets channel reports an inclusive parton level asymmetry

$$
A_{F B}(C D F)_{\ell j}=(15.8 \pm 7.4) \%
$$

If their measurement in the dilepton channel [16] is combined with this result, the asymmetry becomes

$$
A_{F B}(C D F)_{\ell \ell+\ell j}=(20.9 \pm 6.6) \%
$$




\section{CHAPTER 5. VERY LIGHT AXIGLUONS}

and exceeds the standard model prediction $\simeq 5 \%$ [17]- [19] by more than 2 standard deviations.

D0 performs a similar search [20] in the lepton plus jets channel and reports an inclusive parton-level asymmetry

$$
A_{F B}(D 0)_{\ell j}=(19.6 \pm 6.5) \%
$$

which is also more than $2 \sigma$ above the SM result. Taken together, these consistent deviations may be evidence for new physics in top quark production.

While all the inclusive measurements are consistent with each other, CDF's lepton plus jets search sees sharp mass dependence [15] in the binned result

$$
\begin{aligned}
& A_{F B}\left(M_{t \bar{t}}<450 \mathrm{GeV}\right)=(-11.6 \pm 14.6) \%, \\
& A_{F B}\left(M_{t \bar{t}}>450 \mathrm{GeV}\right)=(47.5 \pm 11.4) \%,
\end{aligned}
$$

where the high mass bin is $3.4 \sigma$ above the SM prediction. Neither D0 nor the complementary CDF dilepton search see the same effect; both find consistently positive $>2 \sigma$ deviations from the SM over the full $M_{t \bar{t}}$ range.

It has been observed that massive gluons with axial couplings can induce a large forward-backward asymmetry in $t \bar{t}$ production by interfering with standard model processes [154]- [166]. Motivated primarily by the mass dependent CDF result, these models predict asymmetries that rise uniformly with invariant mass and feature a sign flip near $M_{t \bar{t}} \approx 450 \mathrm{GeV}$. Large (TeV scale) masses are typically required to satisfy dijet-resonance search bounds and suppress contributions to the $t \bar{t}$ invariant mass distribution. To produce 


\section{CHAPTER 5. VERY LIGHT AXIGLUONS}

an asymmetry with the observed sign, most models also require flavor violation and are severely constrained [167] by limits on flavor changing neutral currents. For a comparison of heavy axigluons and other models that address the top asymmetry, see [168].

Relatively lighter axigluons (400 - $450 \mathrm{GeV}$ ) [169] can produce a large top asymmetry without flavor violation, but this mass scale is in tension with dijet resonance bounds and the differential $M_{t \bar{t}}$ distribution. Extra field content is generally required to broaden decay widths and avoid resonant enhancements to top quark observables.

In this paper we propose a very light $(50-90 \mathrm{GeV})$, weakly coupled axigluon to explain the top asymmetry. The model inherits many of the features heavier axigluons enjoy, but counterintuitively avoids their experimental constraints by being light: dijet resonance searches suffer from large QCD backgrounds at low invariant masses, particles below the $2 m_{t}$ threshold do not produce bumps in the $t \bar{t}$ invariant mass distribution, and nonresonant production suppresses new physics contributions to the $t \bar{t}$ cross section, which start at fourth order in the axigluon coupling. We find that the strongest upper bounds in this mass range come from Tevatron searches for light Higgs bosons produced in association with an additional $b$-jet. The strongest lower bounds come from UA2 dijet searches and LEP measurements of the hadronic $Z$ width.

In Section 5.2 we describe our model; in Section 5.3 we discuss the details of our numerical simulation; in Section 5.4 we address the experimental constraints; in Section 5.5 we compute the $t \bar{t}$ forward-backward asymmetry and compare theoretical predictions with production-level data; in Section 5.6 we make some concluding remarks. 


\section{CHAPTER 5. VERY LIGHT AXIGLUONS}

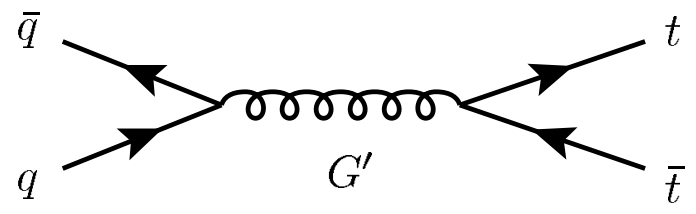

Figure 5.1: Axigluon contribution to $t \bar{t}$ pair production. Interference with the standard model gluon exchange diagram generates $\mathcal{A}_{\text {int }}^{G^{\prime}}$.

\subsection{Model Description}

We give the axigluon $\left(G^{\prime}\right)$ flavor universal couplings to SM quarks

$$
\mathcal{L} \supset g^{\prime} G_{\mu}^{a} \bar{Q} T^{a} \gamma^{\mu} \gamma^{5} Q
$$

where $g^{\prime} \equiv \lambda g_{s}$ is the axigluon coupling constant, which we express in units of the strong coupling. This operator can arise from an extended $S U(3)_{1} \times S U(3)_{2}$ color group that breaks down to the diagonal $S U(3)_{c}$ of QCD and gives rise to massive spin-1 color octets [170]- [173]. For an axigluon of mass $m_{G^{\prime}}$ our effective model requires a UV completion at the scale $4 \pi m_{G^{\prime}} / g^{\prime}=1.7 \mathrm{TeV}$ and $850 \mathrm{GeV}$ for $\lambda=0.3$ and 0.6 respectively. In this paper, we will focus only on the low energy effective theory and leave UV model building for future work.

Without additional field content, all decays proceed through operator in Eq. (C.1), so axigluons can only decay to quark pairs and give rise to dijet and four jet events for single and pair production, respectively. Since we work in the regime where the axigluon is below the $t \bar{t}$ threshold, the total width is [174]

$$
\Gamma_{G^{\prime}}=\frac{n_{f}}{6} \alpha_{s} \lambda^{2} m_{G^{\prime}}
$$




\section{CHAPTER 5. VERY LIGHT AXIGLUONS}

where $n_{f}$ is the number of active fermion flavors. For $m_{G^{\prime}}=80 \mathrm{GeV}$ and $\lambda=0.4$, this width is $\Gamma_{G^{\prime}} \simeq 1.1 \mathrm{GeV}$.

The differential cross section for the process $q \bar{q} \rightarrow t \bar{t}$ in the CM frame is a sum of standard model, interference, and axigluon terms

$$
\frac{d \hat{\sigma}\left(G^{\prime}\right)}{d \cos \theta}=\mathcal{A}_{S M}+\mathcal{A}_{\text {int }}^{G^{\prime}}+\mathcal{A}_{\text {axi }}^{G^{\prime}}
$$

where [175]

$$
\begin{aligned}
& \mathcal{A}_{S M}=\frac{\pi \alpha_{s}^{2} \beta}{9 \hat{s}}\left(2-\beta^{2}+(\beta \cos \theta)^{2}\right), \\
& \mathcal{A}_{\text {int }}^{G^{\prime}}=\frac{4 \pi \alpha_{s}^{2} \lambda^{2}}{9} \frac{\left(\hat{s}-m_{G^{\prime}}^{2}\right) \beta^{2} \cos \theta}{\left(\hat{s}-m_{G^{\prime}}^{2}\right)^{2}+m_{G^{\prime}}^{2} \Gamma_{G^{\prime}}^{2}}, \\
& \mathcal{A}_{\text {axi }}^{G^{\prime}}=\frac{\pi \alpha_{s}^{2} \lambda^{4}}{9} \frac{\hat{s} \beta^{3}\left(1+\cos ^{2} \theta\right)}{\left(\hat{s}-m_{G^{\prime}}^{2}\right)^{2}+m_{G^{\prime}}^{2} \Gamma_{G^{\prime}}^{2}} .
\end{aligned}
$$

Here $\beta \equiv \sqrt{1-4 m_{t}^{2} / \hat{s}}$ is the top quark velocity and $\theta$ is the angle between the incoming quark and outgoing top in the CM frame. A forward-backward asymmetry can only arise from terms with odd powers of $\cos \theta$, so the effect is due entirely to interference. In the presence of both vector and axial-vector couplings, there is an additional small contribution to the asymmetry from the new-physics squared term.

Note that the asymmetry generating term $\mathcal{A}_{\text {int }}^{G^{\prime}}$ is proportional to $\left(\hat{s}-m_{G^{\prime}}^{2}\right)$. For heavier axigluons, this dependence gives rise to a negative asymmetry because the mass is typically larger than the partonic CM energy. To compensate, many models introduce opposite sign 


\section{CHAPTER 5. VERY LIGHT AXIGLUONS}

couplings to the first and third generations. In our case, $m_{G^{\prime}}<\hat{s}$ for on-shell $t \bar{t}$ production, so the asymmetry is always positive and flavor violation is unnecessary.

\subsection{Simulation and Acceptances}

In the lepton plus jets analysis, $\mathrm{CDF}$ unfolds raw data by deconvolving their detector simulation and jet algorithm to yield a partonic data set from events that survive cuts at the detector level. To compare our model predictions with this data, it is necessary to generate an event sample with partonic $t \bar{t}$ pairs in the final state. However, knowing the predicted cross section and experimental luminosity is not enough to properly normalize kinematic distributions from the partonic simulation; we must also know the detector level acceptances. We thus perform two simulations: one at the partonic level to make our plots and one at the detector level with CDF's cuts to compute the acceptances that normalize these distributions.

We simulate the partonic process $p \bar{p} \rightarrow t \bar{t}$ in MadGraph 5 [176] using a model file generated with FeynRules [177]. This file adds the operator in Eq. (C.1) to the full standard model Lagrangian so that the process in Figure 5.1 contributes to $t \bar{t}$ production and gives rise to interference with SM gluon-exchange.

For the acceptances, we also perform a more realistic simulation $(p \bar{p} \rightarrow t \bar{t} \rightarrow \ell \nu+4 j)$ using Pythia [178] for the parton shower and PGS [179] for detector effects. To compare with CDF's lepton plus jets search, we impose the following cuts: at least four jets with 


\section{CHAPTER 5. VERY LIGHT AXIGLUONS}

$E_{T}>20 \mathrm{GeV}$ and at least one $b$-tag; for non- $b$ jets $\left|\eta_{j}\right|<2$, for $b$-jets $\left|\eta_{b j}\right|<1$; large missing energy $\mathbb{E}_{T}>20 \mathrm{GeV}$; and exactly one electron or muon with $p_{T}^{\ell}>20 \mathrm{GeV}$ and $\left|\eta_{\ell}\right|<1$.

Note that there is some error introduced by this approximate method. A complete comparison with experimental data would not only run a full detector simulation (including PYTHIA and PGS), but also identify top quarks with a least-squares kinematic fit and unfold the detector-level output using the $\mathrm{CDF}$ algorithm that reconstructs partonic events from raw data. Nonetheless, our approach accurately reproduces CDF's standard model expectation for the $t \bar{t}$ invariant mass distribution 1 so the error introduced by a constant acceptance function is likely to be small in our case as well. We leave the full unfolding for future work.

\subsection{Experimental Constraints}

Models that explain the top asymmetry must agree with the $t \bar{t}$ invariant mass distribution and total cross section, both of which are in good agreement with standard model predictions. Any candidate model with an $s$-channel mediator must satisfy constraints from dijet resonance searches at hadron colliders. In our case, we must also contend with a variety of older measurements that set lower bounds on new colored particles.

\footnotetext{
${ }^{1}$ Although the forward-backward asymmetry arises only at loop level in the SM, its numerical value is tiny $(\sim 5 \%)$, so this tree level method also adequately reproduces the (nearly symmetric) SM predictions for the $\Delta y=y_{t}-y_{\bar{t}}$ rapidity distributions in [15].
} 


\section{CHAPTER 5. VERY LIGHT AXIGLUONS}

\subsubsection{Top Quark Measurements}

The $t \bar{t}$ cross section at the Tevatron has been measured to be $\sigma_{t \bar{t}}^{\text {exp. }}=7.50 \pm 0.48$ $\mathrm{pb}[180]$, which agrees with the standard model prediction in perturbative QCD2, $\sigma_{t \bar{t}}^{s m} \simeq$ $(6.32-7.99) \mathrm{pb}$ for $m_{t}=172 \mathrm{GeV}$ [181]. The leading order result, $\left(\sigma_{t \bar{t}}^{s m}\right)_{L O} \simeq 5.63 \mathrm{pb}$ computed with MadGraph, implies a SM $K$-factor between 1.12 and 1.42.

Including an axigluon with $m_{G^{\prime}}=80 \mathrm{GeV}$ and $\lambda=0.4$, gives a total $\mathrm{LO}$ cross section of $\left(\sigma_{t \bar{t}}^{a x i}\right)_{L O}=6.08 \mathrm{pb}$, which is only an $8 \%$ increase over the SM LO result. This minor enhancement is due entirely to $\mathcal{A}_{a x i}^{G^{\prime}}$ in Eq. (C.1), which is fourth order in the axigluon coupling; the interference term $\mathcal{A}_{\text {int }}^{G^{\prime}}$ does not contribute to the total cross section. Although computing higher order corrections is beyond the scope of this work, the color structure of the axigluon exchange diagrams is identical to that of the relevant SM processes, so we expect higher order corrections to be of similar magnitude, though a more precise calculation is necessary to take into account the additional interference. As long as the $K$ factor does not differ substantially from that of SM production, the total $t \bar{t}$ cross section stays in good agreement with experiment. For the remainder of this paper, we will assume the $K$ factor to be 1.2 , so our benchmark cross section becomes $7.3 \mathrm{pb}$.

For very light axigluons $\left(m_{G^{\prime}} \ll 2 m_{t}\right)$, top pair production is nonresonant, so the invariant mass distribution is also in good agreement with experiment. In Figure 5.2 we show the simulated $M_{t \bar{t}}$ distribution (blue) plotted alongside the CDF data points and standard model background (purple) taken from the lepton plus jets search [15].

\footnotetext{
${ }^{2}$ For complementary calculations see $[182,183]$.
} 


\section{CHAPTER 5. VERY LIGHT AXIGLUONS}

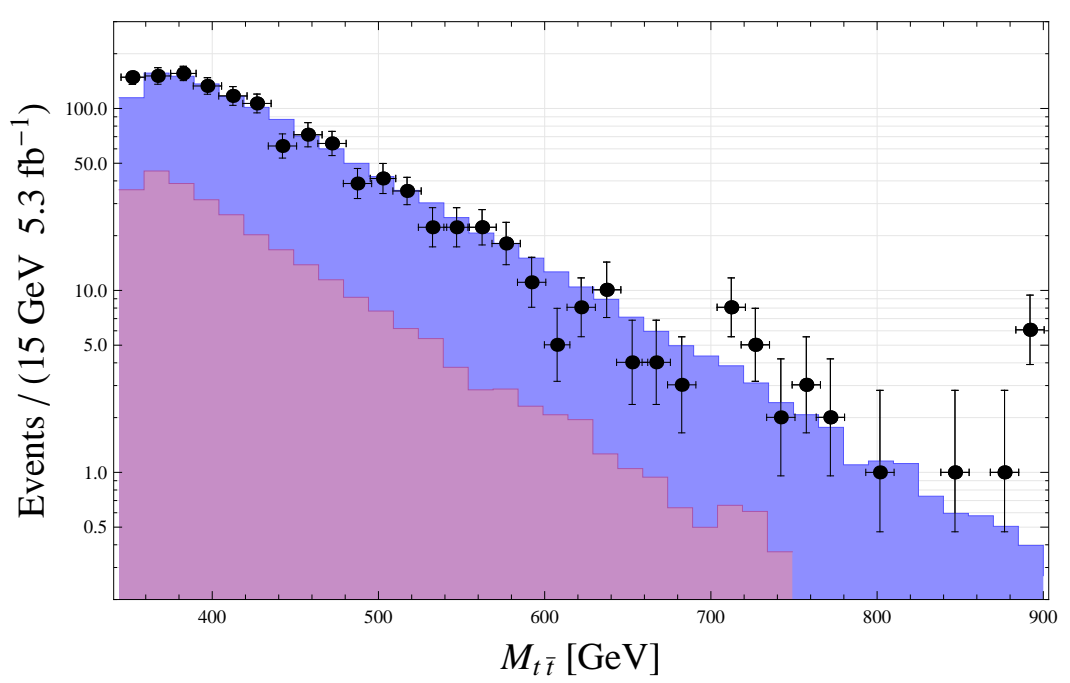

Figure 5.2: Tevatron invariant mass distribution for $t \bar{t}$ pairs (blue, color online) including both axigluon and background contributions. Data points and standard model background (purple) are taken from CDF's lepton plus jets search [15]. Here we use $\lambda=0.4$ and $m_{G^{\prime}}=80 \mathrm{GeV}$. After including a a $K$-factor of 1.2 , the top cross section is $\sigma_{t \bar{t}}=7.3 \mathrm{pb}$. Applying the CDF cuts (see Section 5.3) gives an acceptance of $2.6 \%$.

\subsubsection{Dijet Resonance Searches}

Quark coupled axigluons give rise to two and four jet events from single and pair production, respectively. Our mass range of interest $(50-90 \mathrm{GeV})$ is safe from Tevatron $[184,185]$ and LHC $[186,187]$ dijet resonance searches, which do not set bounds on masses below 180 and $200 \mathrm{GeV}$, respectively. A preliminary ATLAS analysis of multijet events [188] sets limits on color octet scalars with narrow widths, but does not constraint masses below $100 \mathrm{GeV}$. With lower search thresholds, this model may be testable at both the Tevatron and LHC, however, signal and background are expected to be large at both colliders [189].

The UA2 search for hadronic $W$ and $Z$ decays [190] measures the exclusive two-jet 


\section{CHAPTER 5. VERY LIGHT AXIGLUONS}

mass spectrum between 48 and $300 \mathrm{GeV}$, which constrains the light axigluon parameter

space. Using $4.7 p b^{-1}$ for $M_{j j}>66 \mathrm{GeV}$ (and $0.58 \mathrm{pb}^{-1}$ for $48 \mathrm{GeV}<M_{j j}<66 \mathrm{GeV}$ ), the combined $W$ and $Z$ resonances are extracted with a bi-gaussian fit above a smooth background function normalized to the data. The best fit bi-gaussian signal spans the $M_{j j}$ range between 70 and $100 \mathrm{GeV}$ and yields a cross section of $\sigma \cdot \mathcal{B} r(W, Z \rightarrow j j)_{\text {obs. }}=$ $9.6 \pm 2.3 \pm 1.1 \mathrm{nb}$, whose central value exceeds the $\mathrm{SM}$ prediction at $\mathrm{NLO}, \sigma \cdot \mathcal{B} r(W, Z \rightarrow$ $j j)_{S M}=5.8 \mathrm{nb}$, by almost a factor of two.

Although a three-gaussian fit and a QCD background prediction are necessary to properly constrain axigluons using this data, we can extract a rough bound by finding $\left(\lambda, m_{G^{\prime}}\right)$ values for which the combined SM and new-physics predictions exceed the observed number of events under the best fit gaussian by $2 \sigma$. In Figure 5.3 we plot the exclusion boundary (yellow dot-dashed line) determined using Madgraph, PythiA, and PGS to simulate our signal.

For dijet masses below $70 \mathrm{GeV}$, the UA2 analysis does not attempt to fit any signal, so a possible resonance would almost certainly have been missed given the very large background in this mass range. Even near $m_{W}$ and $m_{Z}$, the signal/background ratio is only a few percent and the gauge boson peak is not visible to the naked eye (see Figure 5 in [190]) prior to a rescaling that emphasizes the region around the known $W$ and $Z$ masses. Since the background model for this search is purely data-driven, the low-mass region does not impose a meaningful constraint without a dedicated bump hunt. 


\section{CHAPTER 5. VERY LIGHT AXIGLUONS}

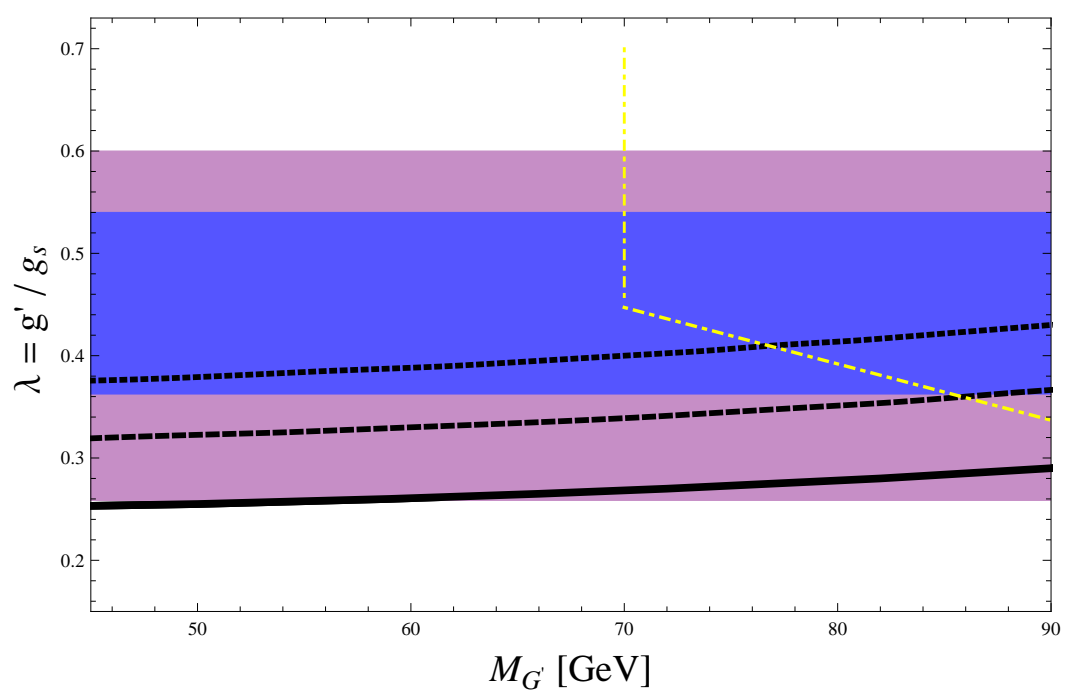

Figure 5.3: Allowed axigluon parameter space in the $\left(\lambda, m_{G^{\prime}}\right)$ plane plotted alongside bounds from dijet-resonance searches and $\Gamma(Z \rightarrow$ hadrons $)$ measurements assuming different extractions of $\alpha_{s}$. The blue and purple bands (color online) are regions favored by the combined CDF/D0 inclusive asymmetry measurements at $1 \sigma$ and $2 \sigma$, respectively. The dot-dashed yellow curve marks the approximate $2 \sigma$ bound above which model predictions exceed UA2 dijet limits from hadronic $W$ and $Z$ decays (see Section 5.4.2). The solid black curve marks the boundary above which corrections to the hadronic $Z$ width exceed the observed value by $2 \sigma$ assuming the standard model extraction of $\alpha_{s}\left(m_{Z}\right)=0.1184$. The dashed and dotted black curves give the same bound, but respectively assume $2.5 \%$ and $5 \%$ reductions to the $\mathrm{SM}$ value of $\alpha_{s}\left(m_{Z}\right)$. Reductions of this magnitude are typical of light axigluon contributions to the QCD beta function (for a discussion see Sections 5.4.5 and 5.4.6). The region above $m_{G^{\prime}}>90 \mathrm{GeV}$ is excluded by Tevatron $3 b$-searches. Since LEP event shapes rule out gluon-coupled adjoint fermions around $50 \mathrm{GeV}$, our model may encounter a stronger lower bound since axigluons also couple to quarks, but a proper analysis is necessary to set the correct limit.

\subsubsection{Light Higgs Searches}

Tevatron searches that look for light Higgs bosons produced in association with $b$-jets $(p \bar{p} \rightarrow h b \rightarrow b b b)$ are sensitive to axigluon decays into $b$-quarks. Since these searches require at least three $b$-tags to reduce the QCD multijet background, the bounds they impose on $\sigma(h b) \cdot \mathcal{B} r(h \rightarrow b b)$ also apply to the processes $p \bar{p} \rightarrow G^{\prime} b \rightarrow b b b$ and $p \bar{p} \rightarrow G^{\prime} b b \rightarrow$ 


\section{CHAPTER 5. VERY LIGHT AXIGLUONS}

$b b b b$, the latter of which can also arise from pair produced axigluons. However, the CDF [191] and D0 [192] results only apply to masses above $90 \mathrm{GeV}$; light axigluons fall below the sensitivity threshold. To be conservative, we will only consider masses below $90 \mathrm{GeV}$ where the $3 b$ constraints do not apply.

The authors in [193] use Tevatron Higgs searches in the associated production channel, $p \bar{p} \rightarrow W h \rightarrow(\ell \nu)(b \bar{b})$ to exclude axigluons with $\lambda=1$ between $75-125 \mathrm{GeV}$ assuming $\mathcal{B} r\left(G^{\prime} \rightarrow b \bar{b}\right)=1 / 5$. In our case with $\lambda=0.4$, the Tevatron $q \bar{q} \rightarrow W G^{\prime}$ cross section decreases by a factor of $\lambda^{2}$, which reduces the axigluon signal $\sigma \cdot \mathcal{B} r$ from $\approx 50 \mathrm{pb}$ down to $\approx 5 \mathrm{pb}$ for $m_{G^{\prime}}=50 \mathrm{GeV}$ also assuming $\mathcal{B} r\left(G^{\prime} \rightarrow b \bar{b}\right)=1 / 5$. This falls safely below the quoted bound of $\lesssim 20 \mathrm{pb}$, however, this number is based on analysis from an unpublished talk, so its status is not clear. Current Tevatron searches for the associated production of Higgs bosons are not sensitive to masses below $100 \mathrm{GeV}[194,195]$.

Naively it would appear that LEP searches in the Higgstrahlung channel [196]- [199] $e^{+} e^{-} \rightarrow Z h \rightarrow 4 j$ would be sensitive to light axigluons produced in $e^{+} e^{-} \rightarrow Z^{*} \rightarrow$ $q \bar{q} G^{\prime} \rightarrow 4 j$ events. However, the event selection algorithms in these analyses look for kinematics that fit the Higgstrahlung topology in which the invariant masses of jet pairs produce both $Z$ and Higgs resonances. In events with on-shell axigluons, all four jets arise from virtual $Z$ exchange, so this possibility is highly disfavored. Furthermore, this process occurs at order $\lambda^{2}$ and suffers additional phase-space suppression.

Similar considerations apply to LEP measurements of triple gauge boson couplings [200]- [203] which look for $e^{+} e^{-} \rightarrow W^{+} W^{-}, Z Z \rightarrow 4 j$ events. These analyses se- 


\section{CHAPTER 5. VERY LIGHT AXIGLUONS}

lect events using neural network algorithms designed to identify diboson production; light axigluons arising from $Z$ exchange have very different kinematics and fail this selection, which requires some combination of jet pairs to reconstruct at least one gauge boson mass. At the higher end of our mass range $\left(m_{G^{\prime}}>80 \mathrm{GeV}\right)$ it may be possible for an axigluon to fake a hadronically decaying SM gauge boson, but the other two jets would not reconstruct a resonance. The coupling and phase-space suppression also diminish the rate at these searches, so axigluon production is negligible compared to tree-level diboson and QCD background processes.

\subsubsection{Event Shapes}

Constraints on light colored-particles have been extracted from the analysis of event shapes at LEP. Comparing multijet data with calculations in soft colinear effective theory (SCET) rules out color adjoint fermions below $51 \mathrm{GeV}$ at $95 \%$ confidence [204]. However, this approach assumes that the new field couples only to gluons, with no tree-level quark interactions. To set a proper lower bound, it is necessary to repeat this analysis with more general assumptions, however, it is unlikely that this would yield a more lenient limit so we will not consider masses below $\approx 50 \mathrm{GeV}$.

LEP studies of four-jet events from $Z$ decays [205]- [208] can be sensitive to light, colored particles that couple to quarks. Various angular distributions are used to successfully distinguish $S U(3)_{c}$ QCD from alternative abelian theories of the strong force, so the

presence of light axigluons could potentially spoil this success. However, using Madgraph 


\section{CHAPTER 5. VERY LIGHT AXIGLUONS}

to generate four-jet $Z$ decays at the parton level, we find that the presence of an axigluon $(\lambda=0.4)$ in our mass range does not qualitatively distort these angular distributions relative to the QCD prediction. This is unsurprising since $\mathcal{O}(10 \%)$ of SM hadronic $Z$ decays produce four-jets - the exact number depends on $y_{c u t}$ and other jet algorithm details [209] whereas in our model only $\mathcal{O}(0.1 \%)$ of hadronic decays proceed through $Z \rightarrow q \bar{q} G^{\prime} \rightarrow 4 j$ prior to imposing cuts (see Section 5.4.6). For higher energies probed by LEP II $(\sqrt{s} \approx$ $200 \mathrm{GeV}$ ), the total $e^{+} e^{-} \rightarrow Z^{*} \rightarrow q \bar{q} G^{\prime} \rightarrow 4 j$ rate is similarly negligible compared to SM four jet production; this conclusion is robust for values of $y_{c u t}$ spanning several orders of magnitude.

\subsubsection{Running of $\alpha_{s}$}

Since axigluons couple to the strong sector, they give rise to loop diagrams that modify the QCD beta function above the scale $m_{G^{\prime}}$. The standard model running between energy scales $Q$ and $\mu$ is given by

$$
\alpha_{s}\left(Q^{2}\right)=\frac{\alpha_{s}\left(\mu^{2}\right)}{1+b \alpha_{s}\left(\mu^{2}\right) \log \left(\frac{Q^{2}}{\mu^{2}}\right)}
$$

where, to leading order, $b=\left(33-2 n_{f}\right) / 12 \pi$ and $n_{f}$ is the number of active flavors. Since axigluons have the same quantum numbers and self couplings as gluons, their principal effect on the running is to double the gluon contribution to the beta function above $m_{G^{\prime}}$ : $b \rightarrow\left(2 \times 33-2 n_{f}\right) / 12 \pi$. This accelerates asymptotic freedom and yields smaller values of $\alpha_{s}$ near the weak scale. 


\section{CHAPTER 5. VERY LIGHT AXIGLUONS}

While this adjustment naïvely jeopardizes the agreement between theory and experiment for the running, the experimental extraction of $\alpha_{s}$ depends entirely on the assumed validity of standard model QCD with no additional field content [210]. At each energy scale, an $\alpha_{s}$-dependent observable is equated to the SM prediction and the resulting data point is extracted implicitly. If light new states were present in the strong sector, this data would completely ignore their contributions, so the current agreement between theory and experiment does not constrain our model.

To roughly estimate the axigluon correction to $\alpha_{s}\left(m_{Z}\right)$, we use a well-measured value of $\alpha_{s}$ below $m_{G^{\prime}}$ as an IR boundary condition and evolve it with the new beta function. This method is crude because even low-energy observables used to extract $\alpha_{s}$ depend somewhat on virtual axigluon processes, which are ignored in the extraction of reported measurements. Nonetheless, using the boundary condition $\alpha_{s}(14.9 \mathrm{GeV})=0.160$, [210] the weakscale value becomes $\alpha_{s}\left(m_{Z}\right)=0.105,0.110$, and 0.115 for $m_{G^{\prime}}=50,65$ and $80 \mathrm{GeV}$, respectively. Different IR boundary conditions give similar downward corrections of order a few percent relative to the SM extraction $\alpha_{s}\left(m_{Z}\right)=0.1184$. Note that this result is independent of $\lambda$ since axigluons couple to gluons with QCD strength.

This model also predicts a kink in the running of $\alpha_{s}$ near $m_{G^{\prime}}$. Our mass range of interest $(50-90 \mathrm{GeV})$, however, overlaps with a region where data points are sparsely distributed with relatively large error bars (see Figure 6 in [210]) compared to the data set as a whole. Kinks in the slope of $\alpha_{s}$ would, therefore, be unlikely to stand out in the data. Nonetheless, a model-dependent extraction of $\alpha_{s}$ is necessary to evaluate the possibility of 


\section{CHAPTER 5. VERY LIGHT AXIGLUONS}

kinks or overall data shifts due to new physics contributions.

\subsubsection{Hadronic $Z$ Width}

The strongest lower bound on $m_{G^{\prime}}$ comes from virtual and three-body corrections to the hadronic $Z$ width. Axigluons that couple to quarks with QCD strength $(\lambda=1)$ enhance this width by a factor of

$$
1+\frac{\alpha_{s}}{\pi} f\left(m_{Z} / m_{G^{\prime}}\right)+\mathcal{O}\left(\alpha_{s}^{2}\right),
$$

where $f$ is a function derived in $[211,212]$. The LEP measurement of $\Gamma(Z \rightarrow$ hadrons $)$ and the extracted value of $\alpha_{s}\left(m_{Z}\right)$ constrain the size of $f\left(m_{Z} / m_{G^{\prime}}\right)$ and severely restrict axigluon masses: $m_{G^{\prime}}>570(365) \mathrm{GeV}$ for $\lambda=1$ at the $65 \%(95 \%)$ confidence level [193].

However, $f$ is highly nonlinear, so the mass constraint is extremely sensitive to the axigluon coupling. In our scenario, the constraint on $f$ applies to the combination $\lambda^{2} f$, which dramatically weakens the lower bound on $m_{G^{\prime}}$. Furthermore, following the discussion in Section 5.4.5, light axigluon $\left(m_{G^{\prime}}<m_{Z}\right)$ contributions to the QCD beta function generically decrease the value of $\alpha_{s}\left(m_{Z}\right)$ at the percent level. Since this is used to compute QCD corrections to the SM prediction for $\Gamma(Z \rightarrow$ hadrons $)$ [213], a smaller value opens up more allowed parameter space for new physics; the positive axigluon contribution to the width compensates for a slightly smaller SM result which is reduced by the new value of $\alpha_{s}$. 


\section{CHAPTER 5. VERY LIGHT AXIGLUONS}

In Figure 5.3 we plot $2 \sigma$ exclusion bounds from the hadronic $Z$ width on the $\left(\lambda, m_{G^{\prime}}\right)$ plane alongside the regions favored by combined CDF and D0 $A_{F B}$ measurements (discussed in Section 5.5). The solid black curve uses the standard model extraction $\alpha_{s}\left(m_{Z}\right)=$ $0.1184 \pm 0.0007$ [210] and the measured $\Gamma(Z \rightarrow$ hadrons $)=1.744 \pm 0.002 \mathrm{GeV}$ [214] to identify parameters for which the theoretical prediction exceeds the measured central value by $2 \sigma$. Also plotted are the $2 \sigma$ bounds assuming $2.5 \%$ (black dashed) and $5 \%$ (black dotted) reductions in $\alpha_{s}\left(m_{Z}\right)$ due to the modified running that includes axigluon contributions. These curves show how sensitive the bound is to modifications in $\lambda$ and $\alpha_{s}\left(m_{Z}\right)$. Since we generically expect light axigluons to reduce the value of $\alpha_{s}\left(m_{Z}\right)$ by a few percent relative to the SM extraction, the dashed and dotted curves are more faithful to the underlying physics. Given the sensitivity of the bound, a proper extraction of $\alpha_{s}$ involving axigluon processes is necessary to accurately constrain the parameter space; the limits in Figure 5.3 serve merely to illustrate the impact on the allowed region.

\subsubsection{Bounds from $\sigma\left(e^{+} e^{-} \rightarrow\right.$ hadrons $)$}

The authors in [211] calculate 3 axigluon corrections to the ratio

$$
R(s) \equiv \frac{\sigma\left(e^{+} e^{-} \rightarrow \text { hadrons }\right)}{e^{4} / 12 \pi s}
$$

at the scale $\sqrt{s}=34 \mathrm{GeV}$ and thereby exclude masses below $50 \mathrm{GeV}$ at $95 \%$ confidence assuming $\lambda=1$. As with the hadronic $Z$ width, the corrections for this process are propor-

\footnotetext{
${ }^{3}$ Note that [211] corrects some minor, yet consequential errors from an earlier paper [212] that placed a far stronger lower-bound on the mass.
} 


\section{CHAPTER 5. VERY LIGHT AXIGLUONS}

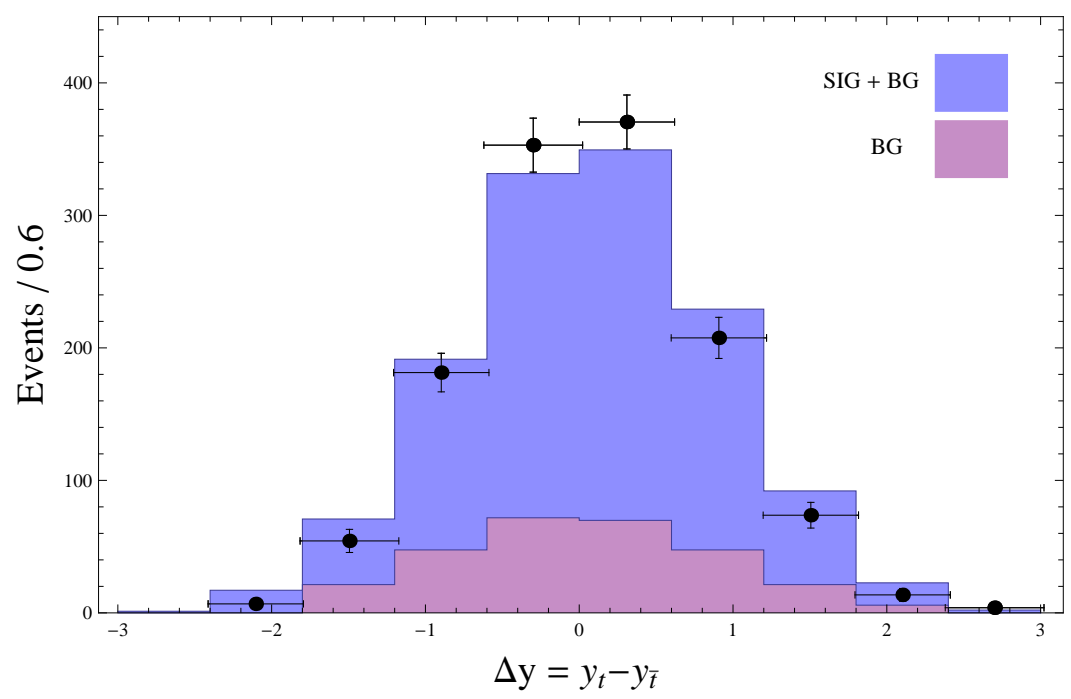

Figure 5.4: Inclusive top anti-top rapidity difference distribution plotted against unfolded CDF data. Here we use the same model parameters as in Fig. 5.2. The blue histograms include both signal and standard model background. Both data and background (purple) are taken from [15]. This plot omits the small, loop level asymmetry generated by SM processes.

tional to the factor in Eq. (C.2) with the replacement $\alpha_{s} \rightarrow \lambda^{2} \alpha_{s}$, so the discussion in Section 5.4.6 applies to this bound as well. Since $\Gamma(Z \rightarrow$ hadrons $)$ is extracted from $R$ data at the $Z$ pole, the allowed parameter space in Figure 5.3 is automatically consistent with bounds from $R$ near $\sqrt{s}=m_{Z}$. For smaller energies in our range of interest, $\sqrt{s} \in 50-90$ $\mathrm{GeV}$, the uncertainties on the $R$ data are larger than those at the $Z$ pole [214], so the bound is weaker. 


\subsection{Forward Backward Asymmetry}

The forward-backward asymmetry can be written

$$
A_{F B} \equiv \frac{N(\Delta y>0)-N(\Delta y<0)}{N(\Delta y>0)+N(\Delta y<0)},
$$

where $\Delta y \equiv y_{t}-y_{\bar{t}}$ is the rapidity difference between the top and anti-top quarks.

In Figure 5.3 we show the favored parameter space in the $\left(\lambda, m_{G^{\prime}}\right)$ plane. The blue (purple) band represents the region of $1 \sigma(2 \sigma)$ agreement with the combined CDF, Eq. $(\overline{C .2}$, and D0, Eq. (C.3) inclusive measurements. For typical points in these regions, the model predicts a positive asymmetry of order $20 \%$.

In Figure 5.4 we show the inclusive $t \bar{t}$ rapidity-difference distribution plotted against the CDF data. The signal simulation is identical to that used to generate Figure 5.2 with $m_{G^{\prime}}=80 \mathrm{GeV}$ and $\lambda=0.4$. After applying the cuts described in Section 5.3, the acceptance is is $2.6 \%$. This plot only depicts the effects of tree-level processes; the histograms do not include the small asymmetry induced by standard model processes. However, the numerical results in Fig. 5.3 include the full asymmetry with both SM and new physics contributions.

Although our simulation gives an acceptable fit to the rapidity data, some of the bins are more than $1 \sigma$ away from data points. We, however, do not expect perfect agreement at this level of analysis. The distribution in Figure 5.4 is a rough approximation of the full theory prediction which requires both a full CDF detector simulation and the subsequent unfolding for a proper comparison with data. 


\section{CHAPTER 5. VERY LIGHT AXIGLUONS}

In Figure 5.5 we show the theory prediction for the mass dependent asymmetry $A_{F B}\left(M_{t \bar{t}}\right)$ plotted alongside the unfolded CDF data. Like other light $s$ channel mediators, light axigluons predict a positive asymmetry throughout the whole range of invariant masses. While the agreement at low invariant mass is not ideal, neither D0 nor the CDF dilepton measurement observe strong mass dependence, so the significance of the massdependent data is not clear.

Note that in Figures 5.2, 5.4 and 5.5 we only compare the model to CDF results because their published distributions feature production-level data, which allow for a direct comparison with parton level simulations. Comparison with D0's distributions requires a detailed understanding of their detector simulation, which is beyond the scope of this work. Our conclusions have emphasized inclusive results from both collaborations since these are in better agreement with each other than the more controversial mass-dependent data.

\subsection{Conclusions}

We have shown that a light axigluon with flavor universal couplings can generate a large, positive $t \bar{t}$ asymmetry and naturally agrees with measurements of $d \sigma / d M_{t \bar{t}}$. The model has viable parameter space consistent with light Higgs bounds, dijet resonance searches and measurements of the hadronic $Z$ width.

For masses between $50-90 \mathrm{GeV}$ and quark couplings in the range $0.3 g_{s}-0.6 g_{s}$, the theoretical prediction for the parton-level top asymmetry is in good agreement with 


\section{CHAPTER 5. VERY LIGHT AXIGLUONS}

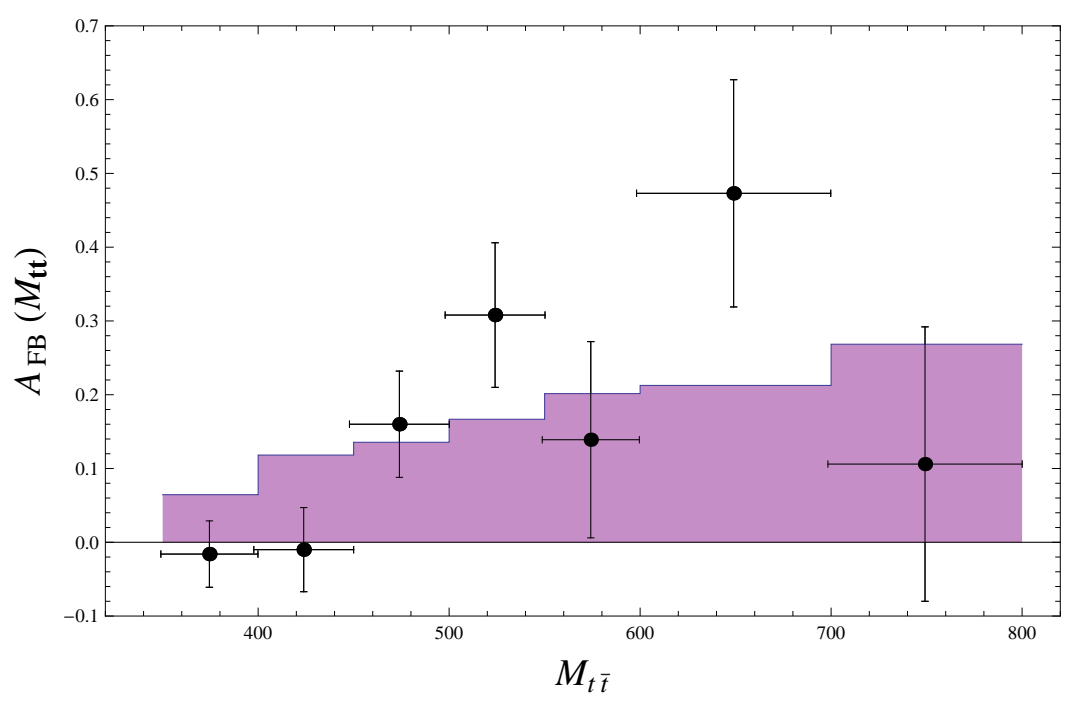

Figure 5.5: Theory prediction for the mass dependent $t \bar{t}$ asymmetry (purple histograms) plotted against the binned, unfolded CDF data in the lepton plus jets channel [15]. Here we use the same model parameters as in Fig. 5.2. For comparison with CDF, the bin sizes are $50 \mathrm{GeV}$ for $M_{t \bar{t}}<600 \mathrm{GeV}$ and $100 \mathrm{GeV}$ for larger invariant masses. Since the interference term in the differential cross section, Eq. (C.5), is proportional to $\left(\hat{s}-m_{G^{\prime}}^{2}\right)$, the asymmetry is always positive for on-shell $t \bar{t}$ production. This is a generic feature of light axigluon models.

inclusive results from both CDF and D0. The asymmetry is proportional to $\left(\hat{s}-m_{G^{\prime}}^{2}\right)$, so the sign of $A_{F B}$ is always positive for on shell top pair production with $\sqrt{s}>2 m_{t} \gg m_{G^{\prime}}$.

In the presence of a light axigluon, both the predicted and observed values of $\alpha_{s}$ are modified at the percent level. A reanalysis of $\alpha_{s}(\sqrt{s})$ measurements could reveal small downward shifts in the data since the modified beta function accelerates the running of $\alpha_{s}$ in the presence of an axigluon. The downward shift in $\alpha_{s}$ also decreases the SM predictions for $\Gamma(Z \rightarrow$ hadrons $)$ and $\sigma\left(e^{+} e^{-} \rightarrow\right.$ hadrons $)$, which expands the parameter space for $\left(\lambda, m_{G^{\prime}}\right)$ values that explain the top asymmetry.

Although the QCD background at low masses is formidable, it may be possible to revisit UA2 dijet data and perform a dedicated bump hunt in the low mass region with updated 


\section{CHAPTER 5. VERY LIGHT AXIGLUONS}

background calculations. It should also be possible to include light axigluons in a SCET reanalysis of event shapes in LEP data, which would likely set the strongest lower bound on this model.

If very light axigluons explain the top forward-backward asymmetry, the Tevatron and LHC experiments should, in principle, be able to observe resonances in two and four jet events from single and pair production. Since the effective model presented in this paper demands a UV completion at energy scales near the LHC's designed sensitivity, we predict new physics around the TeV scale, but the specific signals are model dependent at this level of description and would be interesting to pursue as future work. 


\section{Bibliography}

[1] ALEPH and DELPHI and L3 and OPAL and SLD and LEP Electroweak Working Group and SLD Electroweak Group and SLD Heavy Flavour Group Collaborations, Phys. Rept. 427, 257 (2006) [hep-ex/0509008].

[2] E. Accomando et al. [ECFA/DESY LC Physics Working Group Collaboration], Phys. Rept. 299, 1 (1998) [hep-ph/9705442].

[3] D. Larson, J. Dunkley, G. Hinshaw, E. Komatsu, M. R. Nolta, C. L. Bennett, B. Gold and M. Halpern et al., Astrophys. J. Suppl. 192, 16 (2011) [arXiv:1001.4635 [astroph.CO]].

[4] K. Abazajian et al. [SDSS Collaboration], Astrophys. J. 625, 613 (2005) [astro$\mathrm{ph} / 0408003]$.

[5] F. Zwicky, Astrophys. J. 86, 217 (1937).

[6] V. C. Rubin, N. Thonnard and W. K. Ford, Jr., Astrophys. J. 238, 471 (1980).

[7] E. Battaner and E. Florido, Fund. Cosmic Phys. 21, 1 (2000) [astro-ph/0010475]. 


\section{BIBLIOGRAPHY}

[8] D. Huterer, Gen. Rel. Grav. 42, 2177 (2010) [arXiv:1001.1758 [astro-ph.CO]].

[9] R. Bernabei et al. [DAMA Collaboration], Eur. Phys. J. C 56, 333 (2008) [arXiv:0804.2741 [astro-ph]].

[10] F. Petriello and K. M. Zurek, JHEP 0809, 047 (2008) [arXiv:0806.3989 [hep-ph]].

[11] D. Tucker-Smith and N. Weiner, Phys. Rev. D 64, 043502 (2001) [arXiv:hep$\mathrm{ph} / 0101138]$.

[12] T. Aaltonen et al. [CDF Collaboration], "Invariant Mass Distribution of Jet Pairs Produced in Association with a $W$ boson in $p \bar{p}$ Collisions at $\sqrt{s}=1.96 \mathrm{TeV}$," arXiv:1104.0699.

[13] V. M. Abazov et al. [D0 Collaboration], "Study of the dijet invariant mass distribution in $p \bar{p} \rightarrow W(\rightarrow \ell \nu)+j j$ final states at $\sqrt{s}=1.96 \mathrm{TeV}$,' arXiv:1106.1921 [hep-ex].

[14] For a review, see C. T. Hill and E. H. Simmons, "Strong dynamics and electroweak symmetry breaking,” Phys. Rept. 381, 235 (2003) [Erratum-ibid. 390, 553 (2004)] [arXiv:hep-ph/0203079].

[15] T. Aaltonen et al. [CDF Collaboration], Phys. Rev. D83, 112003 (2011), [arXiv:1101.0034].

[16] CDF Public Note, [10436]. 


\section{BIBLIOGRAPHY}

[17] J. H. Kuhn and G. Rodrigo, Phys. Rev. D 59, 054017 (1999), [arXiv: 9807420].

[18] V. Ahrens, A. Ferroglia, M. Neubert, B. D. Pecjak and L. L. Yang, arXiv:1106.6051].

[19] M. T. Bowen, S. D. Ellis, D. Rainwater, Phys. Rev. D73, 014008 (2006), [arXiv:0509267].

[20] D0 Collaboration, [arXiv:1107.4995].

[21] E. Komatsu et al. [WMAP Collaboration], Astrophys. J. Suppl. 180, 330 (2009) [arXiv:0803.0547 [astro-ph]].

[22] J. F. Navarro, C. S. Frenk and S. D. M. White, Astrophys. J. 462, 563 (1996) [arXiv:astro-ph/9508025].

[23] G. Gilmore, M. I. Wilkinson, R. F. G. Wyse, J. T. Kleyna, A. Koch, N. W. Evans and E. K. Grebel, Astrophys. J. 663, 948 (2007) [arXiv:astro-ph/0703308].

[24] Z. Ahmed et al. [CDMS Collaboration], Phys. Rev. Lett. 102, 011301 (2009) [arXiv:0802.3530 [astro-ph]].

[25] J. Angle et al. [XENON Collaboration], Phys. Rev. Lett. 100, 021303 (2008) [arXiv:0706.0039 [astro-ph]].

[26] O. Adriani et al. [PAMELA Collaboration], Nature 458, 607 (2009) [arXiv:0810.4995 [astro-ph]]. 


\section{BIBLIOGRAPHY}

[27] J. Chang et al., Nature 456, 362 (2008).

[28] S. Torii et al. [PPB-BETS Collaboration], arXiv:0809.0760 [astro-ph].

[29] A. A. Abdo et al. [The Fermi LAT Collaboration], Phys. Rev. Lett. 102, 181101 (2009) [arXiv:0905.0025 [astro-ph.HE]].

[30] C. Amsler et al. [Particle Data Group], Phys. Lett. B 667, 1 (2008).

[31] D. E. Kaplan, M. A. Luty and K. M. Zurek, arXiv:0901.4117 [hep-ph].

[32] G. R. Farrar and G. Zaharijas, Phys. Rev. Lett. 96, 041302 (2006) [arXiv:hep$\mathrm{ph} / 0510079]$.

[33] J. L. Feng, M. Kaplinghat, H. Tu and H. B. Yu, arXiv:0905.3039 [hep-ph].

[34] L. Ackerman, M. R. Buckley, S. M. Carroll and M. Kamionkowski, Phys. Rev. D 79, 023519 (2009) [arXiv:0810.5126 [hep-ph]].

[35] N. Kaloper and A. Padilla, Levitating Dark Matter, JCAP 0910 (2009) 023, [arXiv:0904.2394].

[36] D.-C. Dai, K. Freese, and D. Stojkovic, Constraints on dark matter particles charged under a hidden gauge group from primordial black holes, JCAP 0906 (2009) 023, [arXiv:0904.3331].

[37] D. S. M. Alves, S. R. Behbahani, P. Schuster and J. G. Wacker, arXiv:0903.3945 [hep-ph]. 


\section{BIBLIOGRAPHY}

[38] Z. Berezhiani, arXiv:hep-ph/0508233.

[39] R. N. Mohapatra, S. Nussinov and V. L. Teplitz, Phys. Rev. D 66, 063002 (2002) [arXiv:hep-ph/0111381].

[40] A. A. Klypin, A. V. Kravtsov, O. Valenzuela and F. Prada, Astrophys. J. 522, 82 (1999) [arXiv:astro-ph/9901240].

[41] B. Moore, S. Ghigna, F. Governato, G. Lake, T. R. Quinn, J. Stadel and P. Tozzi, Astrophys. J. 524, L19 (1999).

[42] P. J. E. Peebles, Astrophys. J. 153, 1 (1968).

[43] S. Dodelson, Modern Cosmology (Academic Press, San Diego, 2003).

[44] C. P. Ma and E. Bertschinger, Astrophys. J. 455, 7 (1995) [arXiv:astro-ph/9506072].

[45] L. Spitzer 1978, Physical Processes in the Interstellar Medium, ( Wiley, New York)

[46] L. J. Spitzer and J. L. Greenstein Astrophys. J. 114, 407 (1952).

[47] L. D. Landau and E. M. Lifshitz, Quantum Mechanics: Non-Relativistic Theory (Pergamon Press Ltd., London - Paris, 1958.)

[48] T. Wu and T. Ohmura, Quantum Theory of Scattering(Prentice-Hall Inc., New Jersey, 1962.)

[49] B.H. Bransden and C.J. Joachain, Physics of Atoms and Molecules(Addison Wesley Longman Ltd., Essex, 1983.) 


\section{BIBLIOGRAPHY}

[50] D. R. Schultz, P. S. Krstic, T. G. Lee and L. C. Raymond Astrophys. J. 114, 407 (1952).

[51] P. S. Krstic, and D .R. Schultz J. Phys. B 323485 (1999)

[52] P. S. Krstic, J. H. Macek, S. Yu. Ovchinnikov, and D. R. Schultz Phys. Rev. A 70 $042711(2004)$

[53] M. Markevitch et al., Astrophys. J. 606, 819 (2004) [arXiv:astro-ph/0309303].

[54] S. W. Randall, M. Markevitch, D. Clowe, A. H. Gonzalez and M. Bradac, arXiv:0704.0261 [astro-ph].

[55] J. Miralda-Escude, arXiv:astro-ph/0002050.

[56] A. Loeb and M. Zaldarriaga, Phys. Rev. D 71, 103520 (2005) [arXiv:astro$\mathrm{ph} / 0504112]$.

[57] D. Hooper, M. Kaplinghat, L. E. Strigari and K. M. Zurek, Phys. Rev. D 76, 103515 (2007) [arXiv:0704.2558 [astro-ph]].

[58] J. J. Sakurai, Modern Quantum Mechanics (Addison-Wesley Publishing Company, 1995), Revised

[59] R. Shankar, Principles of Quantum Mechanics (Springer, 1994)

[60] R. H. Helm, Phys. Rev. 104, 1466 (1956). 


\section{BIBLIOGRAPHY}

[61] G. Jungman, M. Kamionkowski and K. Griest, Phys. Rept. 267, 195 (1996) [arXiv:hep-ph/9506380].

[62] J. D. Lewin and P. F. Smith, Astropart. Phys. 6, 87 (1996).

[63] C. Savage, K. Freese and P. Gondolo, Phys. Rev. D 74, 043531 (2006) [arXiv:astro$\mathrm{ph} / 0607121]$.

[64] M. Pospelov, arXiv:0811.1030 [hep-ph].

[65] S. Davidson, S. Hannestad and G. Raffelt, JHEP 0005, 003 (2000) [arXiv:hep$\mathrm{ph} / 0001179]$.

[66] S. Chang, G. D. Kribs, D. Tucker-Smith and N. Weiner, Phys. Rev. D 79, 043513 (2009) [arXiv:0807.2250 [hep-ph]].

[67] M. E. Machacek and M. T. Vaughn, Nucl. Phys. B 236, 221 (1984).

[68] B. D. Wandelt, R. Dave, G. R. Farrar, P. C. McGuire, D. N. Spergel and P. J. Steinhardt, arXiv:astro-ph/0006344.

[69] R. Dave, D. N. Spergel, P. J. Steinhardt and B. D. Wandelt, Astrophys. J. 547, 574 (2001) [arXiv:astro-ph/0006218].

[70] C. M. Hirata and N. Padmanabhan, Mon. Not. Roy. Astron. Soc. 372, 1175 (2006) [arXiv:astro-ph/0606437]. 


\section{BIBLIOGRAPHY}

[71] N. Yoshida, K. Omukai, L. Hernquist and T. Abel, Astrophys. J. 652, 6 (2006) [arXiv:astro-ph/0606106].

bibitemBertone:2004pz G. Bertone, D. Hooper, and J. Silk, Particle dark matter: Evidence, candidates and constraints, Phys.Rept. 405 (2005) 279-390, [hep-ph/0404175].

[72] G. Mangano, A. Melchiorri, O. Mena, G. Miele and A. Slosar, Present bounds on the relativistic energy density in the Universe from cosmological observables, JCAP 0703, 006 (2007) [arXiv:astro-ph/0612150].

[73] J. F. Navarro, C. S. Frenk, and S. D. M. White, The Structure of Cold Dark Matter Halos, Astrophysical Journal 462 (May, 1996) 563, [astro-ph/9508025].

[74] G. Gilmore, M. I. Wilkinson, R. F. G. Wyse, J. T. Kleyna, A. Koch, N. W. Evans and E. K. Grebel, The Observed properties of Dark Matter on small spatial scales, Astrophys. J. 663, 948 (2007) [arXiv:astro-ph/0703308].

[75] CoGeNT collaboration, C. Aalseth et. al., Results from a Search for Light-Mass Dark Matter with a P-type Point Contact Germanium Detector, arXiv:1002.4703.

[76] D. P. Finkbeiner and N. Weiner, Exciting Dark Matter and the INTEGRAL/SPI 511 keV signal, Phys.Rev. D76 (2007) 083519, astro-ph/0 702587. 


\section{BIBLIOGRAPHY}

[77] M. Pospelov, A. Ritz, and M. B. Voloshin, Secluded WIMP Dark Matter, Phys.Lett. B662 (2008) 53-61, arXiv:0711.4866.

[78] K. M. Zurek, Multi-Component Dark Matter, Phys.Rev. D79 (2009) 115002, [arXiv:0811.4429].

[79] D. B. Kaplan, A Single explanation for both the baryon and dark matter densities, Phys. Rev. Lett. 68, 741 (1992).

[80] S. Nussinov, Technocosmology: Could A Technibaryon Excess Provide A 'natural' Missing Mass Candidate?, Phys. Lett. B 165, 55 (1985).

[81] S. M. Barr, Baryogenesis, sphalerons and the cogeneration of dark matter, Phys. Rev. D 44, 3062 (1991).

[82] S. M. Barr, R. S. Chivukula, E. Farhi, Electroweak Fermion Number Violation And The Production Of Stable Particles In The Early Universe, Phys. Lett. B241, 387391 (1990).

[83] S. B. Gudnason, C. Kouvaris and F. Sannino, Towards working technicolor: Effective theories and dark matter, Phys. Rev. D 73, 115003 (2006) [arXiv:hep-ph/0603014].

[84] S. Dodelson, B. R. Greene and L. M. Widrow, Baryogenesis, dark matter and the width of the Z, Nucl. Phys. B 372, 467 (1992).

[85] V. A. Kuzmin, A Simultaneous solution to baryogenesis and dark matter problems, 


\section{BIBLIOGRAPHY}

Phys. Part. Nucl. 29, 257 (1998) [Fiz. Elem. Chast. Atom. Yadra 29, 637 (1998)] [Phys. Atom. Nucl. 61, 1107 (1998)] [arXiv:hep-ph/9701269].

[86] M. Fujii and T. Yanagida, A Solution to the coincidence puzzle of Omega(B) and Omega (DM), Phys. Lett. B 542, 80 (2002) [arXiv:hep-ph/0206066].

[87] R. Kitano and I. Low, Dark matter from baryon asymmetry, Phys. Rev. D 71, 023510 (2005) [arXiv:hep-ph/0411133].

[88] G. R. Farrar and G. Zaharijas, Dark matter and the baryon asymmetry, Phys. Rev. Lett. 96 (2006) 041302, [hep-ph/0510079].

[89] R. Kitano, H. Murayama and M. Ratz, Unified origin of baryons and dark matter, Phys. Lett. B 669, 145 (2008) [arXiv:0807.4313 [hep-ph]].

[90] D. E. Kaplan, M. A. Luty, and K. M. Zurek, Asymmetric Dark Matter, Phys.Rev. D79 (2009) 115016, [arXiv:0901.4117].

[91] G. D. Kribs, T. S. Roy, J. Terning and K. M. Zurek, Quirky Composite Dark Matter, Phys. Rev. D 81, 095001 (2010) [arXiv:0909.2034 [hep-ph]].

[92] A. Falkowski, J. T. Ruderman, and T. Volansky, Asymmetric Dark Matter from Leptogenesis, arXiv:1101.4936, * Temporary entry*.

[93] M. A. Luty, Baryogenesis via leptogenesis, Phys. Rev. D45, 455-465 (1992).

[94] M. R. Buckley, Asymmetric Dark Matter and Effective Operators, arXiv:1104.1429. 


\section{BIBLIOGRAPHY}

[95] CDMS-II Collaboration, Z. Ahmed et. al., Results from a Low-Energy Analysis of the CDMS II Germanium Data, Phys.Rev.Lett. (2010) [arXiv:1011.2482].

[96] XENON10 Collaboration, J. Angle et. al., Constraints on inelastic dark matter from XENON10, Phys. Rev. D80 (2009) 115005, [arXiv:0910.3698].

[97] J. Angle et al., A search for light dark matter in XENON10 data, [arXiv:1104.3088].

[98] R. Bernabei, P. Belli, F. Cappella, R. Cerulli, C. Dai, et. al., Particle Dark Matter in DAMA/LIBRA, arXiv:1007.0595, * Temporary entry *.

[99] J. I. Collar and D. N. McKinsey, Comments on 'First Dark Matter Results from the XENON100 Experiment', arXiv:1005.0838.

[100] T. X. Collaboration, Reply to the Comments on the XENON100 First Dark Matter Results, arXiv:1005.2615.

[101] J. I. Collar and D. N. McKinsey, Response to arXiv:1005.2615, arXiv:1005.3723.

[102] R. Essig, J. Kaplan, P. Schuster, and N. Toro, On the Origin of Light Dark Matter Species, Submitted to Physical Review D (2010) [arXiv:1004.0691].

[103] S. Chang, A. Pierce, and N. Weiner, Momentum Dependent Dark Matter Scattering, JCAP 1001 (2010) 006, [arXiv:0908.3192]. 


\section{BIBLIOGRAPHY}

[104] J. L. Feng, J. Kumar, D. Marfatia, and D. Sanford, Isospin-Violating Dark Matter, arXiv:1102.4331.

[105] S. Chang, J. Liu, A. Pierce, N. Weiner, and I. Yavin, CoGeNT Interpretations, JCAP 1008 (2010) 018, [arXiv:1004.0697].

[106] D. E. Kaplan, G. Z. Krnjaic, K. R. Rehermann, and C. M. Wells, Atomic Dark Matter, JCAP 1005 (2010) 021, arXiv:0909.0753].

[107] See, for example, the talks by W. Seidel http://wonder.lngs.infn.it/templates/wm_06_j15/download/Seidel_CRE at WONDER2010 and F. Probsthttp://phy-gzk .princeton.edu/DMworkshop/Fran:

[108] B. Holdom, Two U(1)'s and Epsilon Charge Shifts, Phys. Lett. B166 (1986) 196.

[109] M. Pospelov, Secluded U(1) below the weak scale, Phys. Rev. D80 (2009) 095002, [arXiv:0811.1030].

[110] J. D. Bjorken, R. Essig, P. Schuster, and N. Toro, New Fixed-Target Experiments to Search for Dark Gauge Forces, Phys. Rev. D80 (2009) 075018, [arXiv:0906.0580].

[111] J. Redondo and A. Ringwald, Light shining through walls, arXiv:1011.3741, * Temporary entry *.

[112] K. Ichikawa, T. Sekiguchi and T. Takahashi, Probing the Effective Number of Neu- 


\section{BIBLIOGRAPHY}

trino Species with Cosmic Microwave Background,' Phys. Rev. D 78, 083526 (2008) [arXiv:0803.0889].

[113] J. Einasto and U. Haud, Galactic models with massive corona. I - Method. II Galaxy, Astronomy and Astrophysics 223 (Oct. 1989) 89-106.

[114] R. Catena and P. Ullio, A novel determination of the local dark matter density, JCAP 1008 (2010) 004, [arXiv:0907.0018].

[115] D. Hooper, J. I. Collar, J. Hall and D. McKinsey, A Consistent Dark Matter Interpretation For CoGeNT and DAMA/LIBRA, Phys. Rev. D 82, 123509 (2010) [arXiv:1007.1005 [hep-ph]].

[116] R. S. Chivukula, M. Golden and E. H. Simmons, "Multi - jet physics at hadron colliders," Nucl. Phys. B 363, 83 (1991).

[117] B. A. Dobrescu, K. Kong and R. Mahbubani, "Massive color-octet bosons and pairs of resonances at hadron colliders," Phys. Lett. B 670, 119 (2008) [arXiv:0709.2378 [hep-ph]].

[118] Y. Bai, B. A. Dobrescu, "Heavy octets and Tevatron signals with three or four $b$ jets," [arXiv:1012.5814 [hep-ph]].

[119] CDF Collaboration, "Search for Higgs bosons produced in association with $b$ quarks", Note 10105, June 2010, 


\section{BIBLIOGRAPHY}

http://www-cdf.fnal.gov/physics/new/hdg//

Results_files/results/3b_susyhiggs_jun10

[120] R. Boughezal, F. Petriello, "Color-octet scalar effects on Higgs boson production in gluon fusion,” Phys. Rev. D81, 114033 (2010). [arXiv:1003.2046 [hep-ph]].

R. Boughezal, “Constraints on heavy colored scalars from Tevatron's Higgs exclusion limit," [arXiv:1101.3769 [hep-ph]].

[121] C. Kilic, T. Okui and R. Sundrum, "Colored Resonances at the Tevatron: Phenomenology and Discovery Potential in Multijets," JHEP 0807, 038 (2008) [arXiv:0802.2568 [hep-ph]]. "Vectorlike Confinement at the LHC," JHEP 1002, 018 (2010) [arXiv:0906.0577 [hep-ph]].

[122] Y. Bai and A. Martin, “Topological Pions," Phys. Lett. B 693, 292 (2010) [arXiv:1003.3006].

Y. Bai and R. J. Hill, “Weakly interacting stable hidden sector pions,” Phys. Rev. D 82, 111701 (2010) [arXiv:1005.0008 [hep-ph]].

[123] G. Burdman, B. A. Dobrescu and E. Ponton, "Resonances from two universal extra dimensions," Phys. Rev. D 74, 075008 (2006) [arXiv:hep-ph/0601186].

[124] M. V. Martynov and A. D. Smirnov, "Chiral color symmetry and possible $G^{\prime}$ boson effects at the Tevatron and LHC," Mod. Phys. Lett. A 24, 1897 (2009) [arXiv:0906.4525 [hep-ph]]. 


\section{BIBLIOGRAPHY}

[125] A. V. Manohar and M. B. Wise, "Flavor changing neutral currents, an extended scalar sector, and the Higgs production rate at the LHC," Phys. Rev. D 74, 035009 (2006) [arXiv:hep-ph/0606172].

[126] J. M. Arnold, M. Pospelov, M. Trott and M. B. Wise, "Scalar representations and minimal flavor violation,” JHEP 1009, 073 (2010). [arXiv:0911.2225 [hep-ph]].

[127] M. I. Gresham, M. B. Wise, "Color octet scalar production at the LHC," Phys. Rev. D76, 075003 (2007). [arXiv:0706.0909 [hep-ph]].

[128] M. Gerbush et al., "Color-octet scalars at the LHC," Phys. Rev. D77, 095003 (2008). [arXiv:0710.3133].

[129] CDF Collaboration, http://www-cdf.fnal.gov/ physics/ewk/2011/wjj/7_3.html, May 2011.

[130] F. Yu, “A $Z^{\prime}$ model for the CDF dijet anomaly," arXiv:1104.0243.

M. R. Buckley, D. Hooper, J. Kopp and E. Neil, “Light Z' bosons at the Tevatron,” arXiv:1103.6035.

K. Cheung, J. Song, "Tevatron Wjj Anomaly and the baryonic $Z^{\prime}$ solution," arXiv:1104.1375.

X. P. Wang et al, "New color-octet vector boson revisit," arXiv:1104.1917; "O(100 GeV) deci-weak $W^{\prime} / Z^{\prime}$ at Tevatron and LHC," arXiv:1104.1161.

L. A. Anchordoqui et al, "Stringy origin of Tevatron Wjj anomaly," arXiv:1104.2302. 


\section{BIBLIOGRAPHY}

[131] A. E. Nelson, T. Okui, T. S. Roy, "A unified, flavor symmetric explanation for the $t \bar{t}$ asymmetry and Wjj excess at CDF," arXiv:1104.2030.

C. Kilic and S. Thomas, "Signatures of resonant super-partner production with charged-current decays," arXiv:1104.1002.

E. J. Eichten, K. Lane and A. Martin, "Technicolor at the Tevatron," arXiv:1104.0976.

[132] R. Sato, S. Shirai, K. Yonekura, "A Possible Interpretation of CDF Dijet Mass Anomaly and its Realization in Supersymmetry," arXiv:1104.2014.

[133] S. Dodelson, B. R. Greene, L. M. Widrow, "Baryogenesis, dark matter and the width of the Z," Nucl. Phys. B372, 467-493 (1992).

M. Cirelli, N. Fornengo, A. Strumia, “Minimal dark matter,” Nucl. Phys. B753, 178194 (2006). [hep-ph/0512090].

[134] J. Alwall, M. Herquet, F. Maltoni, O. Mattelaer, T. Stelzer, "MadGraph 5 : Going Beyond," [arXiv:1106.0522 [hep-ph]].

[135] N. D. Christensen, C. Duhr, "FeynRules - Feynman rules made easy," Comput. Phys. Commun. 180, 1614-1641 (2009). [arXiv:0806.4194]; FeynRules, version 1.6beta, http://feynrules.irmp.ucl.ac.be/;

Model files for heavy octets are available at http://theory.fnal.gov/people/dobrescu/octet/. 


\section{BIBLIOGRAPHY}

[136] J. Pumplin et al., "New generation of parton distributions with uncertainties from global QCD analysis,”JHEP 0207, 012 (2002) [arXiv:hep-ph/0201195].

[137] Y. Kats, M. D. Schwartz, "Annihilation decays of bound states at the LHC," JHEP 1004, 016 (2010). [arXiv:0912.0526 [hep-ph]].

[138] C. Kim, T. Mehen, "Color Octet Scalar Bound States at the LHC," Phys. Rev. D79, 035011 (2009). [arXiv:0812.0307 [hep-ph]].

A. Idilbi, C. Kim, T. Mehen, "Pair Production of Color-Octet Scalars at the LHC," Phys. Rev. D82, 075017 (2010). [arXiv:1007.0865].

[139] E. Gamiz et al. [HPQCD Collaboration], "Neutral $B$ meson mixing in unquenched lattice QCD,’ Phys. Rev. D 80, 014503 (2009) [arXiv:0902.1815 [hep-lat]].

[140] A. J. Buras, S. Jager and J. Urban, "Master formulae for $\Delta F=2$ NLO-QCD factors in the standard model and beyond," Nucl. Phys. B 605, 600 (2001) [arXiv:hep$\mathrm{ph} / 0102316]$.

[141] D. Becirevic et al., " $B$-parameters of the complete set of matrix elements of $\Delta B=2$ operators from the lattice," JHEP 0204, 025 (2002) [arXiv:hep-lat/0110091].

[142] A. Lenz and U. Nierste, “Theoretical update of $B_{s}-\bar{B}_{s}$ mixing," JHEP 0706, 072 (2007) [arXiv:hep-ph/0612167].

[143] B. A. Dobrescu, P. J. Fox, A. Martin, "CP violation in $B_{s}$ mixing from heavy Higgs exchange,” Phys. Rev. Lett. 105, 041801 (2010). [arXiv:1005.4238 [hep-ph]]. 


\section{BIBLIOGRAPHY}

[144] V. M. Abazov et al. [D0 Collaboration], "Evidence for an anomalous like-sign dimuon charge asymmetry," Phys. Rev. D 82, 032001 (2010) [arXiv:1005.2757 [hepex]].

[145] CDF Collaboration, "Search for a new hadronic resonance using jet ensembles with CDF”, note 10256, February 2011.

[146] T. Sjostrand, S. Mrenna and P. Skands, "PYTHIA 6.4 physics and manual," JHEP 0605, 026 (2006). [arXiv:hep-ph/0603175].

[147] J. S. Conway, "Pretty Good Simulation of high-energy collisions", 090401 release, http://physics.ucdavis.edu/ conway/research/ software/pgs/pgs4-general.htm

[148] J. Thaler et al, Chameleon version 1.02, July 2006 , http://v1.jthaler.net/olympicswiki/ doku.php?id=lhc_olympics:analysis_tools,

[149] V. M. Abazov et al. [D0 Collaboration], "Measurement of the $t \bar{t}$ production cross section in $p \bar{p}$ collisions at $\sqrt{s}=1.96 \mathrm{TeV}$," Phys. Rev. Lett. 100, 192004 (2008) [arXiv:0803.2779].

T. Aaltonen et al. [CDF Collaboration], "First measurement of the ratio $\sigma_{t \bar{t}} / \sigma_{Z / \gamma^{*} \rightarrow \ell \ell}$ and precise extraction of the $t \bar{t}$ cross section," Phys. Rev. Lett. 105, 012001 (2010) [arXiv:1004.3224]. 


\section{BIBLIOGRAPHY}

[150] S. Chatrchyan et al. [CMS Collaboration], "Measurement of the Top-antitop Production Cross Section in pp Collisions at $\sqrt{s}=7 \mathrm{TeV}$ using the Kinematic Properties of Events with Leptons and Jets," [arXiv:1106.0902].

ATLAS Collaboration, "Measurement of the $t \bar{t}$ production cross-section in $p p$ collisions at $\sqrt{s}=7 \mathrm{TeV}$ using kinematic information of lepton+jets events", note ATLAS-CONF-2011-121, Aug. 2011.

[151] C. T. Hill, “Topcolor: Top quark condensation in a gauge extension of the standard model," Phys. Lett. B266, 419-424 (1991).

[152] L. J. Hall and A. E. Nelson, "Heavy gluons and monojets," Phys. Lett. B 153, 430 (1985).

[153] CDF Collaboration, May, 2011 http://www-cdf.fnal.gov/physics/ewk/2011/wjj/kinematics.html

[154] O. Antunano, J. H. Kuhn and G. Rodrigo, Phys. Rev. D 77, 014003 (2008), [arXiv:0709.1652].

[155] P. H. Frampton, J. Shu and K. Wang, Phys. Lett. B 683, 294 (2010), [arXiv:0911.2955].

[156] M. Bauer, F. Goertz, U. Haisch, T. Pfoh, S. Westhoff, JHEP 1011, 039 (2010), arXiv:1008.0742]. 


\section{BIBLIOGRAPHY}

[157] Y. Bai, J. L. Hewett, J. Kaplan and T. G. Rizzo, JHEP 1103, 003 (2011), arXiv:1101.5203].

[158] A. R. Zerwekh, arXiv:1103.0956].

[159] B. Xiao, Y. k. Wang and S. h. Zhu, [arXiv:1011.0152].

[160] X. P. Wang, Y. K. Wang, B. Xiao, J. Xu and S. H. Zhu, Phys. Rev. D 83, 115010 (2011), [arXiv:1104.1917].

[161] H. Wang, Y. K. Wang, B. Xiao and S. H. Zhu, [arXiv:1107.5769].

[162] J. A. Aguilar-Saavedra and M. Perez-Victoria, [arXiv:1107.2120].

[163] E. Gabrielli, M. Raidal, [arXiv:1106.4553].

[164] R. Barcelo, A. Carmona, M. Masip, J. Santiago, Phys. Rev. D in press [arXiv:1105.3333].

[165] R. Barcelo, A. Carmona, M. Masip, J. Santiago, [arXiv:1106.4054].

[166] E. Alvarez, L. Da Rold, J. I. S. Vietto and A. Szynkman, [arXiv:1107.1473].

[167] R. S. Chivukula, E. H. Simmons and C. P. Yuan, Phys. Rev. D 82, 094009 (2010), [arXiv:1007.0260].

[168] M. I. Gresham, I. W. Kim, K. M. Zurek, Phys. Rev. D83, 114027 (2011), [arXiv:1103.3501]. 


\section{BIBLIOGRAPHY}

[169] G. Tavares, M. Schmaltz [arXiv:1107.0978].

[170] L. J. Hall and A. E. Nelson, Phys. Lett. B 153, 430 (1985), [Journal Server].

[171] P. H. Frampton, S. L. Glashow, Phys. Lett. B190, 157 (1987). [Journal Server].

[172] C. T. Hill, S. J. Parke, Phys. Rev. D49, 4454-4462 (1994), [arXiv:hep-ph/9312324].

[173] C. T. Hill, Phys. Lett. B266, 419-424 (1991), [Journal Server].

[174] P. Ferrario and G. Rodrigo, Phys. Rev. D 78, 094018 (2008), [arXiv:0809.3354].

[175] Q. H. Cao, D. McKeen, J. L. Rosner, G. Shaughnessy, C. E. M. Wagner, Phys. Rev. D81, 114004 (2010), [arXiv:1003.3461].

[176] J. Alwall, M. Herquet, F. Maltoni, O. Mattelaer, T. Stelzer, [arXiv:1106.0522].

[177] N. D. Christensen, C. Duhr, Comput. Phys. Commun. 180, 1614-1641 (2009), [arXiv:0806.4194].

[178] T. Sjostrand, S. Mrenna and P. Skands, JHEP 0605, 026 (2006), [arXiv:0603175].

[179] J. S. Conway, Pretty Good Simulation (PGS).

[180] CDF Public Note, [9913]. 


\section{BIBLIOGRAPHY}

[181] S. Moch, P. Uwer, Phys. Rev. D78, 034003 (2008), [arXiv: 0804 . 1476].

[182] N. Kidonakis and R. Vogt, Phys. Rev. D 78, 074005 (2008), [arXiv: 0805.3844].

[183] M. Cacciari, S. Frixione, M. L. Mangano, P. Nason and G. Ridolfi, JHEP 0809 (2008) 127, arXiv:0804.2800].

[184] T. Aaltonen et al. [CDF Collaboration], Phys. Rev. D 79, 112002 (2009), [arXiv:0812.4036].

[185] V. M. Abazov et al. [D0 Collaboration], Phys. Rev. D 69, 111101 (2004), [arXiv:0308033].

[186] G. Aad et al. [ATLAS Collaboration], New J. Phys. 13, 053044 (2011), [arXiv:1103.3864].

[187] V. Khachatryan et al. [CMS Collaboration], Phys. Rev. Lett. 105, 211801 (2010), [arXiv:1010.0203].

[188] J. Zhu [ATLAS Collaboration], [SUSY'11 Plenary Talk].

[189] B. A. Dobrescu, K. Kong, R. Mahbubani, JHEP 0707, 006 (2007), [arXiv:0703231].

[190] J. Alitti et al. [UA2 Collaboration], Z. Phys. C49, 17-28 (1991), [Journal Server].

[191] T. Aaltonen et al. [CDF Collaboration], [arXiv:1106.4782]. 


\section{BIBLIOGRAPHY}

[192] V. M. Abazov et al. [D0 Collaboration], Phys. Rev. Lett. 101, 221802 (2008), arXiv:0805.3556].

[193] M. A. Doncheski, R. W. Robinett, Phys. Rev. D58, 097702 (1998), [Journal Server].

[194] CDF Collaboration, [W+ Higgs Limits].

[195] D0 Collaboration, [W+ Higgs Limits].

[196] ALEPH Collab., R. Barate et al., Phys. Lett. B495 (2000) 1, [arXiv:0011045].

[197] L3 Collab., M. Acciarri et al., Phys. Lett. B495 (2000) 18, [arXiv: 0011043 ].

[198] OPAL Collab., G. Abbiendi et al., Phys. Lett. B499 (2001) 38, [arXiv: 0101014].

[199] DELPHI Collab., P. Abreu et al., Phys. Lett. B499 (2001) 23, [arXiv:0102036].

[200] J. Abdallah et al. [DELPHI Collaboration], Eur. Phys. J. C 51 (2007) 525, [arXiv:0706.2741].

[201] G. Abbiendi et al. [OPAL Collaboration], Eur. Phys. J. C33, 463-476 (2004), [arXiv:0308067].

[202] P. Achard et al. [L3 Collaboration], Phys. Lett. B586, 151-166 (2004), [arXiv:0402036].

[203] T. Barklow et al. [ALEPH Collaboration], [Note 2001-060 


\section{BIBLIOGRAPHY}

[204] D. E. Kaplan, M. D. Schwartz, Phys. Rev. Lett. 101, 022002 (2008), arXiv:0804.2477].

[205] B. Adeva et al. [L3 Collaboration], Phys. Lett. B263, 551-562 (1991), [Journal Server].

[206] G. Abbiendi et al. [ OPAL Collaboration ], Eur. Phys. J. C20, 601-615 (2001), [arXiv:0101044].

[207] P. Abreu et al. [ DELPHI Collaboration ], Phys. Lett. B456, 322-340 (1999), [Journal Server] .

[208] A. Heister et al. [ALEPH Collaboration], Eur. Phys. J. C27, 1-17 (2003), [Journal Server].

[209] S. Bethke, A. Ricker, P. M. Zerwas, Z. Phys. C49, $59-72$ (1991), [Journal Server].

[210] S. Bethke, Eur. Phys. J. C64, 689-703 (2009), [arXiv:0908.1135].

[211] F. Cuypers, A. F. Falk, P. H. Frampton, Phys. Lett. B259, 173-174 (1991), [Journal Server].

[212] F. Cuypers, P. H. Frampton, Phys. Rev. Lett. 63, 125-127 (1989), [Journal Server].

[213] K. G. Chetyrkin, Acta Phys. Polon. B28, 725-738 (1997), [arXiv: 9610531].

[214] K. Nakamura et al. [ Particle Data Group Collaboration], J. Phys. G G37, 075021 (2010), [PDG Online]. 


\section{Vita}

Gordan Krnjaic graduated Phi Beta Kappa from Reed College in 2005 with a B.A. in Physics. During the 2010-2011 academic year, he was the recipient of a Fermilab Fellowship in Theoretical Physics and spent the year as a visiting student with the FNAL theory group. In September 2012 he will start a postdoctoral fellowship at The Perimeter Institute for Theoretical Physics in Waterloo, Ontario, Canada. 